\title{
SPIN-WAVE SPINTRONICS
}

A Dissertation presented to

the Faculty of the Graduate School

at the University of Missouri-Columbia

In Partial Fulfillment

of the Requirements for the Degree

Doctor of Philosophy

by

TIANYU LIU

Dr. Giovanni Vignale, Dissertation Supervisor

DEC 2013 
(C) Copyright by Tianyu Liu 2013

All Rights Reserved 
The undersigned, appointed by the dean of the Graduate School, have examined the dissertation entitled

\section{SPIN-WAVE SPINTRONICS}

presented by Tianyu Liu,

a candidate for the degree of doctor of philosophy, and hereby certify that, in their opinion, it is worthy of acceptance.
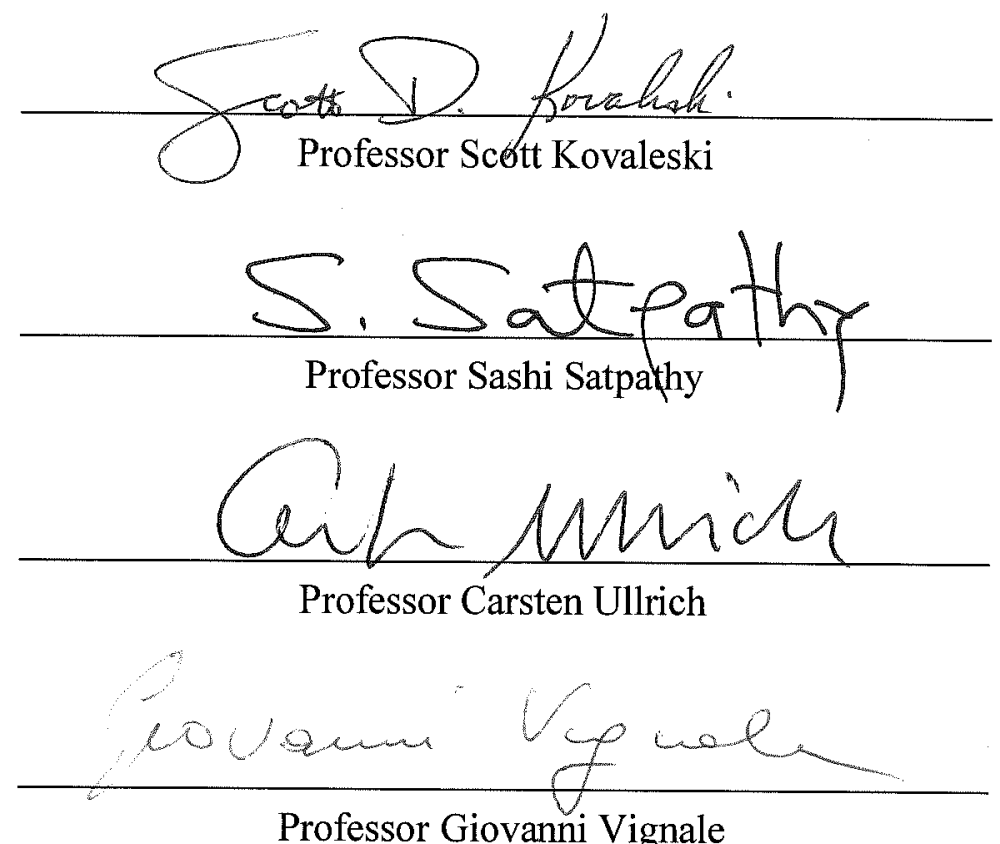

Professor Giovanni Vignale 


\section{ACKNOWLEDGMENTS}

I would like to express the deepest appreciation to my advisor, Prof. Giovanni Vignale. Dr. Vignale is clearly an outstanding physicist, a great teacher, a productive writer, but I recognize him first as an extraordinary mentor. From him, I have acquired not only the knowledge but also the philosophy of being a professional in the

academic world. He has served as an excellent role model to future faculty members like me.

I would like to thank Prof. Michael Flatté for bringing up the idea of magnon drag and for the fruitful discussions with him on this topic. I also want to thank to ARO Grant No. W911NF-08-1-0317 for the financial support through my doctoral program. 


\section{Contents}

ACKNOWLEDGMENTS ................ ii

LIST OF FIGURES $\ldots \ldots \ldots \ldots \ldots \ldots$ vi

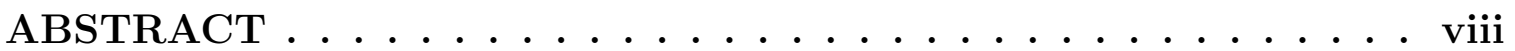
CHAPTER

1 Introduction ..................... 1

2 Dzyaloshinskii-Moriya Interaction - Microscopic Perspective . . . 7

2.1 Heisenberg Hamiltonian Modified by Spin-Orbit Coupling . . . . . . . 8

2.2 Effective Spin-Orbit Coupling Coefficient . . . . . . . . . . . . 12

2.2.1 Electron Wave Function on Magnetic Ions . . . . . . . . . . 12

2.2.2 Electron Wave Function in Superexchange Model . . . . . . . 14

2.2 .3 DM Interaction $\ldots \ldots \ldots \ldots \ldots \ldots$

3 Electric Control of Phase Shift in Thin Films f . . . . . . . 18

3.1 Model and equation of motion . . . . . . . . . . . . . . . 19

3.2 Dispersion of Transverse Spin Waves in a magnetic film . . . . . . 23

3.3 Electric-field induced phase shift . . . . . . . . . . . . . . . 29

4 Spin-Wave Interferometer: Voltage-Controlled NOT Gate . . . . . 32

4.0.1 Spin wave dispersion $\ldots \ldots \ldots \ldots \ldots \ldots$

4.0 .2 Spin wave interferometer . . . . . . . . . . . 36

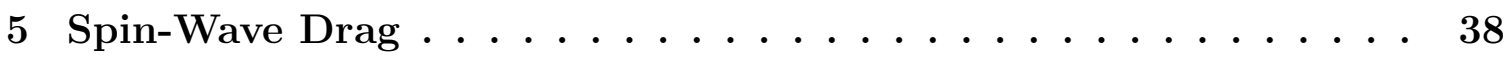


5.1 Quantization of Ferromagnetic Spin Waves . . . . . . . . . . . . 40

5.1.1 Heisenberg Hamiltonian for Spin-1/2 . . . . . . . . . . . . . 41

5.1.2 Second Quantization ................ 43

5.2 Thermodynamic Definitions for magnon current density and heat current density . . . . . . . . . . . . . . . . . . 45

5.3 Drag Resistivities . . . . . . . . . . . . . . . . . 47

5.4 Rate of Change of Momentum . . . . . . . . . . . . . . . 54

5.5 Rate of Change of Thermal Current . . . . . . . . . . . . . 61

5.6 Temperature-Dependence of The Drag Resistivities . . . . . . . . . 62

5.6.1 For fixed chemical potential . . . . . . . . . . . . 63

5.6.2 For fixed number of pumped magnons . . . . . . . . . . . 70

5.7 Distance Dependence of the drag resistivities . . . . . . . . . . . . 72

5.8 Estimation for Measurable Quantities . . . . . . . . . . . . . . 72

5.9 Conclusion . . . . . . . . . . . . . . . . . 75

\section{APPENDIX}

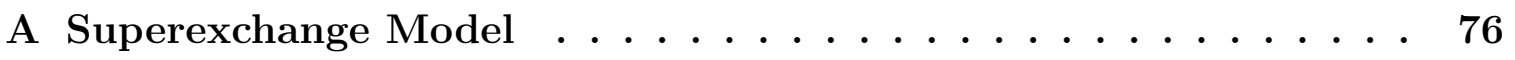

A.1 Hamiltonian . . . . . . . . . . . . . . . . 76

A.2 The Van Vleck Transformation . . . . . . . . . . . . . 78

A.3 Effective matrix for states of interest . . . . . . . . . . . . 79

B Magnon-Magnon Interaction ............... 82

B.1 "Holstein-Primakoff" Transformation . . . . . . . . . . . . . 83

B.2 Bogoliubov Transformation . . . . . . . . . . . . . . . . . 90 
B.3 Rate of Change of Momentum and Thermal Current - Four-Magnon

Interaction . . . . . . . . . . . . . . . . . . . 93

C Magnon Response Functions $\ldots \ldots \ldots \ldots$

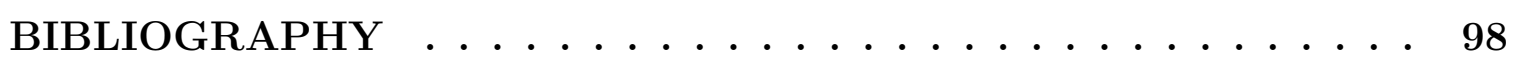

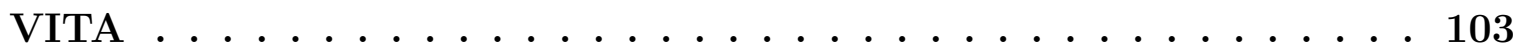




\section{List of Figures}

Figure

Page

2.1 Superexchange model: two half-filled magnetic ions connected by an

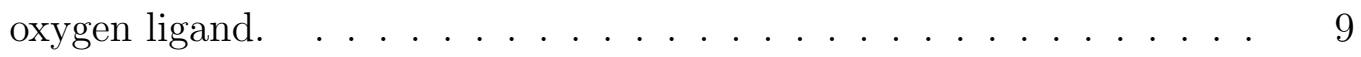

3.1 Schematic illustration of a tangentially magnetized film. . . . . . 19

3.2 Comparison of the dispersion obtained from perturbation theory and numerical diagonalization. . . . . . . . . . . . . . . 26

3.3 Dispersion of spin waves propagating in a tangentially magnetized film in the absence of electric fields. . . . . . . . . . . . . . 28

3.4 Dispersion of spin waves propagating in a tangentially magnetized film in the presence of electric fields. . . . . . . . . . . . 28

3.5 Wave vector as a function of electric field. . . . . . . . . . . . 29

3.6 Shift of wave vector by an electric field as a function of $k \ldots \ldots$. . 30

3.7 Sketch of possible experimental set up for testing the effect of the electric field on the group velocity of spin waves. . . . . . . . . .

4.1 A Mach-Zehnder spin-wave interferometer in the presence of radial $\mathbf{E}$ field. . . . . . . . . . . . . . . . . . . 33 
4.2 Dispersion and transmission probability of spin waves in the ferromagnetic ring. . . . . . . . . . . . . . . . . . 36

5.1 Schematic illustration of spin-wave drag. . . . . . . . . . . . . 41

5.2 A single local spin-flip excitation of a ferromagnetic system. . . . . . 42

5.3 Interlayer three-magnon interactions. . . . . . . . . . . . . 50

5.4 Interlayer four-magnon interactions. The figure shows the outgoing processes of magnons in layer 1: 1 and 2 label the different layers; A and B correspond to the interaction amplitudes $W(\mathbf{k})$ and $W(\mathbf{p})$, respectively; (f) illustrates the Coulomb-like interaction where $W_{q}$ emphasizes that the transition amplitude depends on the momentum transfer only. 51

5.5 Relative direction of $\mathbf{P}_{i}$ and $\mathbf{k} . \hat{\boldsymbol{\zeta}}$ is along $-\mathbf{M}_{s} \ldots \ldots \ldots \ldots 2$

5.6 Relative direction of induced fields and driving current. . . . . . . 55

5.7 The matrix elements of $\tilde{\mathbf{C}}^{12}$ as a function of $T \ldots \ldots \ldots \ldots$

5.8 Comparison of the drag resistivities as a function of $T$ for fixed $\mu_{2}$ and those for fixed number of pumped magnons. . . . . . . . . . . 71

5.9 The power law of the drag resistivity with respect to $d . \quad \ldots \ldots 72$

A.1 Subspace $S_{z}^{\text {total }}=0 . \quad \ldots \ldots \ldots \ldots \ldots \ldots \ldots \ldots \ldots \ldots$

A.2 Subspace $S_{z}^{\text {total }}= \pm 1 \ldots \ldots \ldots \ldots \ldots \ldots \ldots \ldots \ldots \ldots \ldots \ldots \ldots \ldots \ldots$

B.1 Two right-hand reference frame. . . . . . . . . 85

B.2 Schematic picture of ferromagnetic bilayer system. . . . . . . 87 


\begin{abstract}
Spin waves in insulating magnets are ideal carriers for spin currents with low energy dissipation. An electric field can modify the dispersion of spin waves, by directly affecting, via spin-orbit coupling, the electrons that mediate the interaction between magnetic ions. Our microscopic calculations based on the superexchange model indicate that this effect of the electric field is sufficiently large to be used to effectively control spin-wave spin currents. We apply these findings to the design of spin-wave phase shifter, and a spin-wave interferometric device, which acts as a logic inverter and can be used as a building block for room-temperature, low-dissipation logic circuits. This part of work has been published in Phys. Rev. Lett. and J. Appl. Phys..

Besides the magneto-electric effect, we also study the magneto-thermal effect that couples the spin-wave spin current to the thermal current. In analogy to Coulombdrag effect, we propose a spin-wave drag effect due to magnetic dipolar interaction in a ferromagnetic bilayer system. Compared with Coulomb drag effect in electron gas bilayer, we find that here the interlayer transport coefficients abnormally increase as the temperature decreases because of bosonic statistics of magnons. Besides, the coefficients show an angular dependence on the angle between saturation magnetization and spin-wave spin current.
\end{abstract}




\section{Chapter 1}

\section{Introduction}

Spin waves - the collective oscillations of the magnetization in magnetic materials have attracted attention in recent years as carriers of spin information for magnetoelectronic devices. It opens new possibilities for spintronics - the spin-wave spin-

tronics/magnonics $[1,2]$. In conventional spintronics, the spin current is carried by mobile conduction electrons/holes, which inevitably dissipate energy as they move. In contrast, the spin current in spin-wave spintronics is carried by a spin wave with no charge displaced. This makes magnonic devices consume much less energy than spintronic devices where much of the energy is dissipated through Joule heating. There are some other advantages of magnonics, such as it works at the frequency from $\mathrm{GHz}$ to $\mathrm{THz}$ at room temperature. And because spin waves with different frequencies do not interfere with each other, one can accomplish majority gates in magnonic circuitry without being constrained by the circuit complexity.

The spin-wave spin current propagating in insulating materials such as yttrium iron garnet $\left(\mathrm{Y}_{3} \mathrm{Fe}_{5} \mathrm{O}_{12}, \mathrm{YIG}\right)$ is of particular interest [3]: It is totally free of energy 
dissipation from Joule heating, and almost free of dissipation from other sources (e.g. electron-magnon scattering). Recently, injection and extraction of spin-wavecarried spin currents in a YIG waveguide have been demonstrated by several different methods (spin transfer torque [3], parametric pumping [4], spin Hall effect [3]) and various ideas for making analog magnonic circuits [1] and digital magnonic gates [5] have been explored. A common feature of all these proposals is that they presuppose the ability to control the frequency and/or the wave vector of the spin waves. For example, in a NOT logic gate one needs to control the wave vector of a spin wave so as to drive the output of a spin wave interferometer from large (logical 1) to small (logical 0). The traditional way to accomplish this is through the application of magnetic fields via currents in wires. However, the magnetic fields generated in this manner are quite extended and not suitable for the local control of a nanoscale device. Electric fields, on the other hand, can be applied locally through gates and thus offer better possibilities for nano-electronics.

The possibility of using electric fields to control spin waves has been known for decades. The first observation of electric-field-induced frequency shift of spin waves in Lithium ferrite dates back to the late 1970's [6]. However, those shifts were found to be very small $(0.01 \%)$ as they relied on minute changes in the magneto-crystalline anisotropy energy. In recent years, multiferroic materials have emerged as better candidates to accomplish electric control of spin waves. In these materials an electric polarization $(\mathbf{P})$, either spontaneous [7] or induced by a magnetic field or magnetic impurities [8], coexists with and is coupled to spontaneous magnetic moments. The electric polarization can be driven by an electric field around a large hysteresis loop, and the resulting variation of the spin wave frequency can be as large as 30\% in a 
material like $\mathrm{BiFeO}_{3}(\mathrm{BFO})$ at room temperature [9].

Unfortunately, the most popular spin wave material - YIG, whose coherence length approaches the centimeter scale - is not a good candidate for this form of magneto-electric control, since it does not have a spontaneous electric polarization [10]. This does not mean, however, that magneto-electric effects are absent. In chapters 2, 3 and 4, we will show that an electric-field induced phase shift could be realized in YIG through Dzyaloshinskii-Moriya interaction.

Our study of spin waves begins in Chapter 2, where we derive the Heisenberg Hamiltonian $\left(J_{e x} \mathbf{S}_{1} \cdot \mathbf{S}_{2}\right)$ from a microscopic model for superexchange interaction. Such interaction is crucial to form the magnetic order in YIG, the most popular material in magnonics. When including in the microscopic model the spin-orbit (SO) coupling between electrons and the applied electric field (E), we find that the real exchange coefficient $J_{e x}$ becomes complex and its imaginary part yields the DzyaloshinskiiMoriya (DM) interaction that is linear in $\mathbf{E}$. Intuitively, the DM interaction is thought to be very weak as the SO coupling is treated as a relativistic effect. Yet, our principal result in this chapter shows that the strength of SO coupling in materials having $3 d$ ions (such as YIG) is orders of magnitude larger than that in vacuum (as was used in Ref. [11]).

In Chapter 3, we examine the impact of the DM term on spin waves that can be realistically propagated in a thin film wave guide, such as the one considered in the experiments of Ref. [4]. Such waves can be qualitatively described as magnetostatic waves (at wavelengths longer than $1 \mu \mathrm{m}$, when the magnetostatic long-range interactions dominate) and exchange waves (at wavelengths shorter than $0.1 \mu \mathrm{m}$, when the short-range exchange interaction dominates). Notice that in both cases the wave- 
length is large enough to justify the use of the continuum approximation. We focus on the lowest lying mode of the wave guide, such that the equilibrium magnetization lies in the plane of the film. For this geometry, the largest effect, as measured by the relative wave vector shift $\delta k / k$, is found when the wave vector of the spin wave is perpendicular to the magnetization. The effect declines slowly as $k$ increases and eventually enters saturation in the range of exchange waves. We are now collaborating with Prof. Tang's group at Yale University to measure the electric-field-induced phase shift in a YIG waveguide with spin waves excited by microwave antennas and observe a linear increase of the phase shift with increasing the frequency of the spin waves for a fixed electric field. Such an observation is in agreement with our theory since the spin waves excited by antennas is in the magnetostatic range where $\delta k$ is approximately linear in $k$. Further measurements towards proving the existence of DM interaction in YIG are in progress.

An attractive feature of magnonics is that one could encode information not only in the phase of spin waves but also in the amplitude of spin waves. Kostylev et al. [5], have designed an ingenious scheme of spin-wave logic, based on the interference between spin waves traveling along different arms of a Mach-Zehnder interferometer (a schematic illustration of a Mach-Zehnder spin wave interferometer is shown in Fig. 4.1). In their design, the logic output depends on the amplitude of the spin waves after interference: the constructive interference corresponding to logic "1", and the destructive interference corresponding to logic "0". However, the knob controlling the interference is a current threading the ring, which inevitably consumes energy through Joule heating. In Chapter 4, we study the spin waves propagating in the same ring interferometer, but in the presence of a radial electric field, so that we can 
accomplish a voltage-controlled NOT gate by virtue of the DM interaction.

With the development of spintronics, people discovered the spin-dependent Seebeck effect [12] and spin-dependent Peltier effect [13] in 2010 and 2012, respectively. Ordinary Seebeck effect is an thermal-electric effect that converts a temperature difference directly to electricity. It has been widely used in making thermocouples. Conversely, an isothermal electric current is accompanied by a thermal current, which is called the Peltier effect. These two effects together construct the complete relations between electric current and thermal current. The spin-dependent Seebeck/Peltier effect appears when the electric current is spin-polarized. These spin-dependent thermoelectric effects lead to a new field of spintronics - caloritronics [14], where thermal currents are used to inject spin currents into normal metals. The basics of caloritronics is the spin accumulation at a ferromagnetic/non-magnetic metal (FM/NM) interface, which is created by running a thermal current across the interface.

Spin Seebeck effect $[15,16,17]$ is different from spin-dependent Seebeck effect since it does not rely on the spin diffusion length. More surprisingly, it can be found even in an insulator [17]. In contrast to spin-dependent Seebeck effect, it is the spin-wave spin current that couples to the thermal current in spin Seebeck effect. Since it involves magnetic moments instead of electric charges, we call it magnetothermal effect. Although the first experiment on spin Seebeck effect was done in 2008, there still lacks a prevalently adopted theory in this area. One of the barriers is the rich multitude interactions among electrons, magnons and phonons. Notice that the thickness of the films used in these spin Seebeck effect experiments is of the order of micrometer; and cutting off the material does not affect the results. These two points indicates that the magnetic dipolar interaction should play a key role in spin 
Seebeck effect because of its long-ranged characteristic. We, therefore, propose a spinwave drag measurement in an insulating ferromagnetic bilayer system to separate the magnetic dipolar interaction from the other interactions, see Chapter 5.

Chapter 5 departs from the previous semi-classical treatment of spin waves: It describes spin waves in the language of second quantization. Like phonons are the quanta of lattice oscillations, magnons are the quanta of magnetization oscillations. Thus, spin-wave spintronics is also called magnonics. While studying the electricfield-induced phase shift in thin films, we found that the magnetic dipolar interaction under magnetostatic approximation has the form similar to Coulomb interaction. This inspired us to use the spin-wave drag effect in ferromagnetic bilayer to study the magnetic dipolar interaction. In Coulomb drag measurement, a steady current in the active layer produces a voltage difference in the passive layer; here, we propose a spin-wave drag measurement, where two ferromagnetic films are thermally isolated and well separated - only magnetic dipolar interaction exists between the two layers. We keep the active layer at a homogeneous temperature, and launch a steady spinwave spin current in it. As a result of interlayer dipolar interaction, a temperature gradient and a chemical potential gradient are expected to be induced in the passive layer. The results show that the drag effect is increased with decreasing temperature and tends to zero when the temperature drops below the energy gap of the magnon dispersion. Unlike ordinary Coulomb drag, which is isotropic, magnon drag strongly depends on the angle between the magnon current and the saturation magnetization, and achieves maximum (minimum) when the two are parallel (perpendicular).

Throughout the dissertation, unless otherwise noted, I use the SI units. 


\section{Chapter 2}

\section{Dzyaloshinskii-Moriya Interaction - Microscopic Perspective}

The ferromagnet we are interested in is YIG, whose magnetic order mainly arises from the superexchange interaction between $\mathrm{Fe}^{3+}$ in octahedral (a) sites and tetrahedral (d) sites. The fact that the numbers of (a) and (d) sites per unit cell are different makes YIG a ferrimagnet. However, the long wave-length spin waves, whose energy is less than about $40 \mathrm{~K}$, can be understood with an effective ferromagnetic exchange coupling between "block spins" $S_{i}$, one per unit cell [18].

YIG is an insulator, which allows us to use tight-binding approximation to describe its electronic structure. Noting that $F e^{3+}$ has half-filled $3 d$ out shell, we begin with the two sites superexchange model shown in Fig. 2.1. With this simplified model, we can focus on the physical essence of the effect of spin-orbit (SO) coupling on Heisenberg Hamiltonian (see Eq.2.6). In Sec. 2.2, we refine our model by taking into account the coupling between electron spins and $p$ - $d$ hybrid orbitals (i.e. the intrinsic 
SO coupling) in obtaining the wave functions of the electrons that contributes to the formation of local electric dipole. Further, we achieve at the effective SO coupling coefficient attributed to the external electric field in terms of microscopic parameters, such as electron hopping coefficient, distance between neighboring magnetic sites, etc., as shown in Eq. 2.20.

\subsection{Heisenberg Hamiltonian Modified by Spin-Orbit Coupling}

We start from the superexchange model shown in Fig. 2.1, which can be described by the following Hamiltonian

$$
\begin{aligned}
& H_{\text {super }}=H_{0}+H_{t}+H_{U}, \\
& H_{0}=\epsilon_{0} \sum_{\sigma} c_{0 \sigma}^{\dagger} c_{0 \sigma}+\epsilon_{1} \sum_{i=1}^{2} \sum_{\sigma} c_{i \sigma}^{\dagger} c_{i \sigma}, \\
& H_{t}=-t \sum_{\sigma}\left(c_{1 \sigma}^{\dagger} c_{0 \sigma}+c_{2 \sigma}^{\dagger} c_{0 \sigma}+\text { h.c. }\right), \\
& H_{U}=U \sum_{i=1}^{2} \sum_{\sigma} c_{i \sigma}^{\dagger} c_{i \sigma} c_{i \bar{\sigma}}^{\dagger} c_{i \bar{\sigma}},
\end{aligned}
$$

where $c\left(c^{\dagger}\right)$ is the creation (annihilation) operator of ligand electrons, which can hop forth and back only between oxygen ligand and the metal ions, $\epsilon_{1}$ and $\epsilon_{0}$ are the orbital energies of a metal ion and the oxygen ligand, respectively. The large repulsion energy $U(\sim 8 \mathrm{eV})$ between two electrons on the same metal ion allows for a maximum occupancy of two electrons per ion (the repulsion between the electrons in the oxygen ligand is negligible in comparison). 


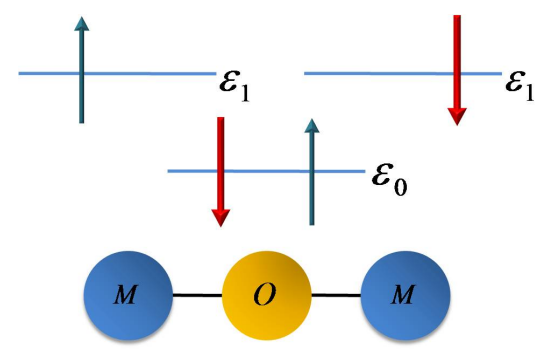

Figure 2.1: Superexchange model: two half-filled magnetic ions connected by an oxygen ligand.

The fact $[18,19]$ that $t(\simeq 0.8 \mathrm{eV})$ is much smaller that $U$ allows us to use perturbation theory. Keeping up to the fourth order of $t$ yields the effective interaction between the spins on the magnetic ions (see Appendix A):

$$
H_{e f f} \simeq\left(-\frac{2 t^{2}}{V}+\frac{3 t^{4}}{V^{3}}-\frac{t^{4}}{V^{2} U}\right) \mathbf{1}+\left(\frac{4 t^{4}}{V^{2} U}+\frac{4 t^{4}}{V^{3}}\right)\left[\frac{1}{2}\left(S_{1}^{+} S_{2}^{-}+\text {h.c. }\right)+S_{1}^{z} S_{2}^{z}\right]
$$

where $V=\epsilon_{1}-\epsilon_{0}+U$ is the energy difference between the state with oxygen doubly occupied and the state with a metal site doubly occupied, and the zero energy point has been chosen to be $\epsilon_{0}+\epsilon_{1}$. Setting $J_{e x}=\frac{4 t^{4}}{V^{2} U}+\frac{4 t^{4}}{V^{3}} \approx \frac{8 t^{4}}{V^{3}}$ and dropping the constant term, we obtain the Heisenberg interaction

$$
H_{H}=J_{e x} \mathbf{S}_{1} \cdot \mathbf{S}_{2} .
$$

A positive $J_{e x}$ implies that the interaction between neighboring magnetic ions is antiferromagnetic. However, this antiferromagnetic interaction gives rise to a ferromagnetic interaction between "block spins" in YIG, due to the unequal magnitudes of the anti-parallel magnetic moments in each block.

We now augment the usual superexchange model by the inclusion of a spin-orbit 
(SO) interaction of the form

$$
H_{S O}=-\frac{\hbar}{2 m E_{S O}}(\mathbf{p} \times e \mathbf{E}) \cdot \boldsymbol{\sigma}
$$

where $E_{S O}$ is an energy scale associated with the inverse of the strength of SO coupling (i.e., $E_{S O}$ is large when $\mathrm{SO}$ coupling is weak and vice versa), $e$ is the elementary charge, and $\boldsymbol{\sigma}$ is the Pauli matrix. For electrons in vacuum $E_{S O}$ is of the order of the electron rest energy $m c^{2} \sim 5 \times 10^{5} \mathrm{eV}$, but we will see in Sec. 2.2, in a magnet with $3 d$ magnetic ions, the value of $E_{S O}$ is orders of magnitudes smaller $(\sim 1 \mathrm{eV})$, which corresponds to a very strong SO coupling.

It is easy to see that the inclusion of this interaction is equivalent to the inclusion of a spin-dependent vector potential $\mathbf{A}=\frac{\hbar}{2 E_{S O}} \mathbf{E} \times \boldsymbol{\sigma}$, which in turn modifies the hopping term $H_{t}$ by a spin-dependent phase factor $\exp \left[\frac{e a}{4 E_{S O}}(\mathbf{E} \times \boldsymbol{\sigma}) \cdot \hat{\mathbf{e}}_{i j}\right]$, where $\hat{\mathbf{e}}_{i j}$ is the unit vector connecting neighboring magnetic ions, that is

$$
H_{t}=-t \sum_{\sigma}\left(c_{1 \sigma}^{\dagger} c_{0 \sigma} e^{-i \alpha \sigma}+c_{2 \sigma}^{\dagger} c_{0 \sigma} e^{i \alpha \sigma}+\text { h.c. }\right)
$$

where $\alpha=\frac{e a E}{4 E_{S O}}$, provided that the external electric field, the motion of the electron, and the electron spin $(\sigma)$ are perpendicular to each other. Notice that the phase $\alpha$ is proportional to $a$, the distance between neighboring magnetic sites, and independent of the direction of the local magnetic moments: one can therefore switch to the "block spins" description by simply reinterpreting $a$ as the distance between neighboring 
blocks. The resulting spin Hamiltonian takes the form

$$
\begin{aligned}
H & =-J_{e x}^{\prime} \sum_{<i, j>} S_{i}^{z} S_{j}^{z}+\frac{1}{2}\left(e^{i 2 \alpha_{i j}} S_{i}^{+} S_{j}^{-}+e^{-i 2 \alpha_{i j}} S_{i}^{-} S_{j}^{+}\right) \\
& \simeq-J_{e x}^{\prime} \sum_{<i, j>}\left\{\left(\mathbf{S}_{i} \cdot \mathbf{S}_{j}\right)+\sin 2 \alpha_{i j}\left(\mathbf{S}_{i} \times \mathbf{S}_{j}\right)_{z}\right\}
\end{aligned}
$$

where $\alpha_{i j} \equiv 2 \alpha(i-j),-J_{e x}^{\prime}$ is the effective exchange coupling for the spin blocks and $z$ is in the direction perpendicular to $\mathbf{E}$ and $\hat{\mathbf{e}}_{i j}$. In the last line of Eq. (2.6), we obtain, in addition to the normal Heisenberg Hamiltonian (the first term), a DM interaction [20],

$$
H_{D M}=\sum_{<i, j>} \mathbf{D}_{i j} \cdot\left(\mathbf{S}_{i} \times \mathbf{S}_{j}\right) \quad \text { with } \quad \mathbf{D}_{i j}=-J_{e x}^{\prime} \frac{e a}{E_{S O}} \mathbf{E} \times \hat{\mathbf{e}}_{i j}
$$

whose strength is linear in $E$. An electric-field induced anisotropy is also present, but is an effect of order $E^{2}$ and has therefore been neglected for weak electric field.

In spite of the presence of the noncollinear DM term, the ferromagnetic configuration is still the ground state of (2.6). To show this, we make $\mathbf{S}_{i}=\mathbf{S}_{0}+\delta \mathbf{S}_{i}$, where $\delta \mathbf{S}_{i}$ is small deviation perpendicular to $\mathbf{S}_{0}$. Then the variation of the DM term up to the second order of $\delta \mathbf{S}_{i}$ is

$$
\delta H_{D M} \approx \sum_{<i, j>} \mathbf{D}_{i j} \cdot\left(\delta \mathbf{S}_{i} \times \mathbf{S}_{0}+\delta \mathbf{S}_{i} \times \delta \mathbf{S}_{j}\right)
$$

where $\mathbf{D}_{i j}$ is defined by Eq.(2.7). Provided the magnet having inversion symmetry $\mathbf{e}_{i j}=-\mathbf{e}_{j i}$, we see that $\sum_{j} \mathbf{D}_{i j}=0$, which means $\delta H_{D M}=0$ up to the first order of $\delta \mathbf{S}_{i}$. Hence, the ground state is still ferromagnetic. However, the DM term will definitely modify the spin-wave frequency, which involves a correction to the ground 
state energy at the second order in $\delta \mathbf{S}_{i}$. Further, we can clearly see that it is only the component of the $\mathbf{D}$ parallel to $\delta \mathbf{S}_{i} \times \delta \mathbf{S}_{j}$ (i.e. to the direction of the equilibrium magnetization) that plays a role in the modification.

\subsection{Effective Spin-Orbit Coupling Coefficient}

In order to quantify the effect of an applied electric field on the propagation of spin waves, we have to know the magnitude of $E_{S O}$. This turns out to be the most tedious part in this work. Notice that we have suppose the hopping coefficient $t$ to be real in Eq. (2.5). It indicates that the phase factor induced by the $\mathrm{SO}$ coupling is not only from the external electric field but also from the internal orbital field.

We will still work on the two-site model as shown in Fig. 2.1 in this section, but refine our model to exclude the contribution from internal SO coupling by following the idea of Ref. [7]. We first calculate the electron wave functions in the presence of internal spin-orbit coupling $\mathbf{L} \cdot \mathbf{S}$, then obtain the local electric dipole, whose interacting with the external electric field gives us the DM interaction. Comparing with the DM interaction obtained in Sec. 2.1, we finally achieve at $E_{S O}$ expressed in terms of microscopic parameters, such as electron hopping coefficient, distance between neighboring magnetic sites, etc., as shown in Eq. 2.20. By substituting the parameters of YIG $[18,19], E_{S O}$ is expected to be $3 \mathrm{eV}$.

\subsubsection{Electron Wave Function on Magnetic Ions}

We start with the orbital wave functions of $\mathrm{Fe}^{3+}$ in the presence of octahedral ligand field according to the crystal structure of YIG [18]. It is well known that the $t_{2 g}$ 
orbitals, i.e., $d_{x y}, d_{y z}$ and $d_{z x}$ have energy lower that the $e_{g}$ orbitals in such a ligand field. Considering the internal SO coupling $\lambda \mathbf{L} \cdot \mathbf{S}$ as a perturbation, where $\lambda$ is negative due to $\mathrm{Fe}^{2+}$ is more than half-filled, we obtain the two-fold degenerate states with the lowest energy by applying the degenerate perturbation theory to the second order:

$$
\begin{aligned}
|a\rangle & =\frac{1}{\sqrt{3}}\left(\left|d_{x y}, \uparrow\right\rangle+\left|d_{y z}, \downarrow\right\rangle+i\left|d_{z x}, \downarrow\right\rangle\right), \\
|b\rangle & =\frac{1}{\sqrt{3}}\left(\left|d_{x y}, \downarrow\right\rangle-\left|d_{y z}, \uparrow\right\rangle+i\left|d_{z x}, \uparrow\right\rangle\right),
\end{aligned}
$$

where the electron spin is supposed to be quantized along $z$ axis.

Since the out shell of $\mathrm{Fe}^{3+}$ is half-filled, the virtual hopping electrons provided by the ligand oxygen should be repulsed by the local magnetic moment of the metal sites. (It is not real hopping because YIG is an insulator.) Therefore, we consider the Hamiltonian: $H_{U}=U \boldsymbol{\sigma} \cdot \mathbf{n}$, where $U$ is the repulsive energy, $\boldsymbol{\sigma}$ is the Pauli matrix of electron spin, and $\mathbf{n}=(\cos \phi \sin \theta, \sin \phi \sin \theta, \cos \theta)$ is the direction of the magnetic moment on a metal site. Write $H_{U}$ in the basis of $\{|a\rangle,|b\rangle\}$,

$$
H_{U}=\frac{U}{3}\left(\begin{array}{cc}
-\cos \theta & \sin \theta e^{-i \phi} \\
\sin \theta e^{i \phi} & \cos \theta
\end{array}\right)
$$

Diagonalizing $H_{U}$ gives us two states

$$
\begin{aligned}
|A P\rangle & =\cos \frac{\theta}{2}|a\rangle-e^{i \phi} \sin \frac{\theta}{2}|b\rangle, \\
|P\rangle & =\sin \frac{\theta}{2}|a\rangle+e^{i \phi} \cos \frac{\theta}{2}|b\rangle,
\end{aligned}
$$


with eigen energies $-\frac{U}{3}$ and $\frac{U}{3}$, respectively. Therefore, the virtual hopping electron is in its ground state when its spin antiparallel to the magnetic moment. It is useful to write the state $|A P\rangle$ in terms of $d$ orbitals explicitly for doing the hybridization with the oxygen's $p$ orbitals later.

$$
|A P\rangle=\sum_{\sigma} A^{x y, \sigma}\left|d_{x y}, \sigma\right\rangle+A^{y z, \sigma}\left|d_{y z}, \sigma\right\rangle+A^{z x, \sigma}\left|d_{z x}, \sigma\right\rangle
$$

where

$$
\begin{aligned}
A^{x y, \uparrow} & =\frac{1}{\sqrt{3}} \cos \frac{\theta}{2}, \quad A^{x y, \downarrow}=-\frac{1}{\sqrt{3}} \sin \frac{\theta}{2} e^{i \phi}, \\
A^{y z, \uparrow} & =\frac{1}{\sqrt{3}} \sin \frac{\theta}{2} e^{i \phi}, \quad A^{y z, \downarrow}=\frac{1}{\sqrt{3}} \cos \frac{\theta}{2}, \\
A^{z x, \uparrow} & =-\frac{i}{\sqrt{3}} \sin \frac{\theta}{2} e^{i \phi}, \quad A^{z x, \downarrow}=\frac{i}{\sqrt{3}} \cos \frac{\theta}{2} .
\end{aligned}
$$

\subsubsection{Electron Wave Function in Superexchange Model}

Now consider the two-site model in Fig. 2.1. The total Hamiltonian still has the form of $H_{\text {supper }}=H_{t}+\sum_{i} H_{U i}(i=1,2)$, the same as Eq. (2.1) in the sense that $H_{0}$ is set to be zero in getting the effective Heisenberg Hamiltonian (2.3). On the other hand, they are different considering that now we have known the eigen states for $H_{U i}$ and that $H_{t}$ is written in terms of $p$ and $d$ orbitals, i.e.,

$$
H_{t}=t \sum_{\sigma}\left(p_{y, \sigma}^{\dagger} d_{x y, \sigma}^{(1)}+p_{z, \sigma}^{\dagger} d_{z x, \sigma}^{(1)}+\text { h.c. }\right)-t \sum_{\sigma}\left(p_{y, \sigma}^{\dagger} d_{x y, \sigma}^{(2)}+p_{z, \sigma}^{\dagger} d_{z x, \sigma}^{(2)}+\text { h.c. }\right)
$$

where $t$ is again the hopping coefficient. Notice that $t$ is actually the overlap integral between $\left|p_{\alpha}\right\rangle$ and $\left|d_{\beta}^{(i)}\right\rangle$, where $\alpha=x, y, z ; \beta=x y, y z, z x$; and $i=1,2$ 
denotes the two metal sites. As a result, hops from $\mathrm{M} 1$ to $\mathrm{O}$ and from M2 to $\mathrm{O}$ change signs, and some hops are even forbidden (for example, $\left\langle p_{x}\left|\nabla^{2}\right| d_{y z}\right\rangle=0$ ). Now expand the total Hamiltonian in the eight-dimensional space spanned by the bases $\left\{|A P\rangle_{i},|P\rangle_{i},\left|p_{\alpha}, \sigma\right\rangle\right\}$ and treat the hopping term as a perturbation. We obtain the two lowest states wave functions

$$
\begin{aligned}
\left|\psi_{ \pm}\right\rangle= & \pm \frac{1}{\sqrt{2}} \frac{e^{-i \frac{\delta \phi}{2}} \Theta}{|\Theta|}\left[|A P\rangle_{1}-\frac{t}{V} \sum_{\sigma}\left(A_{(1)}^{x y, \sigma}\left|p_{y}, \sigma\right\rangle+A_{(1)}^{z x, \sigma}\left|p_{z}, \sigma\right\rangle\right)\right] \\
& +\frac{1}{\sqrt{2}}\left[|A P\rangle_{2}+\frac{t}{V} \sum_{\sigma}\left(A_{(2)}^{x y, \sigma}\left|p_{y}, \sigma\right\rangle+A_{(2)}^{z x, \sigma}\left|p_{z}, \sigma\right\rangle\right)\right],
\end{aligned}
$$

where $\delta \phi=\phi_{1}-\phi_{2}$ and $\Theta=\cos \frac{\theta_{1}}{2} \cos \frac{\theta_{2}}{2} e^{-i \delta \phi / 2}+\sin \frac{\theta_{1}}{2} \sin \frac{\theta_{2}}{2} e^{i \delta \phi / 2}$. The corresponding eigen energies are $E_{ \pm}=-\frac{2}{3} \frac{t^{2}}{V}(1-|\Theta|)$. Again, $V$ is the energy difference between the states with oxygen doubly occupied and the states with one metal site doubly occupied. The ligand oxygen provides two hopping electrons, therefore, the twoelectron wave function is

$$
\left.|\psi\rangle=\frac{1}{\sqrt{2}}\left[\left|\psi_{+}\left(\mathbf{r}_{1}\right)\right\rangle\left|\psi_{-}\left(\mathbf{r}_{2}\right)-\right| \psi_{+}\left(\mathbf{r}_{2}\right)\right\rangle\left|\psi_{-}\left(\mathbf{r}_{1}\right)\right\rangle\right]
$$

in Hartree-Fock approximation. 


\subsubsection{DM Interaction}

The electric dipole moment is given by

$$
\begin{aligned}
\mathbf{P} & =e\left\langle\psi\left|\mathbf{r}_{1}+\mathbf{r}_{2}\right| \psi\right\rangle=e\left(\frac{1}{\left\langle\psi_{+} \mid \psi_{+}\right\rangle}-\frac{1}{\left\langle\psi_{-} \mid \psi_{-}\right\rangle}\right)\left\langle\psi_{+}\left|\mathbf{r}_{1}\right| \psi_{+}\right\rangle \\
& =-\frac{4 e}{9}\left(\frac{t}{V}\right)^{3} I \hat{\mathbf{e}}_{12} \times\left(\mathbf{n}_{1} \times \mathbf{n}_{2}\right),
\end{aligned}
$$

where

$$
I=\int d^{3} r_{i} d_{y z}\left(\mathbf{r}_{i}+\frac{\mathbf{R}}{2}\right) y p_{z}(0), \quad(i=1,2)
$$

and its cyclic permutations. The integral $I$ is estimated to be $I \simeq \frac{16}{27} Z_{O}^{5 / 2} Z_{M}^{7 / 2}\left(\frac{Z_{O}}{2}+\right.$ $\left.\frac{Z_{M}}{3}\right)^{-6} a_{0}$, where $a_{0}$ is the Bohr radius and $Z_{O} / Z_{M}$ is the atomic number of $\mathrm{O} / \mathrm{M}$. The electric dipole moment interacts with the external electric field $\mathbf{E}$ and change the full Hamiltonian by a term of the DM form

$$
H_{D M}=-\mathbf{P} \cdot \mathbf{E}=\frac{4 e}{9}\left(\frac{t}{V}\right)^{3} I \hat{\mathbf{e}}_{12} \times\left(\mathbf{n}_{1} \times \mathbf{n}_{2}\right) \cdot \mathbf{E}=\frac{4 e}{9}\left(\frac{t^{4}}{V}\right) \frac{I}{t}\left(\mathbf{E} \times \hat{\mathbf{e}}_{12}\right) \cdot\left(\mathbf{n}_{1} \times \mathbf{n}_{2}\right) .
$$

Comparing it with the DM term (2.7) we obtained in Sec. 2.1

$$
H_{D M}=J_{e x} \frac{e a}{E_{S O}}\left(\mathbf{E} \times \hat{\mathbf{e}}_{12}\right) \cdot\left(\mathbf{S}_{1} \times \mathbf{S}_{2}\right)
$$

with $J_{e x}=\frac{t^{4}}{V^{3}}$, we arrive at an unambiguous identification of $E_{S O}$ within our model:

$$
E_{S O}=\frac{9 t a}{4 I}
$$


Here $a$ is the distance between neighboring magnetic ions. Taking YIG as an example $[18,19]$, with $t=0.8 \mathrm{eV}$, and $I=0.61 a$, we get $E_{S O}=3.0 \mathrm{eV}$. 


\section{Chapter 3}

\section{Electric Control of Phase Shift in Thin Films}

In this chapter, we apply an electric field in an insulating ferromagnetic thin film. Due to the Dzyaloshinskii-Moriya (DM) interaction developed in Chapter 2, the field induces a relative shift of wave vector $\delta k / k$, which can be as large as $1 \%$ with proper choosing of wave vectors - to achieve the maximum effect the wave vector, the equilibrium magnetization and the applied electric field must be perpendicular to each other.

We begin by developing the equation of motion (Landau-Lifshitz equation with DM interaction) for the system and then use the spin-wave mode method [21] to obtain the dispersion of the lowest lying mode allowed in the film. From the dispersion, we calculate the electric-field-induced phase shift in Sec. 3.3. As discovered in Chapter 2, the DM term produces a change of frequency linear in $\mathrm{k}$, which can be tested by the change of group velocity in the presence of an electric field: the possible experimental 


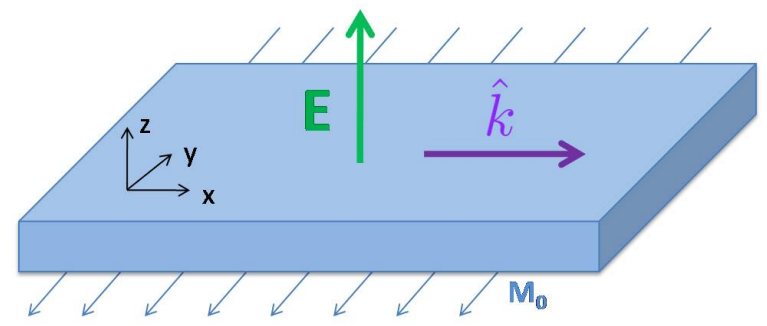

Figure 3.1: Schematic illustration of a tangentially magnetized film.

set up is also illustrated in Sec. 3.3.

\subsection{Model and equation of motion}

We consider a ferromagnetic film of a finite thickness $d$ in the $z$ direction as shown in Fig. 3.1. The Hamiltonian of the system is written in terms of the magnetization $\mathbf{M}(r, t)=\mathbf{M}_{0}+\mathbf{m}(\mathbf{r}, t)$, where $\mathbf{M}_{0}$ is the equilibrium magnetization and $\mathbf{m}(\mathbf{r}, t)$ is its deviation from equilibrium, as follows

$$
\mathscr{H}=\mathscr{H}_{\text {ex }}+\mathscr{H}_{a}+\mathscr{H}_{\text {dip }}+\mathscr{H}_{D M}+\mathscr{H}_{Z}
$$

where

$$
\mathscr{H}_{e x}=\frac{J}{2} \int d^{3} r|\nabla \mathbf{M}(\mathbf{r})|^{2}
$$

is the ferromagnetic exchange interaction,

$$
\mathscr{H}_{a}=\frac{\mu_{0}}{2} \int d^{3} r M_{z}^{2},
$$


is the shape anisotropy energy,

$$
\mathscr{H}_{\text {dip }}=\frac{\mu_{0}}{8 \pi} \int d^{3} r d^{3} r^{\prime} \frac{[\boldsymbol{\nabla} \cdot \mathbf{m}(\mathbf{r}, t)]\left[\boldsymbol{\nabla}^{\prime} \cdot \mathbf{m}\left(\mathbf{r}^{\prime}, t\right)\right]}{\left|\mathbf{r}-\mathbf{r}^{\prime}\right|}
$$

is the dipole-dipole interaction in the magnetostatic approximation,

$$
\mathscr{H}_{D M}=\int d^{3} r \mathbf{D} \cdot[\mathbf{M}(\mathbf{r}) \times \nabla \mathbf{M}(\mathbf{r})]
$$

is the electric-field induced Dzyaloshinskii-Moriya (DM) interaction, discussed in Chapter 2 and later in this section, and

$$
\mathscr{H}_{Z}=-\mu_{0} \int d^{3} r \mathbf{H}_{0} \cdot \mathbf{M}
$$

is the Zeeman energy. In the above formulas $\mu_{0}=4 \pi \times 10^{-7} \mathrm{~T} \cdot \mathrm{m} / \mathrm{A}$ is the magnetic permeability of the vacuum. Notice that the exchange coupling $J$ has the dimension of $\mu_{0} \cdot \mathrm{m}^{2}$, and, accordingly, the DM vector, $D$, has the dimension of $\mu_{0} \cdot \mathrm{m}$.

The dynamics of the magnetization is described by the Landau-Lifshitz equation

$$
\frac{\partial \mathbf{M}(\mathbf{r}, t)}{\partial t}=\gamma \mathbf{B}_{e f f} \times \mathbf{M}(\mathbf{r}, t) .
$$

The effective magnetic field

$$
\mathbf{B}_{e f f}=-\frac{\partial \mathscr{H}}{\partial \mathbf{M}}
$$

is derived from the Hamiltonian of the system. We give below the effective magnetic fields corresponding to the interactions listed above. 
The exchange field is given by

$$
\mathbf{B}_{e x}=J \nabla^{2} \mathbf{M}(\mathbf{r}) .
$$

The shape anisotropy and the magnetic dipole-dipole interaction together yield the demagnetizing field,

$$
\mathbf{B}_{d}=-\mu_{0} M_{0 z} \hat{\mathbf{e}}_{z}+\mu_{0} \mathbf{h}_{d i p}(\mathbf{r}, t),
$$

where $M_{0 z}$ is the $z$-component of the equilibrium magnetization, $\hat{\mathbf{e}}_{z}$ is perpendicular to the film as shown in Fig. 3.1, and

$$
\mathbf{h}_{d i p}(\mathbf{r}, t)=\frac{1}{4 \pi} \boldsymbol{\nabla} \int d^{3} r^{\prime} \frac{\boldsymbol{\nabla}^{\prime} \cdot \mathbf{m}\left(\mathbf{r}^{\prime}, t\right)}{\left|\mathbf{r}-\mathbf{r}^{\prime}\right|}
$$

Notice that the equilibrium magnetization induces a constant demagnetizing field, while the spin waves induce a time-dependent demagnetizing field, which we shall discuss in detail later.

As mentioned in Chapter 2, the DM term stems from the SO coupling of the electrons that mediate the interaction between the magnetic ions to the external electric field $\mathbf{E}$. We get

$$
\mathbf{B}_{D M}=2 \mathbf{D} \times\left(\hat{\mathbf{e}}_{i j} \cdot \nabla\right) \mathbf{M},
$$

where

$$
\mathbf{D}=J \frac{e \mathbf{E} \times \hat{\mathbf{e}}_{i j}}{E_{s o}}
$$

is the DM vector, perpendicular to both the electric field and the unit vector $\hat{\mathbf{e}}_{i j}$ along 
the line that connects the magnetic ions. Here $J$ is the Heisenberg exchange coupling, defined precisely in Eq. (3.2), $e$ is the absolute value of the electron charge, and $E_{s o}$ is an energy scale associated with the inverse of the strength of the spin-orbit coupling, i.e. $E_{s o}$ is large when spin-orbit interaction is weak and vice versa. For electrons in vacuum $E_{s o}$ would be of the order of the electron rest energy $m c^{2} \sim 5 \times 10^{5} \mathrm{eV}$, rendering $\mathbf{D}$ negligibly small. In the case of Fe ions, however, the strong spin-orbit entanglement that is built into the relevant $d$ orbitals creates a much smaller value of $E_{s o}$, namely $E_{s o} \simeq t$ where $t$ is the hopping amplitude between Fe and $\mathrm{O}$ ions, which is typically of the order of $1 \mathrm{eV}$.

Substituting the effective magnetic fields (3.9), (3.10), (3.12) and the external magnetic field (Zeeman term) into Eq. (3.7), we obtain the equation of motion of the magnetization

$$
\begin{aligned}
& \frac{\partial \mathbf{M}}{\partial t}=-\gamma \mathbf{M} \times\left[J \nabla^{2} \mathbf{M}-\mu_{0}\left(M_{0 z} \hat{\mathbf{e}}_{z}+\mathbf{h}_{d i p}+\mathbf{H}_{0}\right)\right] \\
& +2 \gamma(\mathbf{D} \cdot \mathbf{M})\left(\hat{\mathbf{e}}_{i j} \cdot \nabla\right) \mathbf{M} .
\end{aligned}
$$

Expanding to first order in $\mathbf{m} \equiv \mathbf{M}-\mathbf{M}_{0}$, and making use of the fact that the magnitude of $\mathbf{M}$ is constant (which implies $\mathbf{m}$ perpendicular to $\mathbf{M}_{0}$ ) we get

$$
\begin{aligned}
& \frac{\partial \mathbf{m}}{\partial t}-\omega_{M} \lambda_{E}\left(\hat{\mathbf{e}}_{i j} \cdot \nabla\right) \mathbf{m}=-\omega_{M} \mathbf{n}_{0} \times\left(\lambda_{e x}^{2} \nabla^{2} \mathbf{m}+\mathbf{h}_{d i p}\right) \\
& -\mathbf{m} \times\left(\omega_{H} \mathbf{n}_{0}-\omega_{M} n_{z} \hat{\mathbf{e}}_{z}\right),
\end{aligned}
$$

where $\mathbf{n}_{0}$ denotes the unit vector in the direction of $\mathbf{M}_{0}, n_{z}=\frac{M_{0 z}}{M_{0}}$, and the external magnetic field is assumed to be directed along $\mathbf{n}_{0}$. We have introduced the following 
notation:

$$
\begin{aligned}
& \omega_{M}=\gamma \mu_{0} M, \quad \omega_{H}=\gamma \mu_{0} H, \\
& \lambda_{E}=\frac{2 J e\left(\mathbf{E} \times \hat{\mathbf{e}}_{i j}\right) \cdot \mathbf{n}_{0}}{\mu_{0} E_{S O}}, \quad \lambda_{e x}=\sqrt{\frac{J}{\mu_{0}}},
\end{aligned}
$$

Eq. (3.15) shows that the DM interaction takes effect only if the $\mathbf{E}$ field, the wave vector of the spin waves and the equilibrium magnetization are perpendicular to each other.

Due to shape anisotropy, a tangential magnetization appears naturally in magnetic films. Tangentially magnetized films are particularly convenient for the excitation and propagation of exchange spin waves. [4] We therefore focus on this kind of film and discuss the $\mathbf{E}$-induced phase shift of the spin waves propagating in two special directions: perpendicular (transverse spin waves) and parallel (longitudinal spin waves) to the equilibrium magnetization. The above discussion implies that the longitudinal spin waves (BVMSWs at long wavelength) are unaffected by the electric field since their wave vector is parallel to $\mathbf{n}_{0}$, while the transverse spin waves are affected more significantly than those propagating in any other directions. In Secs. 3.2 and 3.3, we shall obtain the dispersion of the transverse spin waves and quantify the effect of the E field on their propagation.

\subsection{Dispersion of Transverse Spin Waves in a mag- netic film}

Let us assume that the equilibrium magnetization is oriented along the negative $y$ axis, $\mathbf{n}_{0} \|-\hat{\mathbf{e}}_{y}$, while the spin wave propagates along $x$-axis (see Fig. 3.1 ). The 
oscillating part of the magnetization has the form

$$
\mathbf{m}(x, z, t)=\sum_{k}\left\{\left[m_{k, x}(z) \hat{\mathbf{e}}_{x}+m_{k, z}(z) \hat{\mathbf{e}}_{z}\right] \exp [i(\omega t-k x)]\right\}
$$

where $k$ and $\omega$ are the wave vector and the frequency of the wave, and $m_{k, x}(z), m_{k, z}(z)$ are the amplitudes of the oscillation along the $x$ and $z$ axes respectively.

The $z$-dependence of the magnetization arises from the dipole field derived earlier, see Eq. (3.11)

$$
\mathbf{h}_{d i p}(\mathbf{r})=\frac{1}{4 \pi} \nabla \int \frac{\nabla^{\prime} \cdot \mathbf{m}(\mathbf{r}) d \mathbf{r}^{\prime}}{\left|\mathbf{r}-\mathbf{r}^{\prime}\right|}
$$

which, for a periodic field of wave vector $k$ in the $x$ direction takes the form

$$
h_{k, i}=\sum_{j=x, z} \int_{-d / 2}^{d / 2} G_{i j}\left(z, z^{\prime}\right) m_{k, j}\left(z^{\prime}\right) d z^{\prime}
$$

where the film lies between the planes $z=-d / 2$ and $z=d / 2, i$ and $j$ are cartesian indices ranging over components $x$ and $z$, and $G_{i j}$ is the two-dimensional matrix

$$
\mathbf{G}\left(z, z^{\prime}\right)=\left(\begin{array}{cc}
-G_{p}\left(z, z^{\prime}\right) & i G_{Q}\left(z, z^{\prime}\right) \\
i G_{Q}\left(z, z^{\prime}\right) & G_{p}\left(z, z^{\prime}\right)-\delta\left(z-z^{\prime}\right)
\end{array}\right)
$$

with $G_{p}\left(z, z^{\prime}\right)=\frac{|k|}{2} \exp \left[-|k|\left|z-z^{\prime}\right|\right]$ and $G_{Q}\left(z, z^{\prime}\right)=\frac{k}{2} \exp \left[-|k|\left|z-z^{\prime}\right|\right] \operatorname{sgn}\left(z-z^{\prime}\right)$. [21] 
Now Eq. (3.15) is rewritten in matrix form

$$
\begin{aligned}
& \left(\begin{array}{cc}
\frac{\omega_{H}}{\omega_{M}}+\lambda_{e x}^{2}\left(k^{2}-\partial_{z}^{2}\right) & -i\left(\frac{\omega}{\omega_{M}}+\lambda_{E} k\right) \\
i\left(\frac{\omega}{\omega_{M}}+\lambda_{E} k\right) & \frac{\omega_{H}}{\omega_{M}}+\lambda_{e x}^{2}\left(k^{2}-\partial_{z}^{2}\right)
\end{array}\right)\left(\begin{array}{l}
m_{k, x}(z) \\
m_{k, z}(z)
\end{array}\right) \\
& =\int_{-d / 2}^{d / 2} \mathbf{G}\left(z, z^{\prime}\right)\left(\begin{array}{c}
m_{k, x}\left(z^{\prime}\right) \\
m_{k, z}\left(z^{\prime}\right)
\end{array}\right) d z^{\prime} .
\end{aligned}
$$

Since the DM term $\lambda_{E} k$ appears everywhere as an additive correction to $\frac{\omega}{\omega_{M}}$, it follows that the effect of the electric field can be taken into account simply by replacing $\omega$ with $\omega+\omega_{M} \lambda_{E} k$ in the $\mathbf{E}=0$ dispersion.

Let us now focus on the $\mathbf{E}=0$ dispersion. We apply the spin wave modes method of Ref. [21]. The $z$-dependence of the magnetization is expanded in cosine waves as follows:

$$
\mathbf{m}_{k}(z)=\sum_{n=0}^{\infty} \mathbf{m}_{n} \cos \left[q_{n}\left(z+\frac{d}{2}\right)\right]
$$

where $q_{n}=\frac{n \pi}{d}(n=0,1,2, \ldots)$ in order to enforce "unpinned" exchange boundary condition, whereby the derivative of the magnetization in the $z$ direction vanishes at the surfaces of the film: $\left.\frac{d \mathbf{m}(z)}{d z}\right|_{z= \pm d / 2}=0$.

Note that cosine waves with different values of $n$ are connected by the $\mathbf{G}$ matrix, which comes from the small demagnetizing field created by the spin waves. However, the mixing of cosine waves with different values of $n$ tends to zero in the limits of $k d \ll 1$ and $k d \gg 1$. Thus, in a first approximation we can neglect the mixing of different cosine waves. For example, in this approximation the main mode, $n=0$, in 


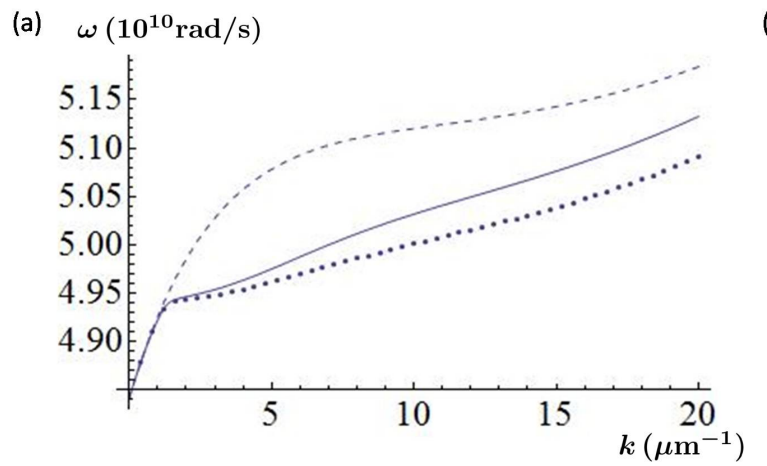

(b) $\omega\left(10^{10} \mathrm{rad} / \mathrm{s}\right)$

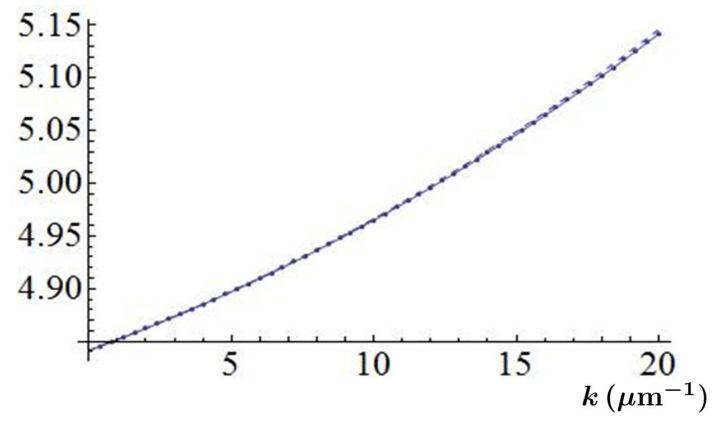

Figure 3.2: Comparison of the dispersions obtained from zeroth order perturbation theory (dashed line), first order degenerate perturbation theory (solid line), and numerical diagonalization (dotted line) in the absence of electric field: (a) $d=0.2 \mu \mathrm{m}$; (b) $d=0.02 \mu \mathrm{m}$.

which the magnetization is uniform across the thickness of the film, has the dispersion

$$
\begin{array}{cl}
\omega_{T 0}=\quad & \left\{( \omega _ { H } + \omega _ { M } \lambda _ { e x } ^ { 2 } k ^ { 2 } + \omega _ { M } \frac { 1 - e ^ { - | k | d } } { | k | d } ) \left[\omega_{H}+\omega_{M} \lambda_{e x}^{2} k^{2}\right.\right. \\
& \left.\left.+\omega_{M}\left(1-\frac{1-e^{-|k| d}}{|k| d}\right)\right]\right\}^{1 / 2}-\omega_{M} \lambda_{E} k,
\end{array}
$$

where $\omega_{H}=3.53 \times 10^{10} \mathrm{rad} / \mathrm{s}, \omega_{M}=3.11 \times 10^{10} \mathrm{rad} / \mathrm{s}, \lambda_{e x}=113 \AA$ and $\lambda_{E}=\frac{-2 \mathrm{JeE}}{\mu_{0} E_{S O}}$. Here, we have used the parameters previously estimated for YIG and considered it as a simple cubic ferromagnet with lattice constant $a$, which is valid when the excitation energy is below $40 \mathrm{~K}$. [18] We have also assumed that the $\hat{\mathbf{e}}_{i j}$ direction in Eq. (3.16) coincides with the direction of propagation of the wave, $\hat{\mathbf{x}}$. Notice that, as anticipated in Chapter 2, the electric field enters only through the last term of Eq. (3.22) - a linear-in- $k$ shift of the frequency. This shift is actually exact regardless of the approximations we made in arriving at Eq. (3.22). It is also independent of the index $n$ of the cosine wave. 
The main drawback of the zeroth order perturbation theory described in the previous paragraph is that it predicts nonphysical crossings between branches of spin waves characterized by different values of $n$. To correct this problem we resort to exact diagonalization, i.e., we numerically solve the eigenvalue problem (3.20) on a basis of 20 cosine waves, including the $n=0$ mode. In Fig. 3.2, we compare the "exact" dispersion with the dispersion obtained from the zeroth-order perturbation and that from first-order degenerate perturbation in which Eq. (3.20) is solved on a basis that only includes the $n=0$ mode and the cosine waves that have crossings with it at finite $k$. We see that the first-order theory works pretty well in the limit of $k d \ll 1$, that is to say, either in the long wavelength limit (Fig. 3.2(a)) or when the film is very thin (Fig. 3.2(b)). In particular, the slope of the dispersion of the $n=0$ mode at $k=0$ remains unchanged and equals to the zeroth order result. Furthermore, the dispersion from the first-order theory qualitatively agrees with the exact solution. Therefore, it is safe to use the first-order theory. The results plotted in Figs. 3.3 and 3.4 have been obtained by this approach.

Figs. 3.3 and 3.4 show the evolution of the dispersion from the magnetostaticdominated regime $(k d \ll 1)$ to the exchange-dominated regime $(k d \gg 1)$. When $k \rightarrow 0$, the dispersion shows linear behavior even without applying the electric field, due to the dipolar interaction (see the dip in Fig. 3.4). The slope of the dispersion, also known as the group velocity of the spin waves, tends to $\omega_{M}^{2} d /\left(4 \sqrt{\omega_{H}\left(\omega_{H}+\omega_{M}\right)}\right)$ for $k \rightarrow 0$, and will be changed by $-\omega_{M} \lambda_{E}$ in the presence of the $\mathbf{E}$ field. This effect is large enough to be observed when $\left|\lambda_{E}\right| \sim 0.01 d$, that is to say when $|\mathbf{E}| \sim 10^{7} \mathrm{~V} / \mathrm{m}$ at $d=1 \mu \mathrm{m}$. The linear-in- $k$ dispersion at small $k$ is more efficiently tuned in a thin film than in a thick one (see Fig. $3.4(\mathrm{~b})$ ). This can be understood by noting that 


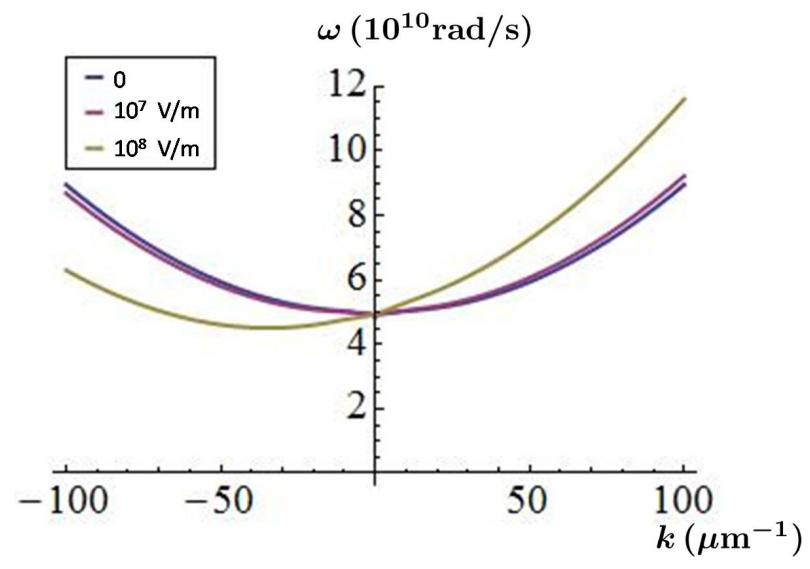

Figure 3.3: Dispersion of spin waves propagating in a tangentially magnetized film of thickness $d=0.2 \mu \mathrm{m}$. The curves are presented in different strengths of electric field as shown in the legend.

(a)

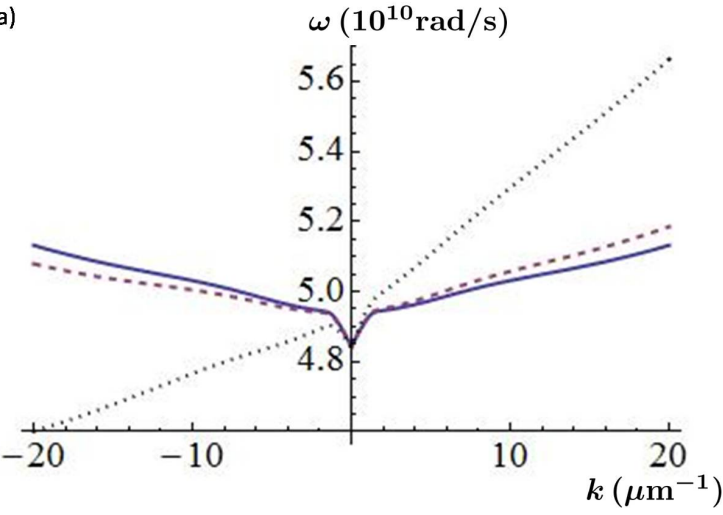

(b)

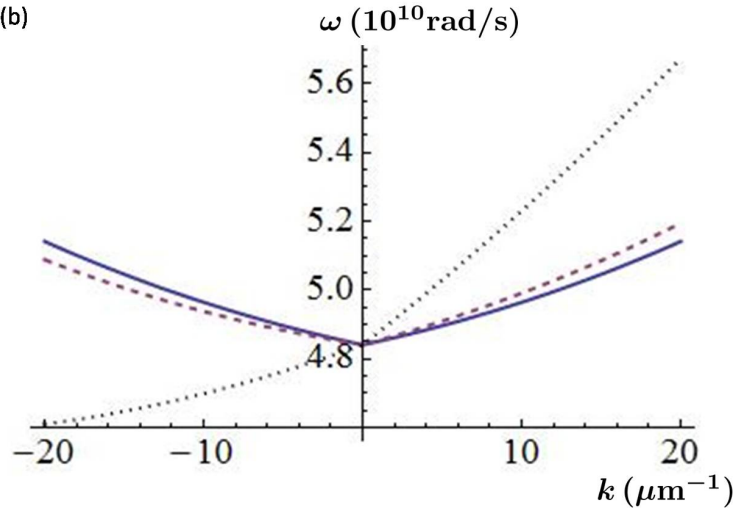

Figure 3.4: Dispersion of spin waves propagating in a tangentially magnetized film of thickness (a) $d=0.2 \mu \mathrm{m}$, (b) $d=0.02 \mu \mathrm{m}$. The curves are presented for different strengths of electric field: $E=0$ (solid), $10^{7} \mathrm{~V} / \mathrm{m}$ (dashed) and $10^{8} \mathrm{~V} / \mathrm{m}$ (dotted). 


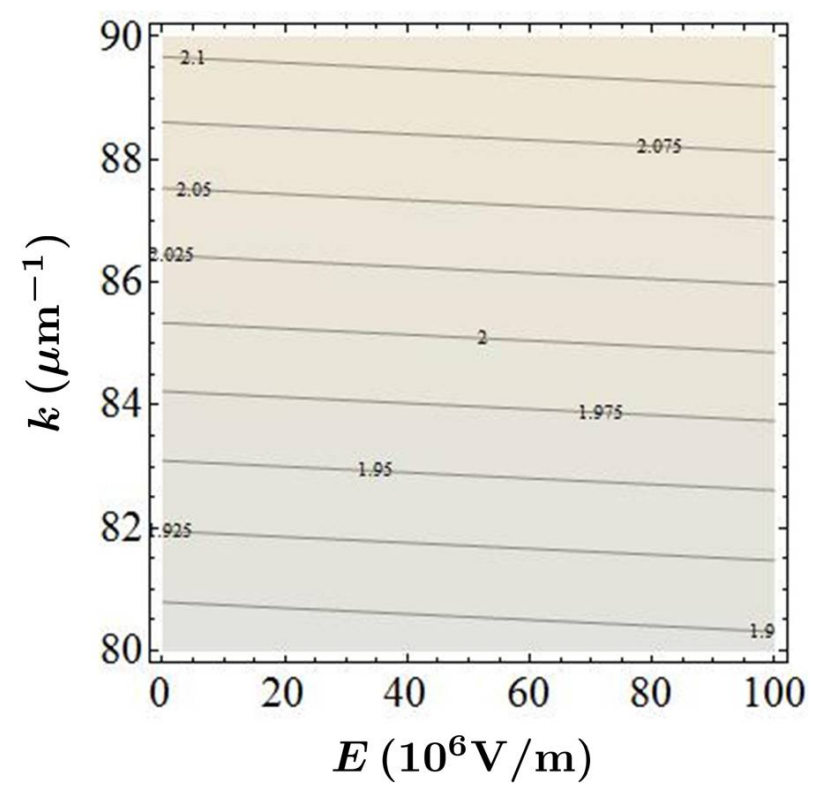

Figure 3.5: Wave vector as a function of electric field in a $0.02-\mu \mathrm{m}$ thick tangentially magnetized film. The frequency of the wave is shown on each curve and is expressed in units of $\omega_{M}=3.11 \times 10^{10} \mathrm{rad} / \mathrm{s}$.

the dipolar interaction is minute compared with the exchange interaction, but it is long-ranged while the latter is short-ranged. Therefore, the dipolar interaction plays a more and more important role as the thickness of the films increases, but can be neglected in very thin films, i.e., for thicknesses smaller than about $0.01 \mu \mathrm{m}$. For such films, the small- $k$ behavior of the dispersion is controlled by the electric field.

\subsection{Electric-field induced phase shift}

The above analysis suggests that an electric field-controlled phase shifter for spin waves could be realized in a thin film with tangential magnetization. Now let us focus on the film as shown in Fig. 3.1 and set the film thickness to be $0.02 \mu \mathrm{m}$. 


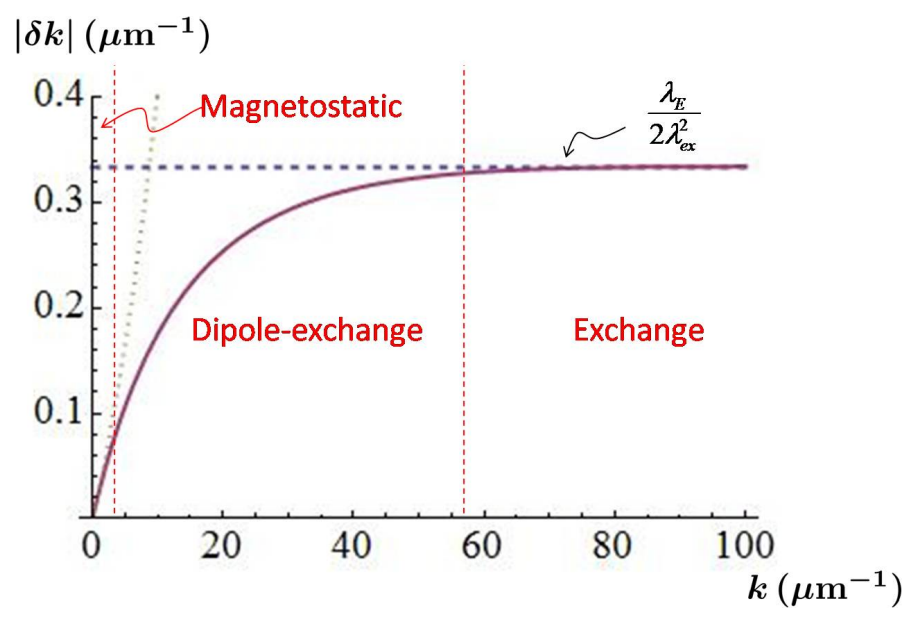

Figure 3.6: Solid line: variation of wave vector $|\delta k|=|k(E)-k(0)|$ in a $0.02-\mu \mathrm{m}$ thick tangentially magnetized film in the presence of an electric field $E=10^{6} \mathrm{~V} / \mathrm{m}$. The black dotted line is calculated from considering exchange interaction only.

Noting that $\omega$ is a function of $k$ and $E$, we calculate the shift of wave vector $(\delta k)$ due to the applied electric field at a given frequency by requiring $d \omega=0$. Fig. 3.5 shows the linear relation between the wave vector of the spin wave and the applied $\mathbf{E}$ field at given frequencies. Fig. 3.6 shows a plot of electric field-induced $\delta k$ vs $k$ for $E=10^{6}$ $\mathrm{V} / \mathrm{m} . \delta k$ grows rapidly with increasing $k$ in the magnetostatic regime $(\delta k / k \sim 1 \%)$, and tends to a limiting value $\lambda_{E} /\left(2 \lambda_{e x}^{2}\right)$ (independent of $\left.d\right)$ in the exchange regime. (This is also the value one finds at any $k$ if one neglects the dipole field altogether.) With these results in hand, we see that a $\pi$-phase-shift can be obtained for a spin wave of wave vector $9 \mu \mathrm{m}^{-1}$ propagating over a distance of $L=20 \mu \mathrm{m}$ in the presence of an electric field $E=10^{6} \mathrm{~V} / \mathrm{m}$.

In addition to the possible application to controlled phase shifter, we believe that the observation of an electric field-induced change in the group velocity of spin waves would be of fundamental interest. The measurement could be done in the apparatus 


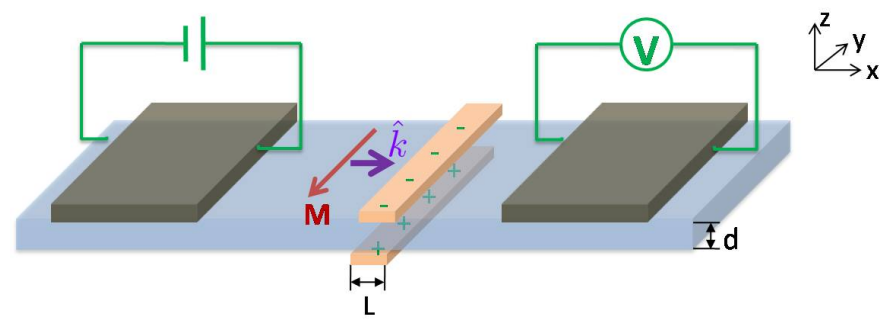

Figure 3.7: Sketch of possible experimental set up for testing the effect of the electric field on the group velocity of spin waves. A tangentially magnetized YIG film (blue) is sandwiched between the plates of a capacitor that provides the electric field, which induces a change of the group velocity. The spin waves can be excited by spin transfer torque[3] and then be measured by inverse spin Hall effect[3].

schematically illustrated in Fig. 3.7, where spin waves are injected by spin transfer torque at one end of a YIG waveguide and the change of group velocity is obtained from the measured delay in the arrival time of the spin wave signal at the other end of the waveguide in the presence of the electric field. 


\section{Chapter 4}

\section{Spin-Wave Interferometer: Voltage-Controlled NOT Gate}

A crucial element of magnonics [1] is the phase shifter - a device that changes the phase of propagating spin waves. Several mechanisms have been proposed in the past to implement controlled phase shifts on spin waves. The simplest and most direct, is the application of a magnetic field, which shifts the dispersion [22], thus changing the wave vector at constant frequency [5]. More sophisticated mechanisms exploited the Berry phase accumulated by spin waves that propagate on a non-collinear magnetic texture $[23,24]$. In this chapter, we study the spin waves propagating in a onedimensional ring in the presence of a radial electric field. It is worth noting that, after traveling along the ring, the spin wave acquires not only an electric-field-induced Aharanov-Casher (AC) but also a geometric phase. It is because the equilibrium magnetization of the ring is not homogeneous but a texture due to shape anisotropy. 


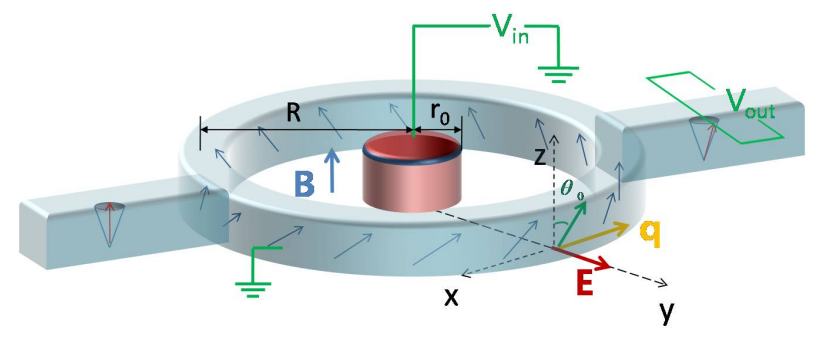

Figure 4.1: A Mach-Zehnder spin-wave interferometer in the presence of radial $\mathbf{E}$ field. A weak magnetic field is applied perpendicular to the ring plane, tilting the equilibrium magnetization away from the ring but still in the tangential plane to the ring. $\theta_{0}$ denotes the orientation of the equilibrium magnetization.

\subsubsection{Spin wave dispersion}

As the size of magnets shrinks to nanometer scale or sub-micrometer scale, it is the exchange interaction determines the dispersion of spin waves. Hence, we will drop the magnetic dipolar interaction in Eq. (3.1) and require the shape anisotropy to be along the ring.

We now proceed to solve the dispersion of the spin waves in the presence of the DM interaction derived in Chapter 2. We consider the ring geometry illustrated in Fig.4.1: the electric field perpendicular to the ring produces a DM vector $\mathbf{D}$ directed along the $z$-axis. This will affect the dispersion of spin waves if and only if the equilibrium magnetization has a non-vanishing component along the $z$ axis.

In a flat ring, such as the one shown in Fig.4.1, the shape anisotropy $-K(\mathbf{M}$. $\hat{\mathbf{e}})^{2} / M^{2}$ where $\hat{\mathbf{e}}$ is the unit vector along the ring - outweighs other forms of anisotropy, causing the equilibrium magnetization to lie along the ring, in which case the electric field has no influence. As a result, a magnetic field along the $z$ axis (Zeeman coupling $\left.B M_{z}\right)$ is necessary for us to observe the impact of the DM term on spin waves propagating in the ring. Now, however, the orientation of the equilibrium magnetization is 
no longer constant in absolute space (even though it is constant relative to the ring). This causes an additional geometric phase $\left(\alpha_{g}=\frac{a}{R}\right)$ to appear, as shown in Ref. [23], where $R$ is the radius of the ring. Putting everything together, i.e., DM interaction, geometric phase, Zeeman coupling and shape anisotropy, we arrive at the following equation of motion:

$$
\frac{\partial \mathbf{M}}{\partial t}=-\gamma \mathbf{M} \times\left[J \partial_{x}^{2} \mathbf{M}-\mu_{0} M^{x} \hat{\mathbf{e}}_{x}-B \hat{\mathbf{e}}_{z}\right]+2 \gamma D_{z} M^{z} \partial_{x} \mathbf{M}
$$

The large magnitude of the "block spin" of YIG $(\mathrm{S}=14.3)$ allows us to use the semiclassical spin-wave approach, where the spin fluctuation is in the plane perpendicular to $M_{0}$. Hence, it is convenient to introduce a new reference frame that z-axis is always along the direction of $M_{0}$. We are going to use tilde to denote everything in the new reference frame, where $\mathbf{M}=\mathbf{R}^{-1} \tilde{\mathbf{M}}$ with $\mathbf{R}^{-1}$ being the rotation matrix around $y$ axis as shown in Fig. 4.1

$$
\begin{aligned}
\mathbf{R}^{-1} & =\left(\begin{array}{ccc}
\cos \theta & 0 & -\sin \theta \\
0 & 1 & 0 \\
\sin \theta & 0 & \cos \theta
\end{array}\right) . \\
M^{x} & =\cos \theta \tilde{M}_{x}+\sin \theta M, \\
M^{z} & =\sin \theta \tilde{M}_{x}-\cos \theta M, \\
\hat{\mathbf{e}}_{x} & =\cos \theta \tilde{\hat{\mathbf{e}}}_{x}-\sin \theta \tilde{\hat{\mathbf{e}}}_{z}, \\
\hat{\mathbf{e}}_{z} & =\sin \theta \tilde{\hat{\mathbf{e}}}_{x}+\cos \theta \tilde{\hat{\mathbf{e}}}_{z} .
\end{aligned}
$$

where $M$ is the magnitude of magnetization. By using Eq. (4.2)-(4.6), we get the 
Landau-Lifshitz equation in the new reference frame,

$$
\begin{aligned}
\frac{\partial \tilde{\mathbf{M}}}{\partial t}= & -\gamma \tilde{\mathbf{M}} \times\left[J \partial_{x}^{2} \tilde{\mathbf{M}}-\mu_{0}\left(\cos \theta \tilde{M}_{x}-\sin \theta M\right)\left(\cos \theta \tilde{\hat{\mathbf{e}}}_{x}-\sin \theta \tilde{\hat{\mathbf{e}}}_{z}\right)\right. \\
& \left.-B\left(\sin \theta \tilde{\hat{\mathbf{e}}}_{x}+\cos \theta \tilde{\hat{\mathbf{e}}}_{z}\right)\right]+2 \gamma D_{z}\left(\sin \theta \tilde{M}_{x}+\cos \theta M\right) \partial \tilde{\mathbf{M}}
\end{aligned}
$$

Notice that the transform of $\partial_{x}^{2} \mathbf{M}$ is tricky, you should go back to the Hamiltonian and remember the effective magnetic field in the new reference frame is $-\partial \mathscr{H} / \partial \tilde{\mathbf{M}}$ instead of substituting $\mathbf{M}=\mathbf{R}^{-1} \tilde{\mathbf{M}}$ into Eq. (4.1). In the assumption of plane wave solution $e^{i(k x-\omega t)}$, we have

$$
\begin{aligned}
-i \omega \tilde{M}_{x}= & -\gamma \tilde{M}_{y}\left[-\mu_{0} M \sin ^{2} \theta-B \cos \theta\right]+\gamma J M \partial_{x}^{2} \tilde{M}_{y}+2 \gamma D_{z} M \cos \theta \partial_{x} \tilde{M}_{x} \\
-i \omega \tilde{M}_{y}= & -\gamma M\left[J \partial_{x}^{2} \tilde{M}_{x}-\mu_{0} \cos ^{2} \theta \tilde{M}_{x}+\mu_{0} M \sin \theta \cos \theta-B \sin \theta\right] \\
& +\gamma \tilde{M}_{x}\left[-\mu_{0} M \sin ^{2} \theta-B \cos \theta\right]+2 \gamma D_{z} M \cos \theta \partial_{x} \tilde{M}_{y} .
\end{aligned}
$$

By using the equilibrium condition $\cos \theta_{0}=\frac{B}{\mu_{0} M}$ and solving the secular equation

$$
\operatorname{det}\left|\begin{array}{cc}
2 \gamma D_{z} M \cos \theta_{0} i k+i \omega & -\gamma \mu_{0} M-\gamma J M k^{2} \\
\gamma \mu_{0} M \sin ^{2} \theta+\gamma J M k^{2} & 2 \gamma D_{z} M \cos \theta i k+i \omega
\end{array}\right|=0
$$

we arrive at the spin-wave dispersion:

$$
\omega=J \gamma M\left\{\sqrt{\left(k^{2}+\kappa^{2}\right)\left(k^{2}+\kappa^{2} \sin ^{2} \theta_{0}\right)}+2 \bar{\alpha} k \cos \theta_{0}\right\}
$$

where $\bar{\alpha}=\frac{e E a}{E_{S O}}-\alpha_{g}, \kappa=\sqrt{\frac{\mu_{0}}{J}}$, and $\cos \theta_{0}=\frac{B}{\mu_{0} M}$, which is determined by minimizing the total Hamiltonian in the limit of $\kappa^{2} \gg \frac{1}{R^{2}}$.

As shown in Fig. 4.2, one can tune the dispersion by adjusting the electric and the 
(a)

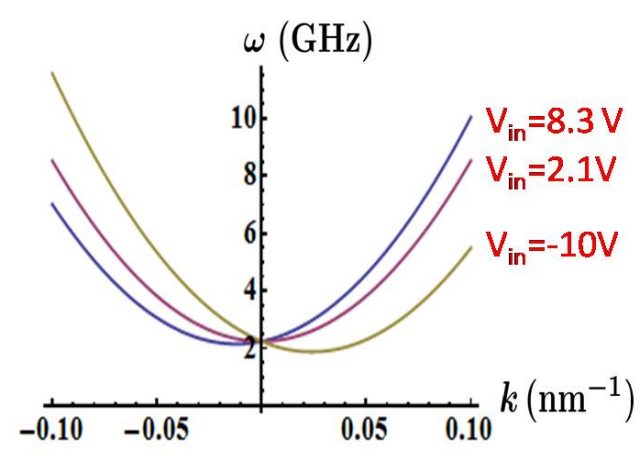

(b)

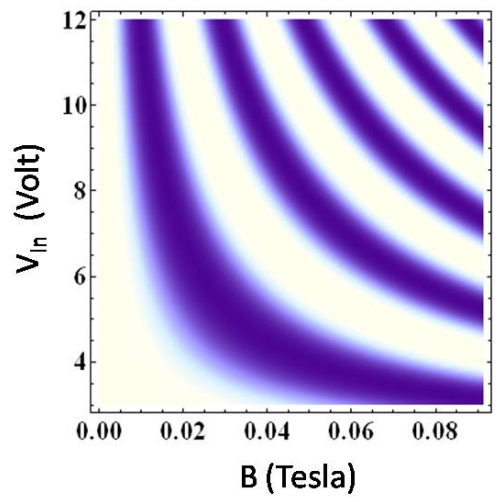

Figure 4.2: (a) Dispersion of spin waves in the ferromagnetic ring in Fig.4.1, taking into account the geometric phase and the phase induced by the electric field. Parameters we use $[18,19]: J^{\prime}=1.18 \times 10^{-4} \mathrm{eV}, K=1.53 \times 10^{-4} \mathrm{eV}, S=14.3$, $a=12.4 \AA, r_{0}=50 \mathrm{~nm}, R=100 \mathrm{~nm}, B=0.05 \mathrm{~T}$. (b) Transmission probability of a spin wave in the ring interferometer as a function of input voltage and magnetic field at $\omega=43 \mathrm{GHz}$.

magnetic fields. Just as a magnetic field shifts the spin wave dispersion vertically by increasing or decreasing the frequency at fixed $k$, the electric field shifts the dispersion horizontally by increasing or decreasing the wave vector at fixed frequency.

\subsubsection{Spin wave interferometer}

Now we are ready to design our spin-wave interferometric device. An insulating ring encircles a metal electrode to which a voltage $V_{i n}$ can be applied. The radial electric field acting upon the electrons in the ring is $\frac{-V_{i n}}{R \ln \left(r_{0} / R\right)}$.

In Fig. 4.2 (b) we plot the transmission of a spin wave sent through this MachZehnder interferometer, as a function of $V_{i n}$ and B. The effect of $B$ is to change the equilibrium orientation of the magnetization. The white regions in the figure are regions of constructive interference, separated by regions of destructive interference. 
We see that very modest changes of potentials and magnetic fields, of the order of $1 \mathrm{~V}$ and $0.01 \mathrm{~T}$ respectively, switch the response of the interferometer from high to low. It is then clear how the device can be used as a logic inverter: the logic input being the voltage on the central electrode, and the logic output the intensity of the spin wave, as measured by an inductive coupler. Advantages of this design are that it would operate at room temperature and $\mathrm{GHz}$ frequencies, with very little dissipation, and can be made small by using exchange spin waves - the only type we are really considering here, since magnetostatic spin waves have much longer wavelengths and are hardly affected by the AC phase. Once a logic inverter is available, we can follow Kostylev et al. [5] in constructing more complicated architectures, which implement the NAND, the NOR, and all of classical logic. 


\section{Chapter 5}

\section{Spin-Wave Drag}

In a conducting bilayer system, a steady current in the active layer produces a charge accumulation in the passive layer through Coulomb interaction. The resulting transverse resistivity gives information on electron-electron interaction, regardless of the interactions in the passive layer. This effect is named as Coulomb drag, since the redistribution of the charges in the passive layer is due to the drag of the moving charges in the active layer. In analogy to Coulomb drag, we propose here a spinwave drag in an insulating ferromagnetic bilayer system to separate the magnetic dipolar interaction from intralayer magnon-phonon interaction and magnon-magnon interactions due to exchange interactions. This idea is different from the spin-wave (magnon) drags presented in previous studies [25, 26, 27, 28, 29, 30].

The concept of spin-wave (magnon) drag was first used in the study of Seebeck effect (see Chap. 1) in ferromagnetic metals [25, 26] to explain the extra peaks, which cannot be explained by phonon-drag, appeared in the thermopower at low temperature. The idea is that the moving magnons under temperature gradient exchange 
momentum with electrons via magnon-electron interaction, which leads to an increase in the electron mobility and thus an increase in thermopower. However, since magnon and phonon share a lot of common features, such as they are both bosonic quasi-particles, it is very hard to separate the magnon contribution from the phonon one, especially in weak magnetic fields. Only recently, Costache et al realized a direct observation of the magnon drag effect in a thermopile formed by parallel ferromagnetic metal wires [29].

Another concept of magnon related drag appeared in a recent theoretical work [30]. The authors predicted an electric current drag effect in a NM/FI/NM layered structure (where NM stands for normal metal, and FI for ferromagnetic insulator). The idea is that due to the tunneling of spin current carried by magnons through the FI layer, the current running through one NM layer may induce an electric field in the other NM layer. The key mechanism here is the spin transfer torque exerted by the spin accumulation at NM/FI interface to the magnons in the FI layer.

In this chapter, we introduce a third type of magnon drag inspired by the Coulomb drag to study the magnetic dipolar interaction, since in most of the experiments and the applications in spin-wave spintronics the excited spin waves have relatively long wavelength and the dipolar interaction easily overwhelms the exchange interaction. Due to the interlayer dipolar interaction, we expect to observe a gradient of magnetization in the passive layer induced by a steady magnon current in the active layer. It is shown that the magnitude of the gradient achieves maximum when the driving current is parallel to the saturation magnetization, and minimum when they are perpendicular to each other. Temperature dependence of the drag resistivities reflects the characteristic of Bose-Einstein statistics. Since magnons are bosonic quasi-particles 
whose number may not be conserved. Our magnon drag shows both similarities and differences compared with the drag effect in cold atoms, in which the particle number is conserved. In particular, with decreasing temperature, our drag resistivity first increases as $1 /(\ln T)^{2}$ and then drops to zero because the magnon number tends to zero at low temperature, while the cold-atom drag resistivity increases as $1 / T^{2}$ monotonously until forming Bose-Einstein condensation.

The chapter is organized as follows: Sec. 5.1 briefly introduces how to get spinwave quanta (magnon) via Holstein-Primakoff transformation. The magnon current density and the heat current density are expressed in terms of out-of-equilibrium magnon distribution in Sec. 5.2, and then connected to the gradients of magnon chemical potential and temperature via the Boltzmann equations (see Sec. 5.3, where the drag resistivities are defined). In Sec. 5.6, we analyze the temperature dependence of magnon drag resistivities for fixed number of pumped magnons and for fixed magnon chemical potential. The dependence of layer distance is discussed in Sec. 5.7. Finally, we give some estimation on the magnitude of induced fields in the passive layer. The amplitude of interlayer dipolar interaction and the imaginary part of magnon response functions are discussed in detail in the appendices.

\subsection{Quantization of Ferromagnetic Spin Waves}

In previous studies, we treated spin-wave in ferromagnets semiclassically and solved its dispersion and wave functions under magneto-electric effect and different geometrical structures. Now, we change our gear to magneto-thermal effect and use the language of second quantization, i.e. treating spin waves as magnons, which obey Bose-Einstein 


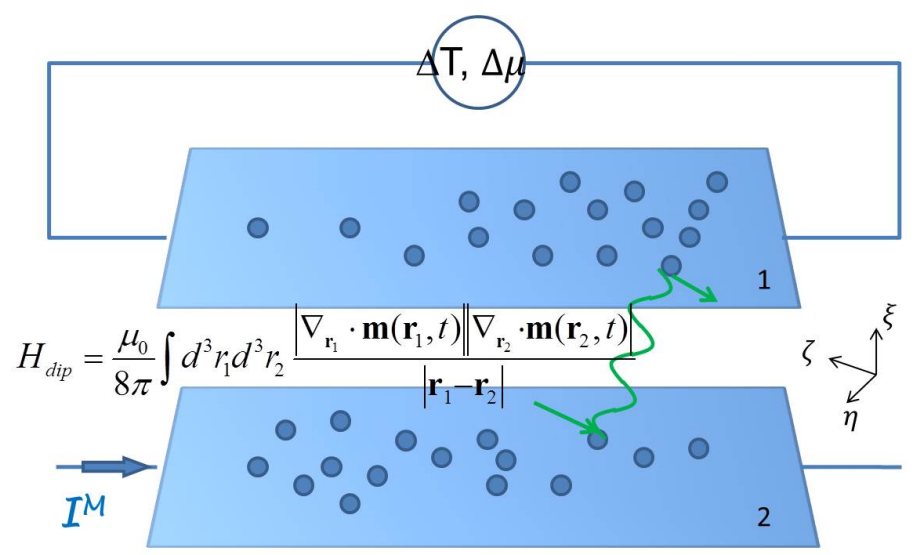

Figure 5.1: Schematic illustration of spin-wave drag. The blue dots represent magnons. The magnetization of the two layers are assumed to be in plane and parallel to each other.

statistics.

\subsubsection{Heisenberg Hamiltonian for Spin-1/2}

Let us start from a ferromagnet with all spins of magnitude $1 / 2$. This will help us to understand why spin-wave is a low lying collective excitation, as well as the physical meaning of spin-wave quantum.

$$
\begin{aligned}
H & =-J \sum_{i}^{N} \sum_{\delta}^{\nu} \mathbf{S}_{i} \mathbf{S}_{i+\delta} \\
& =-J \sum_{i}^{N} \sum_{\delta}^{\nu} \frac{1}{2}\left[S_{i}^{+} S_{i+\delta}^{-}+S_{i}^{-} S_{i+\delta}^{+}\right]+S_{i}^{z} S_{i+\delta}^{z},
\end{aligned}
$$

where $J$ is the exchange coefficient, $N$ is the number of spins and $\nu$ is the number of nearest neighboring spins. For a ferromagnet, $J>0$, the ground state should be all spins parallel to each other. Supposing all spins direct along $z$ axis, we denote 


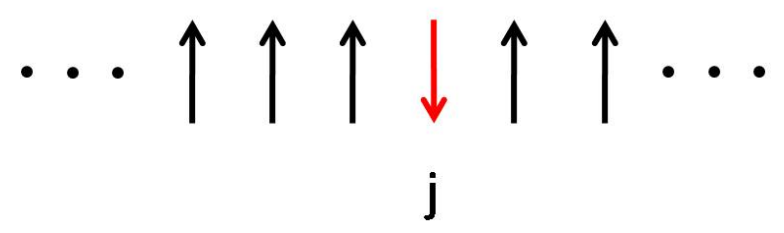

Figure 5.2: A single local spin-flip excitation of a ferromagnetic system. The resulting state is not an eigenstate of the Heisenberg Hamiltonian.

the ground sate as $|G\rangle=\prod_{i}|\uparrow\rangle_{i}$, with ground state energy $E_{0}=-\frac{1}{4} J \nu N$. At zero temperature, the ferromagnet is in its ground state. When $T \neq 0$, spins may start flipping due to thermal fluctuation. Consider a spin-flip excitation as shown in Fig. 5.2, denoted as $|\downarrow\rangle_{j}=S_{j}^{-}|G\rangle$. Because

$$
S_{j}^{+} S_{j+1}^{-}\left|\downarrow_{j} \uparrow_{j+1}\right\rangle=\left|\uparrow_{j} \downarrow_{j+1}\right\rangle
$$

a single local spin-flip excitation is not an eigenstate of the Heisenberg Hamiltonian. However, a linear combination of all these single flip states is an eigenstate. For example, let

$$
|k\rangle=\frac{1}{\sqrt{N}} \sum_{j} e^{i \mathbf{k} \cdot \mathbf{r}_{j}}|\downarrow\rangle_{j} .
$$

It is a proof that $|k\rangle$ is an eigenstate with eigen energy $E_{k}=E_{0}+J \nu\left[1-\frac{1}{\nu} \sum_{\delta} \cos (\mathbf{k}\right.$. $\left.\mathbf{r}_{\delta}\right)$ ]. The state $|k\rangle$ is called a spin-wave (or "magnon") of wave vector $\mathbf{k}$ and energy $E_{k}$. What is the physical meaning of state $|k\rangle$ ? Now define $S_{z} \equiv \sum_{i} S_{i}^{z}$, thus

$$
\begin{aligned}
S^{z}|k\rangle & =\sum_{i} S_{i}^{z} \frac{1}{\sqrt{N}} \sum_{j} e^{i \mathbf{k} \cdot \mathbf{r}_{j}}|\downarrow\rangle_{j} \\
& =\frac{1}{\sqrt{N}} \sum_{j} e^{i \mathbf{k} \cdot \mathbf{r}_{j}} \sum_{i} S_{i}^{z}|\downarrow\rangle_{j} .
\end{aligned}
$$


Noting that

$$
\begin{aligned}
S_{i}^{z}|\downarrow\rangle_{j} & =S|\downarrow\rangle_{j} \quad \text { if } i \neq j \\
& =(S-1)|\downarrow\rangle_{j} \quad \text { if } i=j,
\end{aligned}
$$

we get

$$
\begin{aligned}
S^{z}|k\rangle & =\frac{1}{\sqrt{N}} \sum_{j} e^{i \mathbf{k} \cdot \mathbf{r}_{j}}(N S-1)|\downarrow\rangle_{j} \\
& =(N S-1)|k\rangle
\end{aligned}
$$

with $S$ being the magnitude of local spin, that is, the average effect of $|k\rangle$ is the ground state with one spin flipped but it is in a way of collective motion instead of a local spin flip. Notice that at $k \rightarrow 0, E_{k}=E_{0}$, i.e., all the spins rotating as a whole does not consume energy.

\subsubsection{Second Quantization}

In Sec. 5.1.1, we discuss the case of spin-1/2. Now, we generalize the discussion to the case of local spins saturated with S. Suppose in ground state all spins are up and use a new notation $|0\rangle$ to denote the ground state since there is no spin waves. Then

we can use introduce the creation $\left(\hat{a}_{i}^{\dagger}\right)$ and annihilation $\left(\hat{a}_{i}\right)$ operators to describe the local spin-flip processes $S_{i}^{-}$and $S_{i}^{+}\left(\right.$since $\left.S_{i}^{+}|0\rangle=0\right)$. Now excite a spin-wave, $S^{z}|1\rangle=(S N-1)|1\rangle$; add another spin-wave, $\left\langle S^{z}\right\rangle=S N-2$; etc.. Therefore, 
$S_{i}^{z}=S-\hat{a}_{i}^{\dagger} \hat{a}_{i} ;$ together with

$$
S_{i}^{+}=\sqrt{2 S-\hat{n}_{i}} \hat{a}_{i} \text { and } S_{i}^{-}=\hat{a}_{i}^{\dagger} \sqrt{2 S-\hat{n}_{i}}
$$

where $\hat{n}_{i}=\hat{a}_{i}^{\dagger} \hat{a}_{i}$ with $\left\langle\hat{n}_{i}\right\rangle \leq 2 S$ and $\hat{a}_{i}$ and $\hat{a}_{i}^{\dagger}$ being Boson operators. These are the so called Holstein-Primakoff transformation [31]. The coefficients before $\hat{a}_{i}$ and $\hat{a}_{i}^{\dagger}$ ensure the commutations among $S_{i}^{+}, S_{i}^{-}$and $S_{i}^{z}$. For $S, N \gg 1$, it is reasonable to use the following approximations:

(i) $\sqrt{1-\frac{\hat{n}_{i}}{2 S}} \approx 1$;

(ii) $\hat{n}_{i} \hat{n}_{j}$ can be neglected.

The reasons for these are:

(i) $\left\langle\hat{n}_{i}\right\rangle=\left(\frac{1}{\sqrt{N}}\right)^{2} \sum_{j, j^{\prime}} e^{-i \mathbf{k} \cdot\left(\mathbf{r}_{j^{\prime}}-\mathbf{r}_{j}\right)}\left\langle\downarrow_{j^{\prime}}\left|\hat{n}_{i}\right| \downarrow_{j}\right\rangle=\frac{1}{N} \ll 2 S$;

(ii) $\left\langle\hat{n}_{i} \hat{n}_{j}\right\rangle \leq\left\langle\hat{n}_{i}\right\rangle\left\langle\hat{n}_{j}\right\rangle=\frac{1}{N^{2}} \ll 2 S$.

Therefore,

$$
S_{i}^{+}=\sqrt{2 S} \hat{a}_{i} \text { and } S_{i}^{-}=\sqrt{2 S} \hat{a}_{i}^{\dagger}
$$

notice that $\mathbf{M}=-\frac{g \mu_{B}}{\Omega} \mathbf{S}$, we get

$$
M_{i}^{+}=-\sqrt{\frac{2 g \mu_{B} M_{s}}{\Omega}} \hat{a}_{i} \text { and } M_{i}^{-}=-\sqrt{\frac{2 g \mu_{B} M_{s}}{\Omega}} \hat{a}_{i}^{\dagger}
$$

where $\mu_{B}=\frac{e h}{2 m}$ is the Bohr magnon, $M_{s}$ is the saturated magnetization and $\Omega$ is the volume of the unit cell. 


\subsection{Thermodynamic Definitions for magnon cur- rent density and heat current density}

Magnons are bosons whose equilibrium distribution satisfies Bose-Einstein statistics, that is

$$
n^{0}(\mathbf{k})=\frac{1}{e^{\frac{\epsilon_{k}-\mu}{k_{B} T}}-1},
$$

where $\epsilon_{k}=D k^{2}+\epsilon_{0}$ is the dispersion of magnons (neglect anisotropy in short wavelength limit, see App. B.2) and $k_{B}$ is the Boltzmann constant. It is worth noting that the chemical potential of magnons should be zero at a thermal equilibrium state, since the particle number is not conserved. However, because magnon-magnon relaxation time $(100-200 \mathrm{~ns})$ is much less than the magnon-lattice relaxation time $(1 \mu \mathrm{s})$, one can achieve a quasi-equilibrium state with a non-zero chemical potential by a thermodynamic process - the injection of additional magnons to the system that compensate the magnon decay. This is proved by the observation of magnon BEC at room temperature[32], where the finite chemical potential is controlled by the power and frequency of parametric pumping.

A schematic picture of the magnon drag measurement is shown in Fig. 5.1. The two layers are well separated in the sense of being thermally isolated and only magnetic dipolar interaction existing between the two layers. In analogy to Coulomb drag, we need a steady magnon current in the active layer (layer 2) and keep the passive layer (layer 1) in equilibrium (i.e., there is neither magnon current nor heat current). The magnon current density is defined in terms of magnon distribution function by

$$
\mathbf{j}=\sum_{\mathbf{k}} v_{\mathbf{k}} n(\mathbf{k})=\sum_{\mathbf{k}} v_{\mathbf{k}}\left[n^{0}(k)+\delta n(\mathbf{k})\right]
$$


where $\mathbf{v}_{k}=\frac{\partial \epsilon_{k}}{\partial \mathbf{k}}$ is the group velocity of magnons, and

$$
\delta n(\mathbf{k})=\frac{\partial n^{0}}{\partial \epsilon_{k}} \mathbf{v}_{k} \cdot\left(\mathbf{P}+\beta \tilde{\epsilon}_{k} \mathbf{P}_{T}\right)
$$

where $\beta=\frac{1}{k_{B} T}, \tilde{\epsilon}_{k} \equiv \epsilon_{k}-\mu, \mathbf{P}$ and $\mathbf{P}_{T}$ are the shifts of momentum that corresponds to the gradient of magnon chemical potential and the gradient of temperature, respectively. Since the quasi-equilibrium distribution does not depend on the direction of the wave vector as shown in Eq. (5.10), only the distribution out-of-equilibrium contributes to the current, that is

$$
\mathbf{j}=\sum_{\mathbf{k}} v_{\mathbf{k}} \delta n(\mathbf{k})=\sum_{\mathbf{k}} \mathbf{v}_{k} \frac{\partial n^{0}}{\partial \epsilon_{k}} \mathbf{v}_{k} \cdot\left(\mathbf{P}+\beta \tilde{\epsilon}_{k} \mathbf{P}_{T}\right)
$$

Similarly, one can define the magnon heat current density as

$$
\mathbf{j}_{Q}=\sum_{\mathbf{k}} \tilde{\epsilon}_{k} v_{\mathbf{k}} \delta n(\mathbf{k})=\sum_{\mathbf{k}} \tilde{\epsilon}_{k} \mathbf{v}_{k} \frac{\partial n^{0}}{\partial \epsilon_{k}} \mathbf{v}_{k} \cdot\left(\mathbf{P}+\beta \tilde{\epsilon}_{k} \mathbf{P}_{T}\right)
$$

For isotropic 2D magnon gas (short wavelength limit), the tensors involved $\mathbf{v}_{k} \mathbf{v}_{k}$ become diagonal, and we can get a simple relation between $\left(\mathbf{j}, \mathbf{j}_{Q}\right)$ and $\left(\mathbf{P}, \mathbf{P}_{T}\right)$, that is

$$
\left(\begin{array}{c}
\mathbf{j} \\
\mathbf{j}_{Q}
\end{array}\right)=\left(\begin{array}{ll}
A_{\mu \mu} & A_{\mu T} \\
A_{T \mu} & A_{T T}
\end{array}\right)\left(\begin{array}{c}
\mathbf{P} \\
\beta \mathbf{P}_{T}
\end{array}\right)
$$

In the limit of $\epsilon_{0}-\mu \ll k_{B} T$,

$$
\begin{aligned}
& A_{\mu \mu}=-\frac{2 n D}{\hbar^{2}}, \quad A_{\mu T}=A_{T \mu}=-\frac{\pi}{6 \hbar^{2} \beta^{2}} \\
& A_{T T}=-\frac{3 \zeta[3]}{\pi \hbar^{2} \beta^{3}}
\end{aligned}
$$


where $n$ is the density of $2 \mathrm{D}$ magnon gas, $D$ is the exchange stiffness, $\epsilon_{0}$ is the energy gap of magnon dispersion, and $\zeta$ is the Riemann zeta function.

\subsection{Drag Resistivities}

Before switching on the steady current, both layers are in thermal equilibrium at the same temperature $T$. A steady current in layer 2 brings its own distribution outof-equilibrium and drives layer 1 to a new equilibrium state through the inter-layer dipolar interaction. The change of distribution function in layer 1 can be obtained by using Boltzmann equation,

$$
\frac{\partial n_{1}}{\partial t}+\dot{\mathbf{k}} \nabla_{\mathbf{k}} n_{1}+\mathbf{v}_{k} \nabla_{\mathbf{r}} n_{1}=\left(\frac{\partial n_{1}(\mathbf{r}, \mathbf{k}, t)}{\partial t}\right)_{\text {coll12 }},
$$

where the dot on the top denotes the time derivative. In stationary case, $n_{1}$ does not

depend on time explicitly, i.e. $\frac{\partial n_{1}}{\partial t}=0$. And there is no confinement potential for the magnons within the plane, thus the second term on the r.h.s. of Eq. (5.17) is also zero. Suppose the newly equilibrium state does not deviate very much from the initial one, Eq. (5.17) becomes

$$
\left(-\frac{\partial n_{1}^{0}}{\partial \epsilon_{k}}\right) \mathbf{v}_{\mathbf{k}} \cdot\left(\boldsymbol{\nabla} \mu_{1}+\frac{\tilde{\epsilon}_{k}}{T} \boldsymbol{\nabla} T_{1}\right)=\left(\frac{\partial n_{1}(\mathbf{r}, \mathbf{k}, t)}{\partial t}\right)_{\text {coll } 12} .
$$

Define $\bar{n}_{\mathbf{k}}=1+n_{\mathbf{k}}$. The right hand side of Eq. (5.18) can be obtained by applying Fermi's golden rule to the dipolar interaction. Terms related to the outgoing processes 
have been shown in Fig. 5.3.

$$
\begin{aligned}
\left(\frac{\partial n_{1}}{\partial t}\right)_{\text {coll } 12}= & -\sum_{\mathbf{p}} \frac{2 \pi}{\hbar}|W(\mathbf{k})|^{2}\left(n_{1 \mathbf{k}} n_{2 \mathbf{p}} \bar{n}_{2, \mathbf{p}+\mathbf{k}}-\bar{n}_{1 \mathbf{k}} \bar{n}_{2 \mathbf{p}} n_{2, \mathbf{p}+\mathbf{k}}\right) \delta\left(\epsilon_{1 k}+\epsilon_{2 p}-\epsilon_{2, \mathbf{p}+\mathbf{k}}\right) \\
- & \sum_{\mathbf{p}} \frac{2 \pi}{\hbar}|W(\mathbf{p})|^{2}\left[\left(n_{1 \mathbf{k}} \bar{n}_{1, \mathbf{k}+\mathbf{p}} n_{2 \mathbf{p}}-\bar{n}_{1 \mathbf{k}} n_{1, \mathbf{k}+\mathbf{p}} \bar{n}_{2, \mathbf{p}}\right) \delta\left(\epsilon_{1 k}+\epsilon_{2 p}-\epsilon_{1, \mathbf{k}+\mathbf{p}}\right)\right. \\
& +\left(n_{1 \mathbf{k}} \bar{n}_{1, \mathbf{k}+\mathbf{p}} \bar{n}_{2,-\mathbf{p}}-\bar{n}_{1 \mathbf{k}} n_{1, \mathbf{k}+\mathbf{p}} n_{2,-\mathbf{p}}\right) \delta\left(\epsilon_{1 k}-\epsilon_{2 p}-\epsilon_{1, \mathbf{k}+\mathbf{p})]}\right)
\end{aligned}
$$

with

$$
W(\mathbf{k})=\frac{\sqrt{2}}{8} \mu_{0}\left(\frac{g \mu_{B}}{L}\right)^{\frac{3}{2}} \frac{\sqrt{M_{s}}}{k} e^{-k(d-L)}\left(1-e^{-k L}\right)^{2}\left(\cos \varphi_{\mathbf{k}}+\sin \varphi_{\mathbf{k}} \cos \varphi_{\mathbf{k}}\right),
$$

where $d$ is the distance between the two layers, $L$ is the thickness of each layer and $\varphi_{\mathbf{k}}$ is the angle between $\mathbf{k}$ and $-\mathbf{M}_{s} . W(\mathbf{k})$ is obtained from the interlayer dipolar interaction,

$$
\mathscr{H}_{d}=\frac{\mu_{0}}{2} \int_{d-\frac{L}{2}}^{d+\frac{L}{2}} d \xi \int d \mathbf{r} \int_{\frac{L}{2}}^{\frac{L}{2}} d \xi^{\prime} \int d \mathbf{r}^{\prime} \frac{\left[\nabla_{\mathbf{r}} \cdot \mathbf{m}(\mathbf{r}, \xi)\right]\left[\nabla_{\mathbf{r}^{\prime}} \cdot \mathbf{m}\left(\mathbf{r}^{\prime}, \xi^{\prime}\right)\right]}{\sqrt{\left|\mathbf{r}-\mathbf{r}^{\prime}\right|^{2}+\left(\xi-\xi^{\prime}\right)^{2}}}
$$

by doing the Holstein-Primakoff transformation,

$$
\begin{aligned}
m^{x}(\mathbf{r}, \xi) & =\frac{1}{2} \sqrt{\frac{2 \hbar \gamma M_{s}}{V}} \sum_{n, \mathbf{k}}\left(a_{n \mathbf{k}}+a_{n,-\mathbf{k}}^{*}\right) \Phi_{n}(\xi) e^{i \mathbf{k} \cdot \mathbf{r}}, \\
m^{y}(\mathbf{r}, \xi) & =\frac{1}{2 i} \sqrt{\frac{2 \hbar \gamma M_{s}}{V}} \sum_{n, \mathbf{k}}\left(a_{n \mathbf{k}}-a_{n,-\mathbf{k}}^{*}\right) \Phi_{n}(\xi) e^{i \mathbf{k} \cdot \mathbf{r}}, \\
m^{z}(\mathbf{r}, \xi) & =M_{s}-\frac{\hbar \gamma}{V} \sum_{m, \mathbf{k}} \sum_{n, \mathbf{k}^{\prime}} a_{m \mathbf{k}}^{*} a_{n \mathbf{k}^{\prime}} \Phi_{m}(\xi) \Phi_{n}(\xi) e^{i\left(\mathbf{k}^{\prime}-\mathbf{k}\right) \cdot \mathbf{r}},
\end{aligned}
$$


where we have chosen the equilibrium magnetization to be along $z$-axis, $\mathbf{r}$ is the position vector within the film plane, $\xi$ is the coordinate perpendicular to the film, $\gamma=$ $g \mu_{B} / \hbar$ is the gyromagnetic ratio, $M_{s}$ is the saturation magnetization, $V$ is the volume of the film, $m$ and $n$ denote the different thickness bands and the orthogonal functions $\Phi_{n}(\xi)$ are real and given in the appendix. Assuming the two layers have unpinned boundary and keeping only the lowest transverse band $(n=0)$ that contributes the most in thin film structure, we arrive at

$$
\begin{aligned}
\mathscr{H}_{d}= & \frac{\sqrt{2}}{4} \mu_{0}\left(\frac{\hbar \gamma}{V}\right)^{\frac{3}{2}} \sqrt{M_{s}} \sum_{\mathbf{k} \mathbf{p}}\left[a_{\mathbf{k}} b_{\mathbf{p}+\mathbf{k}}^{*} b_{\mathbf{p}} i\left(\frac{1}{2} P(k) \sin 2 \varphi_{\mathbf{k}}+Q(k) \cos \varphi_{\mathbf{k}}\right)\right. \\
& \left.+a_{\mathbf{k}+\mathbf{p}}^{*} a_{\mathbf{k}} b_{\mathbf{p}} i\left(\frac{1}{2} P(p) \sin 2 \varphi_{\mathbf{p}}+Q(p) \cos \varphi_{\mathbf{p}}\right)+c . c .\right]
\end{aligned}
$$

where $a\left(a^{*}\right)$ and $\mathrm{b}\left(b^{*}\right)$ denote the amplitudes of spin-wave mode in layer 1 and layer 2 , respectively. Besides of the three-magnon interaction, there are also four-magnon interactions as shown in App. B. Generally speaking, four-magnon processes fall into two categories (Fig. 5.4): three magnons from one layer interacting with one magnon from the other layer, and the Coulomb-like interaction. It turns out that drag resistivities contributed by four-magnon interactions is only $0.1 \%$ of that due to three-magnon interaction because of an extra power of the small parameter $\frac{g \mu_{B}}{L a^{2} M_{s}}$, with $a$ being the lattice constants. Therefore, we drop the interactions more than three magnons here.

$$
P(k)=Q(k)=\frac{1}{2 k} e^{-k(d-L)}\left(1-e^{-k L}\right)^{2} .
$$


Eq. (5.25) indicates two processes labeled in A and B in Fig. 5.3.

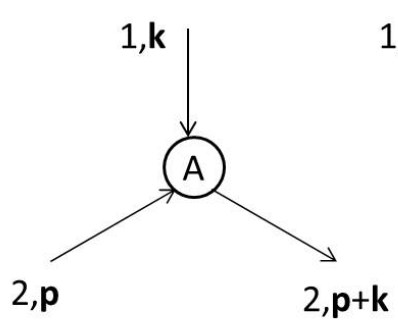

(a)

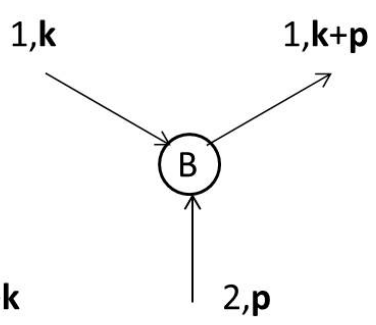

(b)

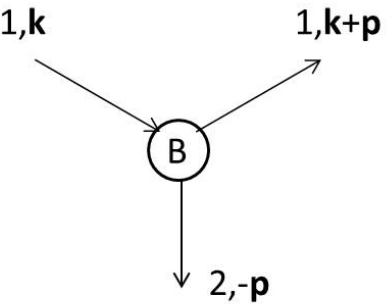

(c)

Figure 5.3: Interlayer three-magnon interactions. The figure shows the outgoing processes of magnons in layer 1.1 and 2 label the different layers. A and B correspond to the interaction amplitudes $W(\mathbf{k})$ and $W(\mathbf{p})$, respectively.

The details of how to get the amplitude $W(\mathbf{k})$ is shown in App. B. It is worth noting that the tunneling of magnons between the two layers has been suppressed by requiring that the magnons in the two layers have slightly different dispersions. (For example, the two layers are subject to different Zeeman fields or they are made of materials of different exchange stiffness.) Such a requirement breaks the energy conservation law obeyed by the interlayer two-magnon interaction and therefore forbids the "tunneling" process.

The Boltzmann equation for layer 2 can be obtained by interchanging subscripts 1 and $2, \mathbf{k}$ and $\mathbf{p}$ in Eq. (5.18). We need four equations to build the connection between $\left(\mathbf{P}_{1}, \beta_{1} \mathbf{P}_{T 1}, \mathbf{P}_{2}, \beta_{2} \mathbf{P}_{T 2}\right)$ and $\left(\boldsymbol{\nabla} \mu_{1}, \boldsymbol{\nabla} T_{1}, \boldsymbol{\nabla} \mu_{2}, \boldsymbol{\nabla} T_{2}\right)$. Two of these equations are obtained by: first, multiplying both sides of Eq. (5.18) by $\mathbf{k}$ and doing the summation over $\mathbf{k}$; then, multiplying both sides of Eq. (5.18) by $\tilde{\epsilon}_{1 k} \mathbf{k}$ and doing the summation over $\mathbf{k}$. These two manipulations correspond to the calculation of rate of change of momentum and rate of change of heat current, respectively. Doing 


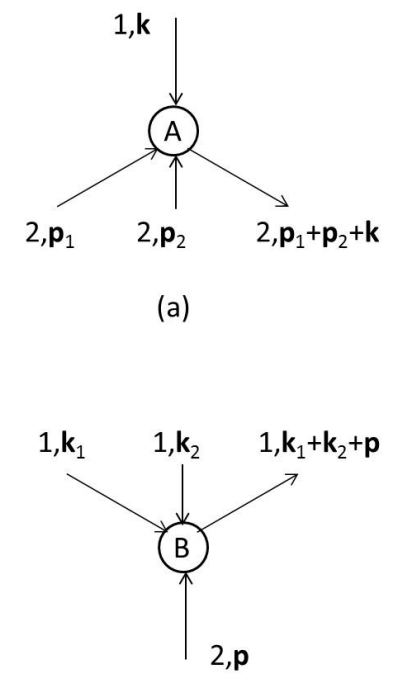

(d)

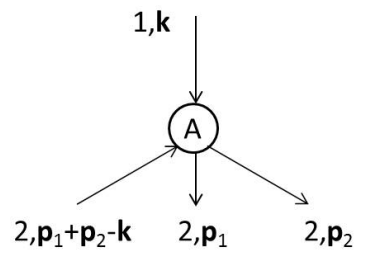

(b)

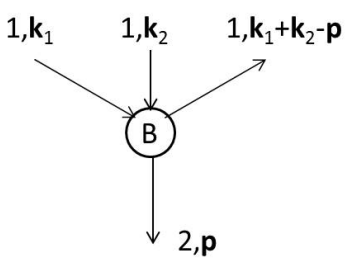

(e)

[Interlayer four-magnon interactions.]

Figure 5.4: Interlayer four-magnon interactions. The figure shows the outgoing processes of magnons in layer 1: 1 and 2 label the different layers; A and B correspond to the interaction amplitudes $W(\mathbf{k})$ and $W(\mathbf{p})$, respectively; (f) illustrates the Coulomblike interaction where $W_{q}$ emphasizes that the transition amplitude depends on the momentum transfer only.

similar transformations to the Boltzmann equation in layer 2, we obtain the other two equations.

Due to the angular-dependence of the interlayer interaction, we come across the integral of the following form when calculating the rate of change of momentum

$$
\sum_{\mathbf{k}} \hat{\mathbf{k}}\left(\hat{\mathbf{k}} \cdot \mathbf{P}_{i}\right) f(k)(\cos \varphi+\sin \varphi \cos \varphi)^{2}
$$

whose direction is determined by $\mathbf{k}$. $f$ is a function that depends on the magnitude of $\mathbf{k}$. As shown in Fig. 5.5, $\mathbf{P}_{i}$ can direct arbitrary direction. 


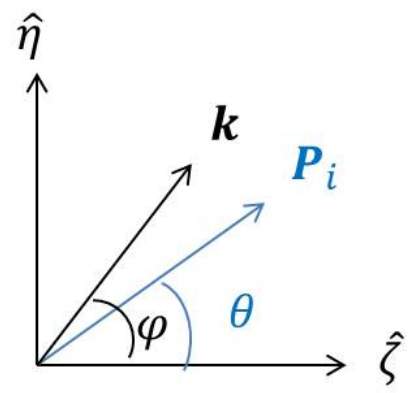

Figure 5.5: Relative direction of $\mathbf{P}_{i}$ and $\mathbf{k} . \hat{\boldsymbol{\zeta}}$ is along $-\mathbf{M}_{s}$.

$$
\begin{aligned}
\hat{\mathbf{k}}\left(\hat{\mathbf{k}} \cdot \mathbf{P}_{i}\right)= & \hat{\boldsymbol{\zeta}}\left(k^{2} \cos ^{2} \varphi P_{i, \zeta}+k^{2} \cos \varphi \sin \varphi P_{i, \eta}\right) \\
& +\hat{\boldsymbol{\eta}}\left(k^{2} \cos \varphi \sin \varphi P_{i, \zeta}+k^{2} \sin ^{2} \varphi P_{i, \eta}\right)
\end{aligned}
$$

Since

$$
\begin{aligned}
\frac{1}{2 \pi} \int_{0}^{2 \pi} \cos ^{2} \varphi(\cos \varphi+\sin \varphi \cos \varphi)^{2} d \varphi & =\frac{7}{16} \\
\frac{1}{2 \pi} \int_{0}^{2 \pi} \sin ^{2} \varphi(\cos \varphi+\sin \varphi \cos \varphi)^{2} d \varphi & =\frac{3}{16} \\
\frac{1}{2 \pi} \int_{0}^{2 \pi} \cos \varphi \sin \varphi(\cos \varphi+\sin \varphi \cos \varphi)^{2} d \varphi & =0,
\end{aligned}
$$

Eq. (5.27) is equivalent to $\sum_{k} \frac{7 P_{i, \zeta} \hat{\boldsymbol{\zeta}}+3 P_{i, \eta} \hat{\boldsymbol{\eta}}}{16}(\hat{\mathbf{k}} \cdot \hat{\mathbf{k}}) f(k)$. 
As a result, we get

$$
\begin{aligned}
& -\left(\begin{array}{cccc}
A_{\mu \mu}^{(1)} & A_{\mu T}^{(1)} & 0 & 0 \\
A_{T \mu}^{(1)} & A_{T T}^{(1)} & 0 & 0 \\
0 & 0 & A_{\mu \mu}^{(2)} & A_{\mu T}^{(2)} \\
0 & 0 & A_{T \mu}^{(2)} & A_{T T}^{(2)}
\end{array}\right)\left(\begin{array}{c}
\boldsymbol{\nabla} \mu_{1} \\
\boldsymbol{\nabla}_{T_{1}} \\
T_{1} \\
\nabla \mu_{2} \\
\nabla_{T_{2}}
\end{array}\right) \\
= & \frac{2 D}{\hbar}\left(\begin{array}{cccc}
B_{\mu \mu}^{11} & B_{\mu T}^{11} & B_{\mu \mu}^{12} & B_{\mu T}^{12} \\
B_{T \mu}^{11} & B_{T T}^{11} & B_{T \mu}^{12} & B_{T T}^{12} \\
B_{\mu \mu}^{21} & B_{\mu T}^{21} & B_{\mu \mu}^{22} & B_{\mu T}^{22} \\
B_{T \mu}^{21} & B_{T T}^{21} & B_{T \mu}^{22} & B_{T T}^{22}
\end{array}\right)\left(\begin{array}{c}
P_{1} \\
\beta_{1} P_{T 1} \\
P_{2} \\
\beta_{2} P_{T 2}
\end{array}\right) \boldsymbol{\theta},
\end{aligned}
$$

where $\boldsymbol{\theta}=\frac{7 \cos \theta \hat{\boldsymbol{\zeta}}+3 \sin \theta \hat{\boldsymbol{\eta}}}{16}$ denotes the direction of the induced fields. $\hat{\boldsymbol{\zeta}}$ (anti-parallel to $\left.\mathbf{M}_{s}\right)$ and $\hat{\boldsymbol{\eta}}$ are in-plane, $\zeta-\eta-\xi$ forms the right-hand reference frame as shown in Fig. 5.1 and $\theta$ is the angle between the driving current and the saturation magnetization as shown in Fig. 5.5. Before go to the detail of what the matrix elements are, let us introduce some symbols to see the structure of the thermoelectric properties under consideration. Let

$$
\mathbf{F}_{i} \equiv\left(\begin{array}{c}
\nabla \mu_{i} \\
\frac{\nabla_{T_{i}}}{T_{i}}
\end{array}\right), \quad \mathbf{J}_{i} \equiv\left(\begin{array}{c}
\mathbf{j}_{i} \\
\mathbf{j}_{Q i}
\end{array}\right), \quad \mathbf{A}^{(i)} \equiv\left(\begin{array}{cc}
A_{\mu \mu}^{(i)} & A_{\mu T}^{(i)} \\
A_{T \mu}^{(i)} & A_{T T}^{(i)}
\end{array}\right), \quad \mathbf{B}^{i j} \equiv\left(\begin{array}{cc}
B_{\mu \mu}^{i j} & B_{\mu T}^{i j} \\
B_{T \mu}^{i j} & B_{T T}^{i j}
\end{array}\right),
$$

with $i, j=1,2$. From Eq. (5.15) and (5.29), we find the relation between the current 
densities and the external fields,

$$
\left(\begin{array}{c}
\mathbf{F}_{1} \\
\mathbf{F}_{2}
\end{array}\right)=-\left(\begin{array}{cc}
\mathbf{A}^{(1)} & 0 \\
0 & \mathbf{A}^{(2)}
\end{array}\right)^{-1} \frac{2 D}{\hbar}\left(\begin{array}{ll}
\mathbf{B}^{11} & \mathbf{B}^{12} \\
\mathbf{B}^{21} & \mathbf{B}^{22}
\end{array}\right)\left(\begin{array}{cc}
\mathbf{A}^{(1)} & 0 \\
0 & \mathbf{A}^{(2)}
\end{array}\right)^{-1}\left(\begin{array}{l}
J_{1} \\
J_{2}
\end{array}\right) \boldsymbol{\theta}
$$

Suppose the two layers are set up as follows. In one layer ("active layer") there flows the magnon current, and in the other layer ("passive layer") there are induced fields to compensate the currents. If we choose layer 1 as the passive layer and layer 2 the active one, there exists the relation

$$
\left(\begin{array}{c}
\boldsymbol{\nabla} \mu_{1} \\
\frac{\nabla_{T_{1}}}{T_{1}}
\end{array}\right)=-\frac{2 D}{\hbar}\left(\mathbf{A}^{(1)}\right)^{-1} \mathbf{B}^{12}\left(\mathbf{A}^{(2)}\right)^{-1}\left(\begin{array}{c}
j_{2} \\
j_{Q 2}
\end{array}\right) \boldsymbol{\theta}
$$

Therefore, we define $\mathbf{C}=\frac{2 D}{\hbar}\left(\mathbf{A}^{(1)}\right)^{-1} \mathbf{B}^{12}\left(\mathbf{A}^{(2)}\right)^{-1} \boldsymbol{\theta}$ whose elements corresponds to the thermal/spin drag resistivities. The relative direction of the induced fields and the driving current is shown in Fig. 5.6. It shows that the induced field is maximum when the driving current is parallel to the saturation magnetization and minimum when the two are perpendicular.

\subsection{Rate of Change of Momentum}

In this section, we discuss the rate of change of momentum owing to the interlayer dipolar interaction. It leads to the matrix elements of $B_{\mu \alpha}^{i j}$ with $\alpha=\mu, T$ and 


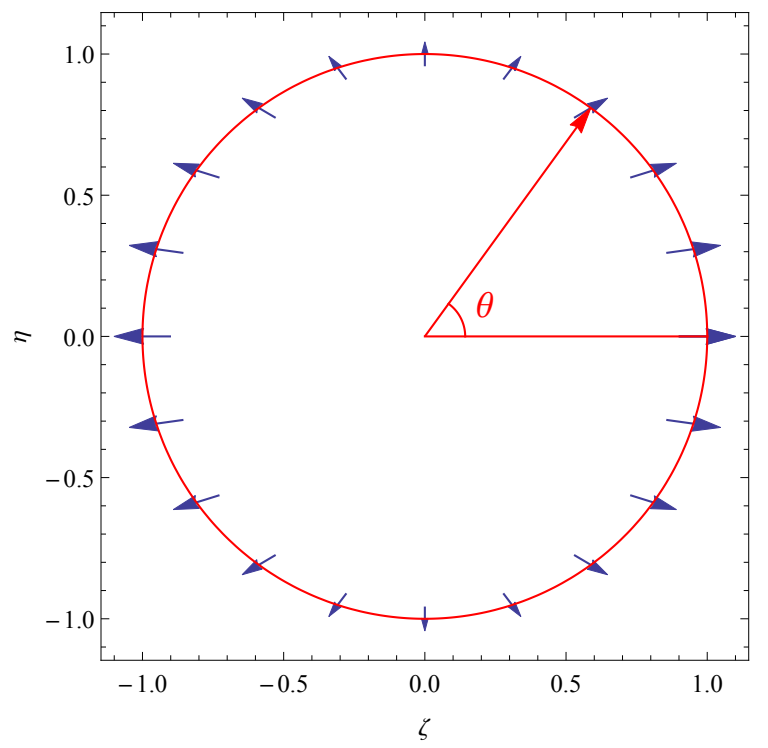

Figure 5.6: Relative direction of induced fields and driving current. The red arrow shows the direction of the driving current, which is always along the radius of the red ring. $\hat{\boldsymbol{\zeta}}$ is along $-\mathbf{M}_{s}$. The blue arrows show the direction and relative magnitude of induced field for a given direction of the driving current. 
$i, j=1,2$. We define the interlayer collision integral $\left(\frac{\partial n_{1}}{\partial t}\right)_{\text {coll12 }}=I_{A}(\mathbf{k})+I_{B}(\mathbf{k})$ with

$$
\begin{aligned}
I_{A}(\mathbf{k})= & -\sum_{\mathbf{p}} \frac{2 \pi}{\hbar}\left|W_{1}(\mathbf{k})\right|^{2}\left(n_{1 \mathbf{k}} n_{2 \mathbf{p}} \bar{n}_{2, \mathbf{p}+\mathbf{k}}-\bar{n}_{1 \mathbf{k}} \bar{n}_{2 \mathbf{p}} n_{2, \mathbf{p}+\mathbf{k}}\right) \\
& \times \delta\left(\epsilon_{1 k}+\epsilon_{2 p}-\epsilon_{2, \mathbf{p}+\mathbf{k}}\right) \\
I_{B}(\mathbf{k})= & -\sum_{\mathbf{p}} \frac{2 \pi}{\hbar}\left|W_{2}(\mathbf{p})\right|^{2}\left[\left(n_{1 \mathbf{k}} \bar{n}_{1, \mathbf{k}+\mathbf{p}} n_{2 \mathbf{p}}-\bar{n}_{1 \mathbf{k}} n_{1, \mathbf{k}+\mathbf{p}} \bar{n}_{2, \mathbf{p}}\right) \delta\left(\epsilon_{1 k}+\epsilon_{2 p}-\epsilon_{1, \mathbf{k}+\mathbf{p}}\right)\right. \\
& +\left(n_{1 \mathbf{k}} \bar{n}_{1, \mathbf{k}+\mathbf{p}} \bar{n}_{2,-\mathbf{p}}-\bar{n}_{1 \mathbf{k}} n_{1, \mathbf{k}+\mathbf{p}} n_{2,-\mathbf{p}}\right) \delta\left(\epsilon_{1 k}-\epsilon_{2 p}-\epsilon_{1, \mathbf{k}+\mathbf{p})] .}\right.
\end{aligned}
$$

The schematic picture of interactions A and B has been shown in Fig. 5.3.

According to Eq. (5.12),

$$
n_{i \mathbf{k}}=n_{i k}^{0}+\frac{n_{i}^{0}}{\partial \mathbf{k}} \cdot\left(\mathbf{P}_{i}+\beta_{i} \tilde{\epsilon}_{i k} \mathbf{P}_{T i}\right)
$$

with $i=1,2$. Let $\mathbf{P}_{i \mathbf{k}}=\mathbf{P}_{i}+\beta_{i} \tilde{\epsilon}_{i k} \mathbf{P}_{T i}$. We linearize $I_{A, B}(\mathbf{k})$ about $\mathbf{P}_{i}$ and $\mathbf{P}_{T i}$ and 
use the shorthand notation $\mathbf{p}+=\mathbf{p}+\frac{\mathbf{k}}{2}$ and $\mathbf{p}-=\mathbf{p}-\frac{\mathbf{k}}{2}$. Then

$$
\begin{aligned}
I_{A}(\mathbf{k})= & -\frac{2 \pi}{\hbar} \sum_{\mathbf{p}, \omega}|W(\mathbf{k})|^{2} \\
& \times\left[\left(\frac{\mathbf{P}_{1 k}}{n_{1 k}^{0}} \cdot \frac{\partial n_{1 k}^{0}}{\partial \mathbf{k}}+\frac{\mathbf{P}_{2 \mathbf{p}-}}{n_{2 \mathbf{p}-}^{0}} \cdot \frac{\partial n_{2 \mathbf{p}-}^{0}}{\partial \mathbf{p}}+\frac{\mathbf{P}_{2 \mathbf{p}+}}{\bar{n}_{2 \mathbf{p}+}^{0}} \cdot \frac{\partial \bar{n}_{2 \mathbf{p}+}^{0}}{\partial \mathbf{p}}\right) n_{1 k}^{0} n_{2 \mathbf{p}-}^{0} \bar{n}_{2 \mathbf{p}+}^{0}\right. \\
& \left.-\left(\frac{\mathbf{P}_{1 k}}{\bar{n}_{1 k}^{0}} \cdot \frac{\partial \bar{n}_{1 k}^{0}}{\partial \mathbf{k}}+\frac{\mathbf{P}_{2 \mathbf{p}-}}{\bar{n}_{2 \mathbf{p}-}^{0}} \cdot \frac{\partial \bar{n}_{2 \mathbf{p}-}^{0}}{\partial \mathbf{p}}+\frac{\mathbf{P}_{2 \mathbf{p}+}}{n_{2 \mathbf{p}+}^{0}} \cdot \frac{\partial n_{2 \mathbf{p}+}^{0}}{\partial \mathbf{p}}\right) \bar{n}_{1 k}^{0} \bar{n}_{2 \mathbf{p}-}^{0} n_{2 \mathbf{p}+}^{0}\right] \\
& \times \delta\left(\epsilon_{1 k}-\omega\right) \delta_{2 \epsilon} \\
= & -\frac{2 \pi}{\hbar}|W(\mathbf{k})|^{2} \sum_{\mathbf{p}, \omega}\left\{\mathbf{P}_{1} \cdot \frac{\partial n_{1 k}^{0}}{\partial \mathbf{k}}\left(n_{2 \mathbf{p}-}^{0}-n_{2 \mathbf{p}+}^{0}\right)\right. \\
& +\mathbf{P}_{2} \cdot\left[n_{1 k}^{0} \frac{\partial}{\partial \mathbf{p}}\left(n_{2 \mathbf{p}-}^{0}-n_{2 \mathbf{p}+}^{0}\right)-\frac{\partial}{\partial \mathbf{p}}\left(\bar{n}_{2 \mathbf{p}-}^{0} n_{2 \mathbf{p}+}^{0}\right)\right] \\
& +\beta_{1} \mathbf{P}_{T 1} \cdot \tilde{\epsilon}_{1 k} \frac{\partial n_{1 k}^{0}}{\partial \mathbf{k}}\left(n_{2 \mathbf{p}-}^{0}-n_{2 \mathbf{p}+}^{0}\right) \\
& +\beta_{2} \mathbf{P}_{T 2} \cdot\left\{\left(D_{2} p^{2}+\frac{1}{4} D_{2} k^{2}+\omega+\epsilon_{02}-\mu_{2}\right)\right. \\
& \times\left[n_{1 k}^{0} \frac{\partial}{\partial \mathbf{p}}\left(n_{2 \mathbf{p}-}^{0}-n_{2 \mathbf{p}+}^{0}\right)-\frac{\partial}{\partial \mathbf{p}}\left(\bar{n}_{2 \mathbf{p}-}^{0} n_{2 \mathbf{p}+}^{0}\right)\right] \\
& \left.+\omega\left[n_{1 k}^{0} \frac{\partial}{\partial \mathbf{k}}\left(n_{2 \mathbf{p}-}^{0}-n_{2 \mathbf{p}+}^{0}\right)-\frac{\partial}{\partial \mathbf{k}}\left(\bar{n}_{2 \mathbf{p}-}^{0} n_{2 \mathbf{p}+}^{0}\right)\right]\right\} \delta\left(\epsilon_{1 k}-\omega\right) \delta_{2 \epsilon},
\end{aligned}
$$

where we have used $n_{B i}(\omega)=-\left[n_{B i}(-\omega)+1\right]$ and defined $\delta_{2 \epsilon} \equiv \delta\left(\epsilon_{2 \mathbf{p}-}-\epsilon_{2 \mathbf{p}+}+\omega\right)$. 
Similarly, the process B is linearized as

$$
\begin{aligned}
I_{B}(\mathbf{k})= & -\frac{2 \pi}{\hbar} \sum_{\mathbf{p}, \omega}|W(\mathbf{p})|^{2} \\
& \times\left\{\mathbf { P } _ { 1 } \cdot \left\{\left[n_{2 p}^{0} \frac{\partial}{\partial \mathbf{k}}\left(n_{1 \mathbf{k}-}^{0}-n_{1 \mathbf{k}+}^{0}\right)-\frac{\partial}{\partial \mathbf{k}}\left(\bar{n}_{1 \mathbf{k}-}^{0} n_{1 \mathbf{k}+}^{0}\right)\right] \delta\left(\epsilon_{2 p}-\omega\right) \delta_{1 \epsilon}\right.\right. \\
& \left.+\left[n_{2 p}^{0} \frac{\partial}{\partial \mathbf{k}}\left(n_{1 \mathbf{k}-}^{0}-n_{1 \mathbf{k}+}^{0}\right)+\frac{\partial}{\partial \mathbf{k}}\left(n_{1 \mathbf{k}-}^{0} \bar{n}_{1 \mathbf{k}+}^{0}\right)\right] \delta\left(\epsilon_{2 p}+\omega\right) \delta_{1 \epsilon}\right\} \\
& +\mathbf{P}_{2} \cdot \frac{\partial n_{2 p}^{0}}{\partial \mathbf{p}}\left(n_{1 \mathbf{k}-}^{0}-n_{1 \mathbf{k}+}^{0}\right)\left[\delta\left(\epsilon_{2 p}-\omega\right)-\delta\left(\epsilon_{2 p}+\omega\right)\right] \delta_{1 \epsilon} \\
& +\beta_{1} \mathbf{P}_{T 1} \cdot\left\{\left\{\left(D_{1} k^{2}+\frac{1}{4} D_{1} p^{2}+\omega+\epsilon_{01}-\mu_{1}\right)\right.\right. \\
& \times\left[n_{2 p}^{0} \frac{\partial}{\partial \mathbf{k}}\left(n_{1 \mathbf{k}-}^{0}-n_{1 \mathbf{k}+}^{0}\right)-\frac{\partial}{\partial \mathbf{k}}\left(\bar{n}_{1 \mathbf{k}-}^{0} n_{1 \mathbf{k}+}^{0}\right)\right] \\
& \left.+\omega\left[n_{2 p}^{0} \frac{\partial}{\partial \mathbf{p}}\left(n_{1 \mathbf{k}-}^{0}-n_{1 \mathbf{k}+}^{0}\right)-\frac{\partial}{\partial \mathbf{p}}\left(\bar{n}_{1 \mathbf{k}-}^{0} n_{1 \mathbf{k}+}^{0}\right)\right]\right\} \delta\left(\epsilon_{2 p}-\omega\right) \delta_{1 \epsilon} \\
& +\left\{\left(D_{1} k^{2}+\frac{1}{4} D_{1} p^{2}+\omega+\epsilon_{01}-\mu_{1}\right)\left[n_{2 p}^{0} \frac{\partial}{\partial \mathbf{k}}\left(n_{1 \mathbf{k}-}^{0}-n_{1 \mathbf{k}+}^{0}\right)+\frac{\partial}{\partial \mathbf{k}}\left(n_{1 \mathbf{k}-}^{0} \bar{n}_{1 \mathbf{k}+}^{0}\right)\right]\right. \\
& \left.\left.+\omega\left[n_{2 p}^{0} \frac{\partial}{\partial \mathbf{p}}\left(n_{1 \mathbf{k}-}^{0}-n_{1 \mathbf{k}+}^{0}\right)+\frac{\partial}{\partial \mathbf{p}}\left(n_{1 \mathbf{k}-}^{0} \bar{n}_{1 \mathbf{k}+}^{0}\right)\right]\right\} \delta\left(\epsilon_{2 p}+\omega\right) \delta_{1 \epsilon}\right\} \\
& \left.+\beta_{2} \mathbf{P}_{T 2} \cdot \tilde{\epsilon}_{2 p} \frac{\partial n_{2 p}^{0}}{\partial \mathbf{p}}\left(n_{1 \mathbf{k}-}^{0}-n_{1 \mathbf{k}+}^{0}\right)\left[\delta\left(\epsilon_{2 p}-\omega\right)-\delta\left(\epsilon_{2 p}+\omega\right)\right] \delta_{1 \epsilon}\right\}
\end{aligned}
$$

with $\delta_{1 \epsilon} \equiv \delta\left(\epsilon_{1 \mathbf{k}-}-\epsilon_{1 \mathbf{k}+}+\omega\right)$.

A couple of technical manipulations are needed to express the momentum transfer rate in terms of linear response functions. First,

$$
n_{1 \mathbf{p}+}^{0} \bar{n}_{1 \mathbf{p}^{-}}^{0}=\left(n_{1 \mathbf{p}^{-}}^{0}-n_{2 \mathbf{p}+}^{0}\right) n_{B 1}\left(\epsilon_{\mathbf{p}^{+}}-\epsilon_{\mathbf{p}^{-}}\right)
$$

with $n_{B i}(\epsilon)=1 /\left(e^{\beta_{i} \epsilon}-1\right)\left(i=1,2 ; \beta_{i}=\frac{1}{k_{B} T_{i}}\right)$. Second, some partial derivatives with 
respect to $\mathbf{p}$ can be transformed to the partial derivatives with respect to $\omega$ as below,

$$
\begin{aligned}
\sum_{\mathbf{p}} \mathbf{F}(\mathbf{p}) \cdot \frac{\partial}{\partial \mathbf{p}}[\delta(2 D \mathbf{p} \cdot \mathbf{k}-\omega)] & =\sum_{\mathbf{p}} \mathbf{F}(\mathbf{p}) \cdot(-2 D \mathbf{k}) \frac{\partial}{\partial \omega}[\delta(2 D \mathbf{p} \cdot \mathbf{k}-\omega)] \\
& =-\frac{\partial}{\partial \omega} \sum_{\mathbf{p}} \mathbf{F}(\mathbf{p}) \cdot 2 D \mathbf{k} \delta(2 D \mathbf{p} \cdot \mathbf{k}-\omega)
\end{aligned}
$$

Using the above manipulations and Eq. (5.36) and (5.37) and assuming $\mathbf{P}_{i}$ and $\mathbf{P}_{T i}$ to be parallel to $-\mathbf{M}_{s}$, we obtain

$$
\begin{aligned}
B_{\mu \mu}^{11}= & \frac{7 \beta_{1}}{16} \sum_{k, \omega} \frac{2 \pi}{\hbar}|W(k)|^{2} 2 D_{1} k^{2} n_{1 k}^{0} \bar{n}_{1 k}^{0} \delta\left(\epsilon_{1 k}-\omega\right) \frac{\chi_{2}^{\prime \prime}(k, \omega)}{-\pi} \\
& +\frac{7}{16} \sum_{p, \omega} \frac{2 \pi}{\hbar}|W(p)|^{2} 2 D_{1} p^{2} \delta\left(\epsilon_{2 p}-\omega\right) \\
& \times\left\{n_{2 p}^{0} \frac{\partial}{\partial \omega}\left[\frac{\chi_{1}^{\prime \prime}(p, \omega)}{-\pi}\right]-\frac{\partial}{\partial \omega}\left[\frac{\chi_{1}^{\prime \prime}(p, \omega)}{-\pi} n_{B 1}(\omega)\right]\right\}, \\
B_{\mu \mu}^{12}= & -\frac{7}{16} \sum_{k, \omega} \frac{2 \pi}{\hbar}|W(k)|^{2} 2 D_{2} k^{2} \delta\left(\epsilon_{1 k}-\omega\right) \\
& \times\left\{n_{1 k}^{0} \frac{\partial}{\partial \omega}\left[\frac{\chi_{2}^{\prime \prime}(k, \omega)}{-\pi}\right]-\frac{\partial}{\partial \omega}\left[\frac{\chi_{2}^{\prime \prime}(k, \omega)}{-\pi} n_{B 2}(\omega)\right]\right\} \\
& -\frac{7 \beta_{2}}{16} \sum_{p, \omega} \frac{2 \pi}{\hbar}|W(p)|^{2} 2 D_{2} p^{2} n_{2 p}^{0} \bar{n}_{2 p}^{0} \delta\left(\epsilon_{2 p}-\omega\right) \frac{\chi_{1}^{\prime \prime}(p, \omega)}{-\pi},
\end{aligned}
$$




$$
\begin{aligned}
B_{\mu T}^{11}= & \frac{7 \beta_{1}}{16} \sum_{k, \omega} \frac{2 \pi}{\hbar}|W(k)|^{2} \tilde{\epsilon}_{1 k} 2 D_{1} k^{2} n_{1 k}^{0} \bar{n}_{1 k}^{0} \delta\left(\epsilon_{1 k}-\omega\right) \frac{\chi_{2}^{\prime \prime}(k, \omega)}{-\pi} \\
& +\frac{7}{16} \sum_{p, \omega} \frac{2 \pi}{\hbar}|W(p)|^{2} \delta\left(\epsilon_{2 p}-\omega\right)\left\{\omega\left(n_{B 1}(\omega)-n_{2 p}^{0}\right) \frac{\chi_{1}^{\prime \prime}(p, \omega)}{-\pi}\right. \\
& +2 D_{1} p^{2}\left\{n_{2 p}^{0} \frac{\partial}{\partial \omega}\left[\frac{\chi_{1 \epsilon}^{\prime \prime}(p, \omega)}{-\pi}\right]-\frac{\partial}{\partial \omega}\left[\frac{\chi_{1 \epsilon}^{\prime \prime}(p, \omega)}{-\pi} n_{B 1}(\omega)\right]\right\} \\
& +\left[2 D_{1} p^{2}\left(\epsilon_{01}-\mu_{1}\right)+\omega^{2}\right] \\
& \left.\times\left\{n_{2 p}^{0} \frac{\partial}{\partial \omega}\left[\frac{\chi_{1}^{\prime \prime}(p, \omega)}{-\pi}\right]-\frac{\partial}{\partial \omega}\left[\frac{\chi_{1}^{\prime \prime}(p, \omega)}{-\pi} n_{B 1}(\omega)\right]\right\}\right\}, \\
B_{\mu T}^{12}= & -\frac{7}{16} \sum_{k, \omega} \frac{2 \pi}{\hbar}|W(k)|^{2} \delta\left(\epsilon_{1 k}-\omega\right)\left\{\omega\left(n_{B 2}(\omega)-n_{1 k}^{0}\right) \frac{\chi_{2}^{\prime \prime}(k, \omega)}{-\pi}\right. \\
& +2 D_{2} k^{2}\left\{\left\{n_{1 k}^{0} \frac{\partial}{\partial \omega}\left[\frac{\chi_{2 \epsilon}^{\prime \prime}(k, \omega)}{-\pi}\right]-\frac{\partial}{\partial \omega}\left[\frac{\chi_{2 \epsilon}^{\prime \prime}(k, \omega)}{-\pi} n_{B 2}(\omega)\right]\right\}\right. \\
& +\left[2 D_{2} k^{2}\left(\epsilon_{02}-\mu_{2}\right)+\omega^{2}\right] \\
& \left.\times\left\{n_{1 k}^{0} \frac{\partial}{\partial \omega}\left[\frac{\chi_{2}^{\prime \prime}(k, \omega)}{-\pi}\right]-\frac{\partial}{\partial \omega}\left[\frac{\chi_{2}^{\prime \prime}(k, \omega)}{-\pi} n_{B 2}(\omega)\right]\right\}\right\} \\
& -\frac{7 \beta_{2}}{16} \sum_{p, \omega} \frac{2 \pi}{\hbar}|W(p)|^{2} 2 D_{2} p^{2} \tilde{\epsilon}_{2 p} n_{2 p}^{0} \bar{n}_{2 p}^{0} \delta\left(\epsilon_{2 p}-\omega\right) \frac{\chi_{1}^{\prime \prime}(p, \omega)}{-\pi},
\end{aligned}
$$

where $\chi_{i}^{\prime \prime}(k, \omega)(i=1,2)$ is the imaginary part of the magnon density-density response function

$$
\chi_{i}(k, \omega)=\sum_{\mathbf{p}} \frac{n_{i, \mathbf{p}-}^{0}-n_{i, \mathbf{p}+}^{0}}{\epsilon_{i, \mathbf{p}-}-\epsilon_{i, \mathbf{p}+}+\omega}
$$

and $\chi_{i \epsilon}^{\prime \prime}(k, \omega)$ is the imaginary part of the magnon density-energy-density response function

$$
\chi_{i \epsilon}(k, \omega)=\sum_{\mathbf{p}} \frac{n_{i, \mathbf{p}-}^{0}-n_{i, \mathbf{p}+}^{0}}{\epsilon_{i, \mathbf{p}-}-\epsilon_{i, \mathbf{p}+}+\omega}\left(D_{i} p^{2}+\frac{1}{4} D_{i} k^{2}\right) .
$$

Notice that $B_{\mu \mu}^{12}=-B_{\mu \mu}^{11}$ and $B_{\mu T}^{12}=-B_{\mu T}^{11}$ if the two layers are identical. 


\subsection{Rate of Change of Thermal Current}

Similar to Sec. 5.4, we calculate $\sum_{\mathbf{k}} \mathbf{k} \tilde{\epsilon}_{1 k} I_{A}(\mathbf{k})$ and $\sum_{\mathbf{k}} \mathbf{k} \tilde{\epsilon}_{1 k} I_{B}(\mathbf{k})$. The coefficients before $\mathbf{P}_{i}$ and $\beta \mathbf{P}_{T i}$ yield the matrix elements $B_{T \alpha}^{i j}$ with $\alpha=\mu, T$ and $i, j=1,2$.

$$
\begin{aligned}
& B_{T \mu}^{11}=\frac{7 \beta_{1}}{16} \sum_{k, \omega} \frac{2 \pi}{\hbar}|W(k)|^{2} \tilde{\epsilon}_{1 k} 2 D_{1} k^{2} n_{1 k}^{0} \bar{n}_{1 k}^{0} \delta\left(\epsilon_{1 k}-\omega\right) \frac{\chi_{2}^{\prime \prime}(k, \omega)}{-\pi} \\
& +\frac{7}{16} \sum_{p, \omega} \frac{2 \pi}{\hbar}|W(p)|^{2} \delta\left(\epsilon_{2 p}-\omega\right)\left\{\omega\left(n_{B 1}(\omega)-n_{2 p}^{0}\right) \frac{\chi_{1}^{\prime \prime}(p, \omega)}{-\pi}\right. \\
& +2 D_{1} p^{2}\left\{n_{2 p}^{0} \frac{\partial}{\partial \omega}\left[\frac{\chi_{1 \epsilon}^{\prime \prime}(p, \omega)}{-\pi}\right]-\frac{\partial}{\partial \omega}\left[\frac{\chi_{1 \epsilon}^{\prime \prime}(p, \omega)}{-\pi} n_{B 1}(\omega)\right]\right\} \\
& +\left[2 D_{1} p^{2}\left(\epsilon_{01}-\mu_{1}\right)+\omega^{2}\right] \\
& \left.\times\left\{n_{2 p}^{0} \frac{\partial}{\partial \omega}\left[\frac{\chi_{1}^{\prime \prime}(p, \omega)}{-\pi}\right]-\frac{\partial}{\partial \omega}\left[\frac{\chi_{1}^{\prime \prime}(p, \omega)}{-\pi} n_{B 1}(\omega)\right]\right\}\right\}, \\
& B_{T \mu}^{12}=-\frac{7}{16} \sum_{k, \omega} \frac{2 \pi}{\hbar}|W(k)|^{2} 2 D_{2} k^{2} \tilde{\epsilon}_{1 k} \delta\left(\epsilon_{1 k}-\omega\right) \\
& \times\left\{n_{1 k}^{0} \frac{\partial}{\partial \omega}\left[\frac{\chi_{2}^{\prime \prime}(k, \omega)}{-\pi}\right]-\frac{\partial}{\partial \omega}\left[\frac{\chi_{2}^{\prime \prime}(k, \omega)}{-\pi} n_{B 2}(\omega)\right]\right\} \\
& -\frac{7 \beta_{2}}{16} \sum_{p, \omega} \frac{2 \pi}{\hbar}|W(p)|^{2} n_{2 p}^{0} \bar{n}_{2 p}^{0} \delta\left(\epsilon_{2 p}-\omega\right) \\
& \times\left\{2 D_{2} p^{2} \frac{\chi_{1 \epsilon}^{\prime \prime}(p, \omega)}{-\pi}+\left[2 D_{2} p^{2}\left(\epsilon_{01}-\mu_{1}\right)+\frac{D_{2}}{D_{1}} \omega^{2}\right] \frac{\chi_{1}^{\prime \prime}(p, \omega)}{-\pi}\right\}, \\
& B_{T T}^{11}=\frac{7 \beta_{1}}{16} \sum_{k, \omega} \frac{2 \pi}{\hbar}|W(k)|^{2} 2 D_{1} k^{2} \tilde{\epsilon}_{1 k}^{2} n_{1 k}^{0} \bar{n}_{1 k}^{0} \delta\left(\epsilon_{1 k}-\omega\right) \frac{\chi_{2}^{\prime \prime}(k, \omega)}{-\pi} \\
& +\frac{7}{16} \sum_{p, \omega} \frac{2 \pi}{\hbar}|W(p)|^{2} \delta\left(\epsilon_{2 p}-\omega\right) \\
& \times\left\{\left(n_{B 1}(\omega)-n_{2 p}^{0}\right)\left[6 \omega \frac{\chi_{1 \epsilon}^{\prime \prime}(p, \omega)}{-\pi}+4 \omega\left(\epsilon_{01}-\mu_{1}\right) \frac{\chi_{1}^{\prime \prime}(p, \omega)}{-\pi}\right]\right. \\
& +2 D_{1} p^{2}\left\{n_{2 p}^{0} \frac{\partial}{\partial \omega}\left[\frac{\chi_{1 \epsilon \epsilon}^{\prime \prime}(p, \omega)}{-\pi}\right]-\frac{\partial}{\partial \omega}\left[\frac{\chi_{1 \epsilon \epsilon}^{\prime \prime}(p, \omega)}{-\pi} n_{B 1}(\omega)\right]\right\}
\end{aligned}
$$




$$
\begin{aligned}
& +\left[6 \omega^{2}+4 D_{1} p^{2}\left(\epsilon_{01}-\mu_{1}\right)\right]\left\{n_{2 p}^{0} \frac{\partial}{\partial \omega}\left[\frac{\chi_{1 \epsilon}^{\prime \prime}(p, \omega)}{-\pi}\right]-\frac{\partial}{\partial \omega}\left[\frac{\chi_{1 \epsilon}^{\prime \prime}(p, \omega)}{-\pi} n_{B 1}(\omega)\right]\right\} \\
& +\left[2 \omega^{2}\left(\epsilon_{01}-\mu_{1}\right)+2 D_{1} p^{2}\left(\epsilon_{01}-\mu_{1}\right)^{2}-\omega^{2} D_{1} p^{2}\right] \\
& \left.\times\left\{n_{2 p}^{0} \frac{\partial}{\partial \omega}\left[\frac{\chi_{1}^{\prime \prime}(p, \omega)}{-\pi}\right]-\frac{\partial}{\partial \omega}\left[\frac{\chi_{1}^{\prime \prime}(p, \omega)}{-\pi} n_{B 1}(\omega)\right]\right\}\right\} \\
B_{T T}^{12}= & -\frac{7}{16} \sum_{k, \omega} \frac{2 \pi}{\hbar}|W(k)|^{2} \tilde{\epsilon}_{1 k} \delta\left(\epsilon_{1 k}-\omega\right)\left\{\omega\left(n_{B 2}(\omega)-n_{1 k}^{0}\right) \frac{\chi_{2}^{\prime \prime}(k, \omega)}{-\pi}\right. \\
& +2 D_{2} k^{2}\left\{\left\{n_{1 k}^{0} \frac{\partial}{\partial \omega}\left[\frac{\chi_{2 \epsilon}^{\prime \prime}(k, \omega)}{-\pi}\right]-\frac{\partial}{\partial \omega}\left[\frac{\chi_{2 \epsilon}^{\prime \prime}(k, \omega)}{-\pi} n_{B 2}(\omega)\right]\right\}\right. \\
& \left.+\left[2 D_{2} k^{2}\left(\epsilon_{02}-\mu_{2}\right)+\omega^{2}\right]\left\{n_{1 k}^{0} \frac{\partial}{\partial \omega}\left[\frac{\chi_{2}^{\prime \prime}(k, \omega)}{-\pi}\right]-\frac{\partial}{\partial \omega}\left[\frac{\chi_{2}^{\prime \prime}(k, \omega)}{-\pi} n_{B 2}(\omega)\right]\right\}\right\} \\
& -\frac{7 \beta_{2}}{16} \sum_{p, \omega} \frac{2 \pi}{\hbar}|W(p)|^{2} \tilde{\epsilon}_{2 p} n_{2 p}^{0} \bar{n}_{2 p}^{0} \delta\left(\epsilon_{2 p}-\omega\right) \\
& \times\left\{2 D_{2} p^{2} \frac{\chi_{1 \epsilon}^{\prime \prime}(p, \omega)}{-\pi}+\left[\frac{D_{2}}{D_{1}} \omega^{2}+2 D_{2} p^{2}\left(\epsilon_{01}-\mu_{1}\right)\right] \frac{\chi_{1}^{\prime \prime}(p, \omega)}{-\pi}\right\} .
\end{aligned}
$$

The Onsager relations have been checked: within each layer, the matrix elements of

B satisfy $B_{T \mu}^{11}=B_{\mu T}^{11}$ and $B_{T \mu}^{22}=B_{\mu T}^{22}$; between the two layers that are identical and at thermal equilibrium state (i.e. $\mu=0$ ), we have $B_{T \mu}^{12}=B_{\mu T}^{12}$.

\subsection{Temperature-Dependence of The Drag Resis- tivities}

There are two approaches to studying thermodynamic quantities of quasi-particles: at fixed chemical potential or at fixed number of quasi-particles. In this section, I will discuss temperature-dependence of the drag resistivities in these two cases. In the first case, the chemical potential $\mu_{2}$ in the active layer does not change with temperature. This can be established by applying a continuous microwave field. In the second case, the number of pumped magnons is fixed, therefore, $\mu_{2}$ will be a function of $T$. 
This can be done by applying a microwave pulse. The number of magnons $(N)$ can be regarded fixed in the time window between $100 \mathrm{~ns}$ (thermalization time, used to build quasi-thermal equilibrium state, i.e. to achieve a certain steady distribution characterized by $\mu_{2}$ ) and $1 \mu \mathrm{s}$ (spin-lattice relaxation time, magnons start to decay to phonons) after the pulse is off. $N$ is determined by the power and the frequency of microwave fields. In both cases, the active layer is placed in a thermal bath of temperature $\mathrm{T}$, which acts as a knob for tuning the drag resistivities.

\subsubsection{For fixed chemical potential}

Let layer 1 be the passive layer and layer 2 be the active layer and suppose a steady magnon current is flowing through layer 2 along the negative direction of $\mathbf{M}_{s}(\theta=$ 0). The drag resistivities have been defined in Eq. (5.32). We are interested in the temperature that satisfies $\left(\epsilon_{0 i}-\mu_{i}\right) / k_{B} \ll T \ll T_{c}$, where $T_{c}$ is the Curie temperature and is $550 \mathrm{~K}$ for YIG. This temperature range allows us to approximate the cut-off frequency to be infinity in dealing with thermal integrals, while ensures the temperature be not too low (below magnon energy gap) to thermally excite magnons. We obtain

$$
\left(A^{(i)}\right)^{-1}=\frac{6 \beta_{i}}{\pi^{3}-216 D n_{i}(T) \beta_{i} \zeta[3]}\left(\begin{array}{cc}
18 \zeta[3] & -\pi^{2} \beta_{i} \\
-\pi^{2} \beta_{i} & 12 D n_{i} \pi \beta_{i}^{3}
\end{array}\right)
$$

where $n_{i}(T)$ is the two-dimensional magnon density of layer i at temperature $T$, and the denominator 


$$
\pi^{3}-216 D n_{i}(T) \beta_{i} \zeta[3] \simeq \pi^{3}+\frac{54}{\pi} \ln \left(\frac{\epsilon_{0 i}-\mu_{i}}{k_{B} T}\right)
$$

is negative definitely in the limit of $\epsilon_{0 i}-\mu_{i} \ll k_{B} T$. Unlike in 2 DEGs where $\operatorname{det}\left[A^{(i)}\right]=$ $m \pi-3 n \beta^{2} \epsilon_{F} \hbar^{2} \simeq-3 n \beta^{2} \epsilon_{F} \hbar^{2}$ for temperature much lower than Fermi energy, here we cannot drop $\pi^{3}$ since it is comparable although smaller than $\frac{54}{\pi} \ln \left(\frac{\epsilon_{0 i}-\mu_{i}}{k_{B} T}\right)$ even at room temperature. Define a new matrix $\tilde{\mathbf{B}}^{12}$ that satisfies

$$
\left(\begin{array}{cc}
B_{\mu \mu}^{12} & B_{\mu T}^{12} \\
B_{T \mu}^{12} & B_{T T}^{12}
\end{array}\right)=\frac{7 \beta}{8 \hbar^{2}}\left[\frac{\sqrt{2}}{32} \mu_{0}\left(\frac{g \mu_{B}}{L}\right)^{\frac{3}{2}} \sqrt{\frac{M_{S} D}{\epsilon_{02}-\mu_{2}}}\right]^{2}\left(\begin{array}{cc}
\tilde{B}_{\mu \mu}^{12} & \frac{\tilde{B}_{\mu T}^{12}}{\beta} \\
\frac{\tilde{B}_{T \mu}^{12}}{\beta} & \frac{\tilde{B}_{T T}^{12}}{\beta^{2}}
\end{array}\right)
$$

Such a definition has the advantage that the matrix elements of $\tilde{\mathbf{B}}$ have the same units, $\mathrm{m}^{-4}$, in contrast to the matrix elements of $\mathbf{B}$ which have different units. Suppose the two layers are at the same temperature. $\tilde{\mathbf{B}}^{12}$ is simplified to be

$$
\begin{aligned}
\tilde{B}_{\mu \mu}^{12}= & \frac{\left(\epsilon_{02}-\mu_{2}\right)^{2}}{2 \pi D^{2}} \int d \bar{k} \bar{k}^{3}|\bar{W}(\bar{k}, \bar{d})|^{2} \bar{\chi}_{2}^{\prime \prime}\left(\bar{k}, \bar{k}^{2}+\frac{\epsilon_{01}}{\epsilon_{02}-\mu_{2}}, T\right) n_{1 k}^{0} \bar{n}_{1 k}^{0} \\
& +\frac{\epsilon_{01}^{2}}{2 \pi D^{2}} \int d \bar{p} \bar{p}^{3}|\bar{W}(\bar{p}, \bar{d})|^{2} \bar{\chi}_{1}^{\prime \prime}\left(\bar{p}, \bar{p}^{2}+\frac{\epsilon_{02}}{\epsilon_{01}}, T\right) n_{2 p}^{0} \bar{n}_{2 p}^{0} \\
\tilde{B}_{\mu T}^{12}= & \beta\left\{\frac { ( \epsilon _ { 0 2 } - \mu _ { 2 } ) ^ { 3 } } { 4 \pi D ^ { 2 } } \int d \overline { k } \overline { k } | \overline { W } ( \overline { k } , \overline { d } ) | ^ { 2 } \left\{2 \bar{k}^{2} \bar{\chi}_{2 \epsilon}^{\prime \prime}\left(\bar{k}, \bar{k}^{2}+\frac{\epsilon_{01}}{\epsilon_{02}-\mu_{2}}, T\right)\right.\right. \\
& \left.+\left[2 \bar{k}^{2}+\left(\bar{k}^{2}+\frac{\epsilon_{01}}{\epsilon_{02}-\mu_{2}}\right)^{2}\right] \bar{\chi}_{2}^{\prime \prime}\left(\bar{k}, \bar{k}^{2}+\frac{\epsilon_{01}}{\epsilon_{02}-\mu_{2}}, T\right)\right\} n_{1 k}^{0} \bar{n}_{1 k}^{0} \\
& \left.+\frac{\epsilon_{01}^{3}}{2 \pi D^{2}} \int d \bar{p} \bar{p}^{3}|\bar{W}(\bar{p}, \bar{d})|^{2}\left(\bar{p}^{2}+\frac{\epsilon_{02}-\mu_{2}}{\epsilon_{01}}\right) \bar{\chi}_{1}^{\prime \prime}\left(\bar{p}, \bar{p}^{2}+\frac{\epsilon_{02}}{\epsilon_{01}}, T\right) n_{2 p}^{0} \bar{n}_{2 p}^{0}\right\}
\end{aligned}
$$




$$
\begin{aligned}
\tilde{B}_{T \mu}^{12}= & \beta\left\{\frac{\left(\epsilon_{02}-\mu_{2}\right)^{3}}{2 \pi D^{2}} \int d \bar{k} \bar{k}^{3}|\bar{W}(\bar{k}, \bar{d})|^{2}\left(\bar{k}^{2}+\frac{\epsilon_{01}}{\epsilon_{02}-\mu_{2}}\right)\right. \\
& \times \bar{\chi}_{2}^{\prime \prime}\left(\bar{k}, \bar{k}^{2}+\frac{\epsilon_{01}}{\epsilon_{02}-\mu_{2}}, T\right) n_{1 k}^{0} \bar{n}_{1 k}^{0} \\
& +\frac{\epsilon_{01}^{3}}{4 \pi D^{2}} \int d \bar{p} \bar{p}|\bar{W}(\bar{p}, \bar{d})|^{2}\left\{2 \bar{p}^{2} \bar{\chi}_{1 \epsilon}^{\prime \prime}\left(\bar{p}, \bar{p}^{2}+\frac{\epsilon_{02}}{\epsilon_{01}}, T\right)\right. \\
& \left.\left.+\left[2 \bar{p}^{2}+\left(\bar{p}^{2}+\frac{\epsilon_{02}}{\epsilon_{01}}\right)^{2}\right] \bar{\chi}_{1}^{\prime \prime}\left(\bar{p}, \bar{p}^{2}+\frac{\epsilon_{02}}{\epsilon_{01}}, T\right)\right\} n_{2 p}^{0} \bar{n}_{2 p}^{0}\right\}, \\
\tilde{B}_{T T}^{12}= & \beta^{2}\left\{\frac{\left(\epsilon_{02}-\mu_{2}\right)^{4}}{4 \pi D^{2}} \int d \bar{k} \bar{k}|\bar{W}(\bar{k}, \bar{d})|^{2}\left(\bar{k}^{2}+\frac{\epsilon_{01}}{\epsilon_{02}-\mu_{2}}\right)\right. \\
& \times\left\{2 \bar{k}^{2} \bar{\chi}_{2 \epsilon}^{\prime \prime}\left(\bar{k}, \bar{k}^{2}+\frac{\epsilon_{01}}{\epsilon_{02}-\mu_{2}}, T\right)\right. \\
& \left.+\left[2 \bar{k}^{2}+\left(\bar{k}^{2}+\frac{\epsilon_{01}}{\epsilon_{02}-\mu_{2}}\right)^{2}\right] \bar{\chi}_{2}^{\prime \prime}\left(\bar{k}, \bar{k}^{2}+\frac{\epsilon_{01}}{\epsilon_{02}-\mu_{2}}, T\right)\right\} n_{1 k}^{0} \bar{n}_{1 k}^{0} \\
& +\frac{\epsilon_{01}^{4}}{4 \pi D^{2}} \int d \bar{p} \bar{p}|\bar{W}(\bar{p}, \bar{d})|^{2}\left(\bar{p}^{2}+\frac{\epsilon_{02}}{\epsilon_{01}}\right)\left\{2 \bar{p}^{2} \bar{\chi}_{1 \epsilon}^{\prime \prime}\left(\bar{p}, \bar{p}^{2}+\frac{\epsilon_{02}}{\epsilon_{01}}, T\right)\right. \\
& \left.\left.+\left[2 \bar{p}^{2}+\left(\bar{p}^{2}+\frac{\epsilon_{02}}{\epsilon_{01}}\right)^{2}\right] \bar{\chi}_{1}^{\prime \prime}\left(\bar{p}, \bar{p}^{2}+\frac{\epsilon_{02}}{\epsilon_{01}}, T\right)\right\} n_{2 p}^{0} \bar{n}_{2 p}^{0}\right\},
\end{aligned}
$$

where $n_{i k}=\frac{1}{\operatorname{Exp}\left[\frac{D k^{2}+\epsilon_{0 i}-\mu_{i}}{k_{B} T}\right]-1}$ is the distribution function of magnons in layer $\mathrm{i}$, and we have used $\mu_{1}=0$ since layer 1 is at thermal equilibrium. The quantities with bar are dimensionless (for example, $\bar{k}=k / \sqrt{\frac{\epsilon_{02}-\mu_{2}}{D}}$ and $\bar{p}=p / \sqrt{\frac{\epsilon_{01}}{D}}$ ), except for $\bar{n}_{i k}=1+n_{i k}$ . $\bar{W}(\bar{k}, \bar{d})$ is the dimensionless amplitude of three-magnon interaction.

$$
\bar{W}(\bar{k}, \bar{d})=\frac{1}{\bar{k}} e^{-\bar{k}(\bar{d}-\bar{L})}\left(1-e^{-\bar{k} \bar{L}}\right)^{2}
$$

where $\bar{d}=d \sqrt{\frac{\epsilon_{02}-\mu_{2}}{D}}$ is the dimensionless distance between the films, and $\bar{L}=$ $L \sqrt{\frac{\epsilon_{02}-\mu_{2}}{D}}$ is the dimensionless thickness of each film.

$\bar{\chi}^{\prime \prime}$ and $\bar{\chi}_{\epsilon}^{\prime \prime}$ denote the imaginary parts of the dimensionless magnon response func- 
tions at finite temperature,

$$
\begin{aligned}
\bar{\chi}^{\prime \prime}(\bar{k}, \bar{\omega}, T)= & 4 \pi D \chi^{\prime \prime}(\bar{k}, \bar{\omega}, T) \\
= & -\frac{1}{\bar{k}} \int_{0}^{\infty} \frac{x\left(\epsilon_{0}-\mu\right) d x}{2 k_{B} T \sinh ^{2}\left[\left(x^{2}+1\right) \frac{\epsilon_{0}-\mu}{2 k_{B} T}\right]} \\
& \times\left[\Theta\left(x-\left|\nu_{-}\right|\right) \sqrt{x^{2}-\nu_{-}^{2}}-\Theta\left(x-\left|\nu_{+}\right|\right) \sqrt{x^{2}-\nu_{+}^{2}}\right], \\
\bar{\chi}_{\epsilon}^{\prime \prime}(\bar{k}, \bar{\omega}, T)= & \frac{4 \pi D}{\epsilon_{0}-\mu} \chi_{\epsilon}^{\prime \prime}(\bar{k}, \bar{\omega}, T) \\
= & -\frac{1}{\bar{k}} \int_{0}^{\infty} \frac{x\left(\epsilon_{0}-\mu\right) d x}{2 k_{B} T \sinh ^{2}\left[\left(x^{2}+1\right) \frac{\epsilon_{0}-\mu}{2 k_{B} T}\right]} \\
& \times\left[\Theta\left(x-\left|\nu_{-}\right|\right) \sqrt{x^{2}-\nu_{-}^{2}}\left(\frac{\bar{k}^{2}}{6}+\frac{x^{2}}{3}+\frac{\bar{\omega}}{6}+\frac{\bar{\omega}^{2}}{6 \bar{q}^{2}}\right)\right. \\
& \left.-\Theta\left(x-\left|\nu_{+}\right|\right) \sqrt{x^{2}-\nu_{+}^{2}}\left(\frac{\bar{k}^{2}}{6}+\frac{x^{2}}{3}-\frac{\bar{\omega}}{6}+\frac{\bar{\omega}^{2}}{6 \bar{q}^{2}}\right)\right],
\end{aligned}
$$

with $\nu_{ \pm}=\frac{\bar{\omega}}{2 \bar{k}} \pm \frac{\bar{k}}{2}$. The $\omega$-dependence of the response functions has been integrated out in Eqs. (5.54) to (5.57) by virtue of the energy conservations $\delta\left(\epsilon_{1 k}-\omega\right)$ in process $W_{1}$ and $\delta\left(\epsilon_{2 k}-\omega\right)$ in process $W_{2}$, as shown in Fig. 5.3. To study the temperature-dependence of $\mathbf{C}^{12}$, I will drop the factors that do not depend on temperature and define a new matrix $\tilde{\mathbf{C}}^{12}$ such that $\mathbf{C}=\frac{2 D}{\hbar}\left(\mathbf{A}^{(1)}\right)^{-1} \mathbf{B}^{12}\left(\mathbf{A}^{(2)}\right)^{-1}=$ $63 \hbar\left[\frac{\sqrt{2}}{8} \mu_{0}\left(\frac{g \mu_{B}}{L}\right)^{\frac{3}{2}} \sqrt{\frac{M s}{\epsilon_{02}-\mu_{2}}}\right]^{2} \tilde{\mathbf{C}}^{12}$. Therefore, 


$$
\begin{aligned}
\tilde{C}_{\mu \mu}^{12}= & \frac{D^{2} \beta^{3}}{\left(\pi^{3}-216 D n_{1}(T) \beta \zeta[3]\right)\left(\pi^{3}-216 D n_{2}(T) \beta \zeta[3]\right)} \\
& \times\left\{\pi^{4} \tilde{B}_{T T}^{12}-18 \zeta[3]\left(\pi^{2} \tilde{B}_{T \mu}^{12}+\pi^{2} \tilde{B}_{\mu T}^{12}-18 \zeta[3] \tilde{B}_{\mu \mu}^{12}\right)\right\}, \\
\tilde{C}_{\mu T}^{12}= & \frac{D^{2} \pi \beta^{4}}{\left(\pi^{3}-216 D n_{1}(T) \beta \zeta[3]\right)\left(\pi^{3}-216 D n_{2}(T) \beta \zeta[3]\right)}\left\{\pi^{3} \tilde{B}_{T \mu}^{12}\right. \\
& \left.-6\left[2 D n_{2}(T) \pi^{2} \beta \tilde{B}_{T T}^{12}+3 \zeta[3]\left(\pi \tilde{B}_{\mu \mu}^{12}-12 D n_{2}(T) \beta \tilde{B}_{\mu T}^{12}\right)\right]\right\}, \\
\tilde{C}_{T \mu}^{12}= & \frac{D^{2} \pi \beta^{4}}{\left(\pi^{3}-216 D n_{1}(T) \beta \zeta[3]\right)\left(\pi^{3}-216 D n_{2}(T) \beta \zeta[3]\right)}\left\{\pi^{3} \tilde{B}_{\mu T}^{12}\right. \\
& \left.-6\left[2 D n_{1}(T) \pi^{2} \beta \tilde{B}_{T T}^{12}+3 \zeta[3]\left(\pi \tilde{B}_{\mu \mu}^{12}-12 D n_{1}(T) \beta \tilde{B}_{T \mu}^{12}\right)\right]\right\}, \\
\tilde{C}_{T T}^{12}= & \frac{D^{2} \pi^{2} \beta^{5}}{\left(\pi^{3}-216 D n_{1}(T) \beta \zeta[3]\right)\left(\pi^{3}-216 D n_{2}(T) \beta \zeta[3]\right)}\left\{\pi^{2} \tilde{B}_{\mu \mu}^{12}\right. \\
& \left.+12 D \beta\left[-n_{1}(T) \pi \tilde{B}_{T \mu}^{12}-n_{2}(T) \pi \tilde{B}_{\mu T}^{12}+12 D n_{1}(T) n_{2}(T) \beta \tilde{B}_{T T}^{12}\right]\right\} .
\end{aligned}
$$

In the high temperature limit $\left(T \gg \epsilon_{0 i}-\mu_{i}\right)$,

$$
\begin{aligned}
n_{i}(T) & =\sum_{\mathbf{k}} n_{i k}=-\frac{k_{B} T}{4 \pi D} \ln \left(1-e^{\frac{\epsilon_{0 i}-\mu_{i}}{k_{B} T}}\right) \approx-\frac{k_{B} T}{4 \pi D} \ln \left(\frac{\epsilon_{0 i}-\mu_{i}}{k_{B} T}\right) \\
n_{1 k} \bar{n}_{1 k} & =\frac{1}{4 \sinh ^{2}\left[\left(\bar{k}^{2}+\frac{\epsilon_{01}}{\epsilon_{02}-\mu_{2}}\right) \frac{\epsilon_{02}-\mu_{2}}{2 k_{B} T}\right]} \approx \frac{1}{4\left(\bar{k}^{2}+\frac{\epsilon_{01}}{\epsilon_{02}-\mu_{2}}\right)^{2}}\left(\frac{2 k_{B} T}{\epsilon_{02}-\mu_{2}}\right)^{2} \\
n_{2 p} \bar{n}_{2 p} & =\frac{1}{\left.4 \sinh ^{2}\left(\bar{p}^{2}+\frac{\epsilon_{02}-\mu_{2}}{\epsilon_{01}}\right) \frac{\epsilon_{01}}{2 k_{B} T}\right]} \approx \frac{1}{4\left(\bar{p}^{2}+\frac{\epsilon_{02}-\mu_{2}}{\epsilon_{01}}\right)^{2}}\left(\frac{2 k_{B} T}{\epsilon_{01}}\right)^{2}
\end{aligned}
$$

Substituting these approximations into Eqs. (5.54) to (5.57), we get the temperature dependence of matrix elements $\tilde{B}_{\alpha \beta}^{12}(\alpha, \beta=\mu, T)$, that is

$$
\tilde{B}_{\mu \mu}^{12} \propto T^{3}, \quad \tilde{B}_{\mu T}^{12} \propto T^{2}, \quad \tilde{B}_{T \mu}^{12} \propto T^{2}, \quad \tilde{B}_{T T}^{12} \propto T
$$

In the high temperature limit, terms with higher order of $T$ are more important. 
Therefore, we keep only $\tilde{B}_{\mu \mu}^{12}$ and simplify the drag resistivities as

$$
\begin{aligned}
\tilde{C}_{\mu \mu}^{12} & =\frac{D^{2} \beta^{3} \pi^{2}(18 \zeta[3])^{2} \tilde{B}_{\mu \mu}^{12}}{\left\{\pi^{2}+54 \ln \left(\beta \epsilon_{01}\right) \zeta[3]\right\}\left\{\pi^{2}+54 \ln \left[\beta\left(\epsilon_{02}-\mu_{2}\right)\right] \zeta[3]\right\}}, \\
\tilde{C}_{\mu T}^{12} & =\tilde{C}_{T \mu}^{12}=\frac{-D^{2} \beta^{4} \pi^{2} 18 \zeta[3] \tilde{B}_{\mu \mu}^{12}}{\left\{\pi^{2}+54 \ln \left(\beta \epsilon_{01}\right) \zeta[3]\right\}\left\{\pi^{2}+54 \ln \left[\beta\left(\epsilon_{02}-\mu_{2}\right)\right] \zeta[3]\right\}}, \\
\tilde{C}_{T T}^{12} & =\frac{D^{2} \beta^{5} \pi^{4} \tilde{B}_{\mu \mu}^{12}}{\left\{\pi^{2}+54 \ln \left(\beta \epsilon_{01}\right) \zeta[3]\right\}\left\{\pi^{2}+54 \ln \left[\beta\left(\epsilon_{02}-\mu_{2}\right)\right] \zeta[3]\right\}}
\end{aligned}
$$

The matrix elements of $\tilde{\mathbf{C}}^{12}$ as a function of $T$ have been shown in Fig. 5.7. It shows that the high temperature approximation works pretty well for $T>10 \mathrm{~K}$ (i.e. $\frac{\epsilon_{0 i}-\mu_{i}}{k_{B} T}<0.1$ with the parameters given in the caption). For $T<100 \mathrm{~K}$, there is a sharp increase of the drag resistivities since the Bose-Einstein distribution yields $n_{i}(T) \propto T \ln T$. The numerical results also show a critical temperature, below which the drag resistivities drop again. This is because no magnon can be thermally excited when the temperature is smaller than the energy gap $\left(\epsilon_{0 i}\right)$. In the extreme case, when $T=0 \mathrm{~K}$, there is no magnon at all and the drag resistivity becomes zero. It is also worth noting that the temperature dependence will be quite different for bosons with conserved particle number. In the magnon case, the chemical potential is controlled by the pumping power and pumping rate of the external source, which can be independent on temperature. However, for bosons whose particle number is fixed, the chemical potential will depend on temperature. For example, Duine and Stoof have found the drag resistivity between two spin-species in cold atoms has a $T^{-2}$ dependence [33]. 

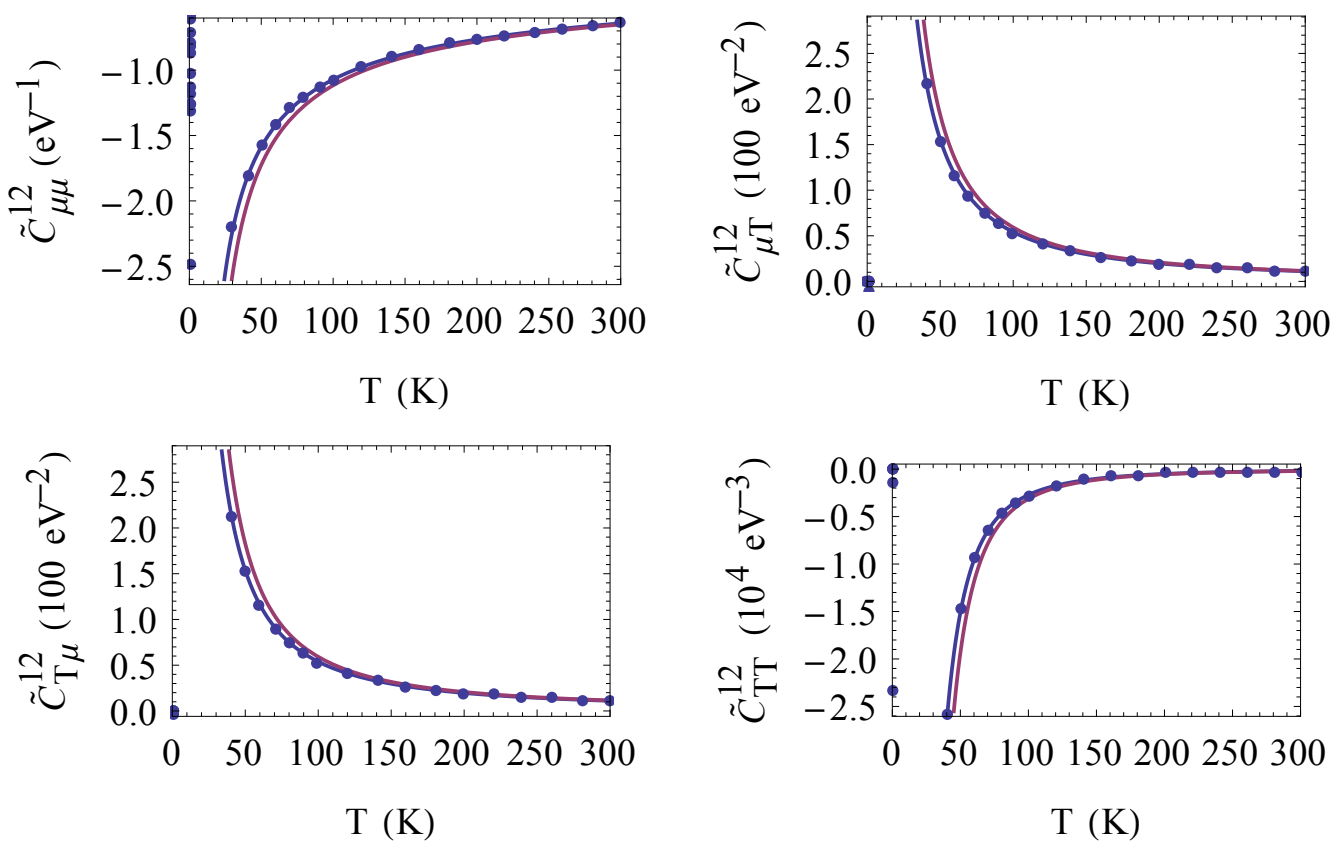

Figure 5.7: The matrix elements of $\tilde{\mathbf{C}}^{12}$ as a function of $T$. The dots are the exact numerical results and the solid lines are the analytical results in the high temperature approximation. Blue line: use the full expression Eq, (5.62) to (5.65). Purple line: keep only $\tilde{B}_{\mu \mu}^{12}$ as in Eq. (5.70) to (5.72). The parameters has been used to generate the function are $\epsilon_{01}=0.5 \mathrm{~K}, \epsilon_{02}=1 \mathrm{~K}, \mu_{1}=0 \mathrm{~K}, \mu_{2}=0.2 \mathrm{~K}, d=6 \mathrm{~nm}, L=3 \mathrm{~nm}$, $k_{B}=8.61 \times 10^{-5} \mathrm{eV} \cdot \mathrm{K}^{-1}$, and $D=3.01 \times 10^{-17} \mathrm{~K} \cdot \mathrm{m}^{2}$. 


\subsubsection{For fixed number of pumped magnons}

For two-dimensional magnon gases, the number of magnons per unit area can be obtained analytically,

$$
n=\sum_{k} \frac{1}{e^{\beta\left(D k^{2}+\epsilon_{0}-\mu\right)}-1}=\frac{-k_{B} T}{4 \pi D} \ln \left(1-e^{\mu-\epsilon_{0}}\right) .
$$

At thermal equilibrium, the chemical potential $\mu=0$. Let $\delta n$ be the number of pumped magnons per unit area, we have $\delta n=n(\mu, T)-n(0, T)$ and the chemical potential becomes a function of $\delta n$ and $T$,

$$
\mu(\delta n, T)=\ln \left[1-\left(1-e^{-\epsilon_{0}}\right) e^{\frac{-4 \pi \delta n}{k_{B} T}}\right]+\epsilon_{0} .
$$

This formula shows that $\mu$ increases with increasing $\delta n$ or decreasing temperature (two ways towards Bose-Einstein condensation) and it is always less than $\epsilon_{0}$.

In Fig. 5.8 we compare the drag resistivities for fixed chemical potential $\mu_{2}$ (blue points) and for fixed number of pumped magnons (purple points). The results show that the most significant difference is in $\tilde{C}_{\mu \mu}^{12}$ in the low temperature range. If the number of the pumped magnons is fixed, lowering the temperature will lead to the formation of Bose-Einstein condensation (BEC) below $9 \mathrm{~K}$ (for the parameters we are using), and therefore, the drag resistivity cannot gain as much enhancement as in the case of fixed chemical potential before it starts to fall again. What happens when $\mathrm{BEC}$ is formed is beyond the reach of our model, since the magnons in the BEC state cannot be described by the Bose-Einstein distribution. 

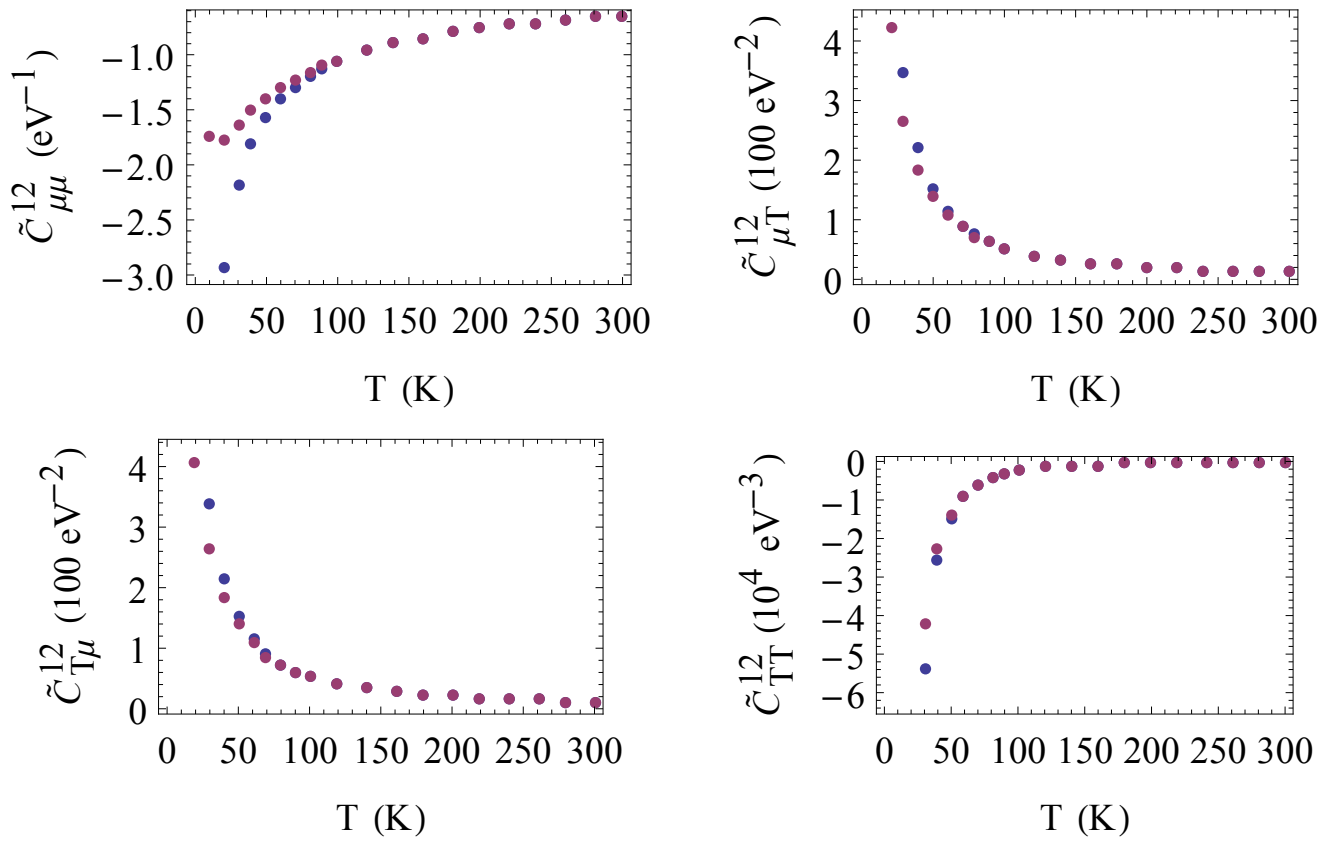

Figure 5.8: Comparison of the drag resistivities as a function of $T$ for fixed $\mu_{2}$ (blue dots) and those for fixed number of pumped magnons (purple dots). 


\subsection{Distance Dependence of the drag resistivities}

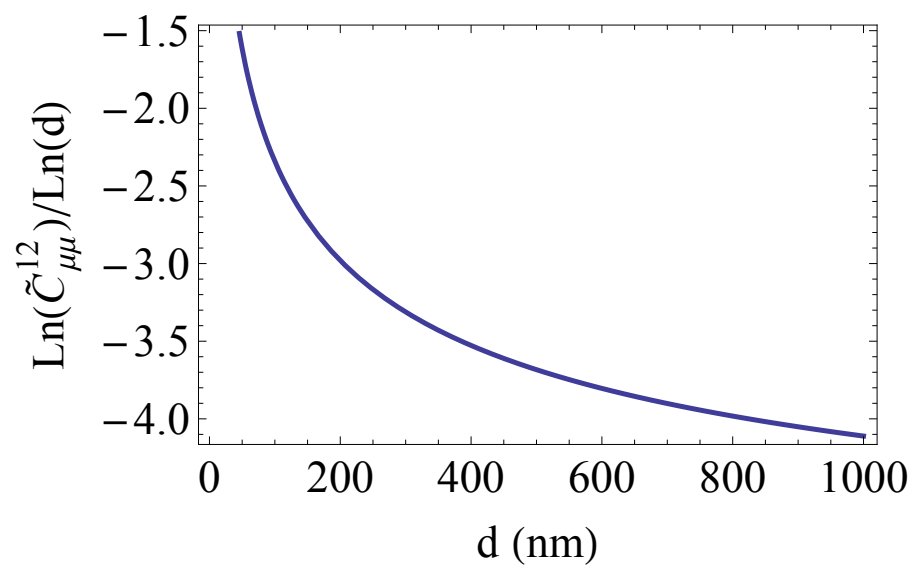

Figure 5.9: The power law of the drag resistivity with respect to $d$.

As discussed in Sec. 5.6, we are allowed to use the high temperature approximation at room temperature. According to Eq. (5.70) to (5.72), the four resistivities share the same $d$-dependence, because only $\tilde{B}_{\mu \mu}^{12}$ is a function of $d$. Therefore, take $\tilde{C}_{\mu \mu}^{12}$ as an example, we study its power law in $d$ in Fig. 5.9. Unlike the Coulomb drag whose $\rho_{D} \propto d^{-4}$, the resistivity for magnon drag decreases more slowly when increasing the distance between the two layers.

\subsection{Estimation for Measurable Quantities}

Since there is no big difference between the drag resistivities for fixed number of pumped magnons and that for fixed chemical potential. We will use the formula obtained for a fixed chemical potential in layer 2 to estimate the measurable quantities $\left(\nabla M_{z 1}\right.$ and $\left.\nabla T_{1}\right)$ in layer 1. 
To estimate how large of $\nabla \mu_{1}$ can be induced by $\mathbf{j}_{2}$, one needs to use the relation that

$$
\nabla \mu_{1}=-C_{\mu \mu}^{12} \mathbf{j}_{2}-C_{\mu T}^{12} \mathbf{j}_{Q 2}
$$

Suppose layer 2 is maintained at a uniform temperature, then $\mathbf{j}_{Q 2}$ is related to $\mathbf{j}_{2}$ by

$$
\frac{\mathbf{j}_{Q 2}}{\mathbf{j}_{2}}=\frac{A_{T \mu}}{A_{\mu \mu}}=\frac{\pi}{12 n_{2} D \beta^{2}} .
$$

At room temperature $\left(n_{2}=4.7 \times 10^{18} \mathrm{~m}^{-2}\right), \mathbf{j}_{Q 2} / \mathbf{j}_{2} \sim 0.01 \mathrm{eV}$, and $C_{\mu T}^{12} / C_{\mu \mu}^{12} \sim-17$ $\mathrm{eV}^{-1}$. Therefore, one can neglect the contribution of thermal current in estimating the effect of magnon drag, so that $\nabla \mu_{1}=-C_{\mu \mu}^{12} \mathbf{j}_{2}$, with

$$
C_{\mu \mu}^{12}=\frac{63 \hbar}{D}\left[\frac{\sqrt{2}}{32} \mu_{0}\left(\frac{g \mu_{B}}{L}\right)^{\frac{3}{2}} \sqrt{\frac{M_{S} D}{\epsilon_{02}-\mu_{2}}}\right]^{2} \tilde{C}_{\mu \mu}^{12}=-3.7 \times 10^{-42}(\mathrm{~J} \cdot \mathrm{s})
$$

The measurable quantity in the passive layer is $M_{z}=M_{s}-\frac{g \mu_{B}}{L} \sum_{\mathbf{k}} n_{k}(\mathbf{r}) . \quad(L$ in the denominator is because $M_{s}$ is magnetic moment per volume and the summation over $\mathbf{k}$ is in two-dimension.) Therefore, $\nabla M_{z}$ is related to $\nabla \mu$ by

$$
\begin{aligned}
\nabla M_{z} & =-\frac{g \mu_{B}}{L} \sum_{\mathbf{k}} \nabla \frac{1}{e^{\frac{\epsilon_{k}-\mu(\mathbf{r})}{k_{B} T}}-1} \\
& =-\frac{g \mu_{B}}{L} \sum_{\mathbf{k}}\left(-\frac{\partial n_{k}}{\partial \epsilon_{k}}\right) \nabla \mu .
\end{aligned}
$$


Let $\mathbf{j}_{s}=-g \mu_{B} \mathbf{j}$, we obtain

$$
\begin{aligned}
\frac{\boldsymbol{\nabla} M_{z 1}}{\mathbf{j}_{s 2}} & =\frac{1}{L} \sum_{\mathbf{k}}\left(-\frac{\partial n_{1 k}}{\partial \epsilon_{1 k}}\right) C_{\mu \mu}^{12} \\
& =\frac{1}{2 \pi L} \frac{\epsilon_{01}}{D} \int d \bar{k} \frac{\bar{k} e^{\epsilon_{01}\left(\bar{k}^{2}+1\right) / T}}{k_{B} T\left(e^{\epsilon_{01}\left(\bar{k}^{2}+1\right) / T}-1\right)^{2}} C_{\mu \mu}^{12} \simeq-8.3 \times 10^{7} s \cdot m^{-3}(
\end{aligned}
$$

The value is estimated at room temperature. Suppose the magnon spin current in layer 2 is carried by parametrically pumped magnons with density of $10^{18} \mathrm{~cm}^{-3}$ and average velocity $100 \mathrm{~m} / \mathrm{s}$, then $j_{s 2}=\left[g \mu_{B}\right] n L v \sim g \mu_{B} 3 \times 10^{17} \mathrm{~m}^{-1} \mathrm{~s}^{-1}$. Substituting it into Eq. (5.79), we obtain

$$
\delta M_{z 1}:=\nabla M_{z 1} \cdot 1 \mathrm{~mm}=g \mu_{B} 2 \times 10^{21} \mathrm{~m}^{-3}=0.3 \mathrm{~A} / \mathrm{m} .
$$

The change of magnetization in the passive layer due to magnon drag in the active layer is about $10^{-6}$ of the saturation magnetization of YIG $\left(M_{s}=1.4 \times 10^{5} \mathrm{~A} / \mathrm{m}\right)$. The effect can be enhanced by enlarging the sample and lowering the temperature.

Now let us turn to see the induced temperature gradient in the passive layer,

$$
\frac{\nabla T_{1}}{T_{1}}=-C_{\mu T}^{12} \mathbf{j}_{2}-C_{T T}^{12} \mathbf{j}_{Q 2} .
$$

Noting that $\mathbf{j}_{Q 2} / \mathbf{j}_{2} \sim 10^{-7} \mathrm{eV}$ and $C_{T T}^{12} / C_{T \mu}^{12} \sim-17 \mathrm{eV}$ at room temperature, we again neglect the thermal current term and get

$$
\nabla T_{1} \simeq-C_{\mu T}^{12} j_{2} T_{1}=0.036 \mathrm{~K} / \mathrm{m} .
$$




\subsection{Conclusion}

To conclude, we study the drag effect between two layers of two-dimensional magnon gases. It behaves quite different from ordinary Coulomb drag due to the Bose-Einstein statistics and the non-conservation of the magnon number. The drag resistivity can be enhanced by orders of magnitude by decreasing the temperature, whereas the Coulomb drag resistivity decreases as $T^{2}$. If a zero resistivity is observed at a temperature far above magnon energy gap. It may be an indication of Bose-Einstein condensation. To get the maximum drag, one should make the spin-wave spin current in the active layer propagate parallel or anti-parallel to the equilibrium magnetization. The magnon drag resistivities decrease more slowly than the Coulomb drag, which decreases as $1 / d^{4}$, when increasing the distance between the layers.

We also estimate the change of magnetization in the passive layer induced by a magnon current of $g \mu_{B} \times 3 \times 10^{17} \mathrm{~m}^{-1} \mathrm{~s}^{-1}$ propagating in the active layer. At room temperature, we expect to see a change of magnetization of about $0.3 \mathrm{~A} / \mathrm{m}$ along a

distance of $1 \mathrm{~mm}$. The magnitude of the change can be increased by a factor of 10 if doing the experiments at a $T \sim 30 \mathrm{~K}$. 


\section{Appendix A}

\section{Superexchange Model}

\section{A.1 Hamiltonian}

In the simplest model for superexchange interaction, we consider a system with two metal ions connected by an oxygen ligand, which is a four electrons on three sites problem.

Suppose each metal ion carries one localized spin-1/2. Then the Hamiltonian is

$$
\begin{aligned}
H= & -t \sum_{i=1}^{2} \sum_{\sigma}\left(c_{i \sigma}^{\dagger} c_{0 \sigma}+h . c .\right)+\epsilon_{1} \sum_{i=1}^{2} \sum_{\sigma} c_{i \sigma}^{\dagger} c_{i \sigma}+\epsilon_{0} \sum_{\sigma} c_{0 \sigma}^{\dagger} c_{0 \sigma} \\
& +U \sum_{i=1}^{2} \sum_{\sigma} c_{i \sigma}^{\dagger} c_{i \sigma} c_{i \bar{\sigma}}^{\dagger} c_{i \bar{\sigma}}
\end{aligned}
$$

where $c\left(c^{\dagger}\right)$ is the creation (annihilation) operator of ligand electrons, which can hop forth and back between oxygen anions and the metal ions, $\epsilon_{1}$ and $\epsilon_{0}$ are the orbital energies of an metal ion and the oxygen ligand, respectively. We are interested in $3 \mathrm{~d}$ 
metal and the repulsion energy on $3 \mathrm{~d}$-orbital is much larger than that on $2 \mathrm{p}$-orbital, so repulsion energy $U$ accounts only for two electrons appear in the same metal ion and the repulsion energy on the oxygen is neglected.

When we apply a uniform electric field to the system, the hopping part in Eq. (A.1) will be moderated by a phase factor due to spin-orbit coupling, that is

$$
\begin{aligned}
H= & -t \sum_{\sigma}\left(c_{1 \sigma}^{\dagger} c_{0 \sigma} e^{-i \theta \sigma}+c_{2 \sigma}^{\dagger} c_{0 \sigma} e^{i \theta \sigma}+\text { h.c. }\right)+\epsilon_{1} \sum_{i=1}^{2} \sum_{\sigma} c_{i \sigma}^{\dagger} c_{i \sigma}+\epsilon_{0} \sum_{\sigma} c_{0 \sigma}^{\dagger} c_{0 \sigma} \\
& +U \sum_{i=1}^{2} \sum_{\sigma} c_{i \sigma}^{\dagger} c_{i \sigma} c_{i \bar{\sigma}}^{\dagger} c_{i \bar{\sigma}} .
\end{aligned}
$$

Note that the hamiltonian conserve $S_{z}^{\text {total }}$, we can diagonalize it in three subspaces: $S_{z}^{\text {total }}=0$ and $S_{z}^{\text {total }}= \pm 1$. Fig.A.1 and Fig.A.2 show the basis for these three subspaces.

$\left(H_{i j}\right)=\left(\begin{array}{ccccccccc}0 & 0 & 0 & 0 & t e^{-i \theta} & 0 & t e^{-i \theta} & 0 & 0 \\ 0 & 0 & 0 & 0 & 0 & t e^{i \theta} & 0 & t e^{i \theta} & 0 \\ 0 & 0 & U & 0 & -t e^{i \theta} & t e^{-i \theta} & 0 & 0 & 0 \\ 0 & 0 & 0 & U & 0 & 0 & -t e^{i \theta} & t e^{-i \theta} & 0 \\ t e^{i \theta} & 0 & -t e^{-i \theta} & 0 & V & 0 & 0 & 0 & -t e^{-i \theta} \\ 0 & t e^{-i \theta} & t e^{i \theta} & 0 & 0 & V & 0 & 0 & t e^{i \theta} \\ t e^{i \theta} & 0 & 0 & -t e^{-i \theta} & 0 & 0 & V & 0 & -t e^{-i \theta} \\ 0 & t e^{-i \theta} & 0 & t e^{i \theta} & 0 & 0 & 0 & V & t e^{i \theta} \\ 0 & 0 & 0 & 0 & -t e^{i \theta} & t e^{-i \theta} & -t e^{i \theta} & t e^{-i \theta} & 2 V\end{array}\right),\left(S_{z}^{t o t a l}=0\right)$

where $V=\epsilon_{1}-\epsilon_{0}+U$ and the zero energy point has been chosen to be $2 \epsilon_{1}+2 \epsilon_{0}$. In 
large $U$ limit, we can use perturbation theory to get the effective hamiltonian in the subspace where the metal sites are singly occupied. Detailed calculation shows that we need to keep up to the fourth order of $t$. The Van Vleck transformation is used in the following section to avoid the complicated process in dealing with higher order degenerate perturbation.

The matrix elements for $S_{z}^{\text {total }}= \pm 1$ are

$$
\begin{aligned}
\left(H_{i j}\right) & =\left(\begin{array}{ccc}
0 & t e^{-i \theta} & t e^{i \theta} \\
t e^{i \theta} & V & 0 \\
t e^{-i \theta} & 0 & V
\end{array}\right),\left(S_{z}^{\text {total }}=1\right) \\
\left(H_{i j}\right) & =\left(\begin{array}{ccc}
0 & t e^{i \theta} & t e^{-i \theta} \\
t e^{-i \theta} & V & 0 \\
t e^{i \theta} & 0 & V
\end{array}\right),\left(S_{z}^{\text {total }}=-1\right)
\end{aligned}
$$

with eigenvalues: $V,\left(V \pm \sqrt{V^{2}+8 t^{2}}\right) / 2$. However, we will use the perturbation theory up to fourth order again in this subspace in order to keep consistency with the $S_{z}^{\text {total }}=0$ subspace.

\section{A.2 The Van Vleck Transformation}

The Van Vleck transformation [34] is a uniform transformation which decoupling the states of interest, labelled by $m$, from the other states, labelled by $n$. In other words, it changes the original Hamiltonian into block diagonal matrix, which allows us to treat each block separately. 
In general, we introduce a uniform transformation $\mathbf{T}(\lambda)=\mathbf{e}^{\mathbf{i} \lambda \mathbf{S}}$ where $\lambda$ is between 0 and 1 and $\mathbf{S}=\mathbf{S}_{\mathbf{1}}+\lambda \mathbf{S}_{\mathbf{2}}+\lambda^{\mathbf{2}} \mathbf{S}_{\mathbf{3}} \ldots$, a Hermitian matrix which eliminates the matrix element connecting states $m$ and $n$. And we require that $\mathbf{S}_{\mathbf{m m}^{\prime}}=\mathbf{S}_{\mathbf{n n}}=\mathbf{0}$. Then we get the new Hamiltonian

$$
\mathbf{G}(\lambda)=\mathbf{G}^{0}+\lambda \mathbf{G}^{\prime}+\lambda^{2} \mathbf{G}^{\prime \prime}+\ldots=\mathbf{T}^{\dagger} \mathbf{H T},
$$

where $\mathbf{H}=\mathbf{H}^{\mathbf{0}}+\lambda \mathbf{H}^{\prime}+\lambda^{\mathbf{2}} \mathbf{H}^{\prime \prime}+\ldots$

In our model, $\mathbf{H}=\mathbf{H}^{\mathbf{0}}+\mathbf{H}^{\prime}$ and the states of interest have $H_{m m^{\prime}}^{\prime}=H_{n n^{\prime}}^{\prime}=0$ and the same unperturbed energy $E_{m}^{0}$. Equating the coefficient of various powers of $\lambda$, we obtain

$$
\begin{aligned}
G_{m m^{\prime}}= & E_{m}^{0} \delta_{m m^{\prime}}+\lambda^{2} \frac{H_{m n}^{\prime} H_{n m^{\prime}}^{\prime}}{E_{m}-E n}+\lambda^{4} \frac{H_{m n}^{\prime} H_{n n^{\prime}}^{\prime} H_{n^{\prime} n^{\prime \prime}}^{\prime} H_{n^{\prime \prime} m^{\prime}}^{\prime}}{\left(E_{m}-E_{n}\right)\left(E_{m}-E_{n^{\prime}}\right)\left(E_{m}-E_{n^{\prime \prime}}\right)} \\
& -\frac{1}{2} \lambda^{4} H_{m n}^{\prime} H_{n m^{\prime}}^{\prime} H_{m^{\prime} n^{\prime}}^{\prime} H_{n^{\prime} m^{\prime}}^{\prime} \\
& \times\left[\frac{1}{\left(E_{m}-E_{n}\right)\left(E_{m}-E_{n^{\prime}}\right)^{2}}+\frac{1}{\left(E_{m}-E_{n}\right)^{2}\left(E_{m}-E_{n^{\prime}}\right)}\right] .
\end{aligned}
$$

Here we have used the convention of dummy indices and omitted the superscript of the unperturbed energy in the denominators, for simplicity.

\section{A.3 Effective matrix for states of interest}

We are interested in ground states with metal sites singly occupied, i.e. $\{|1\rangle,|2\rangle,|10\rangle,|13\rangle\}$ labeled in Figs. A.1 and A.2. By using the four order perturbation mentioned above, 
we finally get

$$
\begin{aligned}
& \mathbf{H}_{\text {eff }}=\left(\begin{array}{ccc}
-\frac{2 t^{2}}{V}+\frac{2 t^{4}}{V^{3}}-\frac{2 t^{4}}{V^{2} U}, & \left(\frac{2 t^{4}}{V^{U}}+\frac{2 t^{4}}{V^{3}}\right) e^{-i 4 \theta} & \\
\left(\frac{2 t^{4}}{V^{U}}+\frac{2 t^{4}}{V^{3}}\right) e^{i 4 \theta}, & -\frac{2 t^{2}}{V}+\frac{2 t^{4}}{V^{3}}-\frac{2 t^{4}}{V^{2} U} & \\
& -\frac{2 t^{2}}{V}+\frac{4 t^{4}}{V^{3}} & \\
& & -\frac{2 t^{2}}{V}+\frac{4 t^{4}}{V^{3}}
\end{array}\right) \\
& =\left(-\frac{2 t^{2}}{V}+\frac{3 t^{4}}{V^{3}}-\frac{t^{4}}{V^{2} U}\right) \mathbf{1}+\left(\frac{4 t^{4}}{V^{2} U}+\frac{4 t^{4}}{V^{3}}\right)\left[\frac{1}{2}\left(S_{1}^{+} S_{2}^{-} e^{-i 4 \theta}+\text { h.c. }\right)+S_{1}^{z} S_{2}^{z}\right],
\end{aligned}
$$

which shows that the neighboring metal ions favor anti-parallel spins.

With the effective Hamiltonian Eq.A.8, we can also get the lowest energies for triplet and singlet states: $-\frac{2 t^{2}}{V}+\frac{4 t^{4}}{V^{3}}-\frac{4 t^{4}}{V^{2} U}-\frac{4 t^{4}}{V^{3}}$ and $-\frac{2 t^{2}}{V}+\frac{4 t^{4}}{V^{3}}$. It is noticeable that the phase factor seems to be as twice as that in double exchange model, but in fact they are the same because the distance between the neighboring metal ions has been doubled due the existence of ligand. 


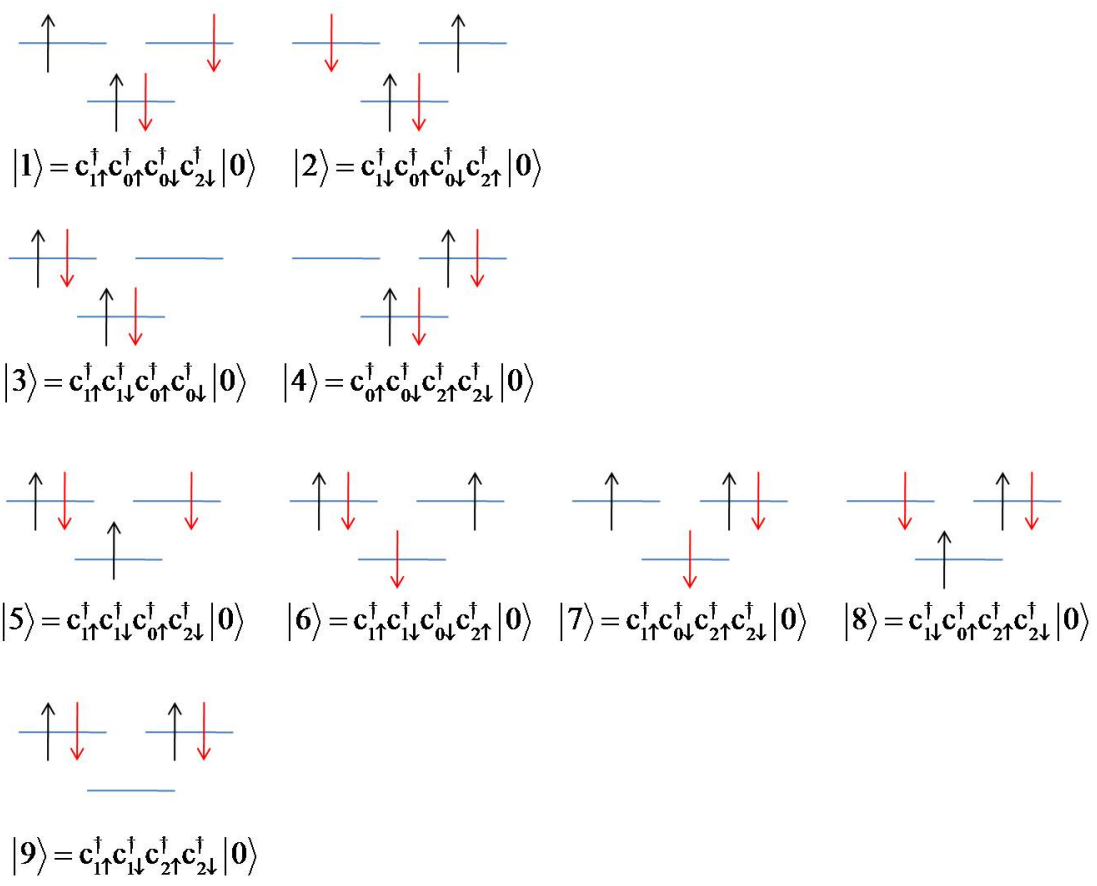

Figure A.1: Subspace $S_{z}^{\text {total }}=0$.
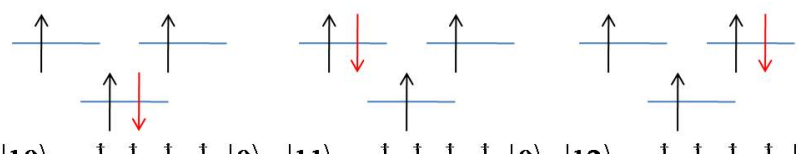

$|10\rangle=c_{1 \uparrow}^{\dagger} c_{0 \uparrow}^{\dagger} c_{0 \downarrow}^{\dagger} c_{2 \uparrow}^{\dagger}|0\rangle$

$|11\rangle=c_{1 \uparrow}^{\dagger} \uparrow_{11}^{\dagger} c_{0 \uparrow}^{\dagger} c_{2 \uparrow}^{\dagger}|0\rangle|12\rangle=c_{1 \uparrow}^{\dagger} c_{0 \uparrow}^{\dagger} c_{2 \uparrow}^{\dagger} c_{2 \downarrow}^{\dagger}|0\rangle$
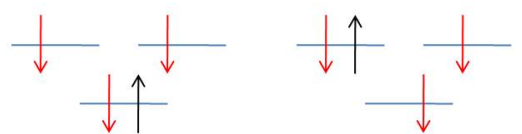

$|13\rangle=c_{1 \downarrow}^{\dagger} c_{0 \uparrow}^{\dagger} c_{0 \downarrow}^{\dagger} c_{2 \downarrow}^{\dagger}|0\rangle|14\rangle=c_{1 \uparrow}^{\dagger} c_{1 \downarrow}^{\dagger} c_{0 \downarrow}^{\dagger} c_{2 \downarrow}^{\dagger}|0\rangle$

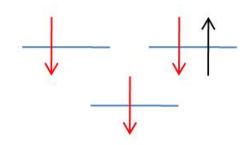

$|15\rangle=c_{1 \downarrow}^{\dagger} c_{0 \downarrow}^{\dagger} c_{2 \downarrow}^{\dagger} c_{2 \uparrow}^{\dagger}|0\rangle$

Figure A.2: Subspace $S_{z}^{\text {total }}= \pm 1$. 


\section{Appendix B}

\section{Magnon-Magnon Interaction}

In this appendix, we study the non-linear properties of spin waves in a ferromagnet. The procedures are summarized as below.

We first expand the magnetization in a power series of canonical variables $a(\mathbf{r})$ and $a^{*}(\mathbf{r})$ (the amplitudes of spin waves) and pass it to k-representation ( $a_{\mathbf{k}}$ and $a_{\mathbf{k}}^{*}$ ) through Fourier transformation. It is a classical analogy to Holstein-Primakoff transformation, but it avoids of difficulties arising from the non-commutativity of operators and the diagonalization of a huge matrix due to the large number of discrete sites in a ferromagnet.

Secondly, we do a canonical transformation (Bogoliubov transformation) to diagonalize the quadratic part of the hamiltonian. The transformation can be obtained by solving the Hamiltonian equations

$$
\dot{a}_{\mathbf{k}}=i \frac{\partial \mathscr{H}}{\partial a_{\mathbf{k}}^{*}}, \quad \dot{a}_{\mathbf{k}}^{*}=-i \frac{\partial \mathscr{H}}{\partial a_{\mathbf{k}}} .
$$


Thirdly, we apply the Bogoliubov transformation to higher order terms in the hamiltonian and get the amplitude of the three- and four- magnon interactions.

\section{B.1 "Holstein-Primakoff" Transformation}

We are interested in a tangentially magnetized ferromagnetic film. The positiondependence of the amplitudes along the thickness direction cannot be described by plane waves, instead, we need to introduce a series of orthogonal functions that satisfy the equation of motion for the magnetization, as well as the electrodynamic and exchange boundary conditions. Suppose the equilibrium magnetization $\mathbf{M}_{0}$ is along $z$-axis and its fluctuation $\mathbf{m}$ is in the $x$ - $y$ plane, then we can expand the magnetization as

$$
\begin{aligned}
& m^{+}(\mathbf{r}, \xi)=a(\mathbf{r}, \xi) \sqrt{1-\frac{\hbar \gamma a(\mathbf{r}, \xi) a^{*}(\mathbf{r}, \xi)}{2 M_{s} \Omega}} \sqrt{\frac{2 \hbar \gamma M_{s}}{\Omega}}, \\
& \simeq \sqrt{\frac{2 \hbar \gamma M_{s}}{V}}\left[\sum_{n \mathbf{k}} a_{n \mathbf{k}} \Phi_{n}(\xi) e^{i \mathbf{k} \cdot \mathbf{r}}\right. \\
& \left.-\frac{\hbar \gamma}{4 M_{s} V} \sum_{n_{1} \mathbf{k}_{1}} \sum_{n_{2} \mathbf{k}_{2}} \sum_{n_{3} \mathbf{k}_{3}} a_{n_{1} \mathbf{k}_{1}} a_{n_{2} \mathbf{k}_{2}} a_{n_{3} \mathbf{k}_{3}}^{*} \Phi_{n_{1}}(\xi) \Phi_{n_{2}}(\xi) \Phi_{n_{3}}(\xi) e^{i\left(\mathbf{k}_{1}+\mathbf{k}_{2}-\mathbf{k}_{3}\right) \cdot \mathbf{r}}\right], \\
& m^{-}(\mathbf{r}, \xi)=a^{*}(\mathbf{r}, \xi) \sqrt{1-\frac{\hbar \gamma a(\mathbf{r}, \xi) a^{*}(\mathbf{r}, \xi)}{2 M_{s} \Omega}} \sqrt{\frac{2 \hbar \gamma M_{s}}{\Omega}}, \\
& \simeq \sqrt{\frac{2 \hbar \gamma M_{s}}{V}}\left[\sum_{n \mathbf{k}} a_{n \mathbf{k}}^{*} \Phi_{n}(\xi) e^{-i \mathbf{k} \cdot \mathbf{r}}\right. \\
& \left.-\frac{\hbar \gamma}{4 M_{s} V} \sum_{n_{1} \mathbf{k}_{1}} \sum_{n_{2} \mathbf{k}_{2}} \sum_{n_{3} \mathbf{k}_{3}} a_{n_{1} \mathbf{k}_{1}}^{*} a_{n_{2} \mathbf{k}_{2}}^{*} a_{n_{3} \mathbf{k}_{3}} \Phi_{n_{1}}(\xi) \Phi_{n_{2}}(\xi) \Phi_{n_{3}}(\xi) e^{-i\left(\mathbf{k}_{1}+\mathbf{k}_{2}-\mathbf{k}_{3}\right) \cdot \mathbf{r}}\right],
\end{aligned}
$$

where $\mathbf{r}$ is the position vector within the film plane, $\xi$ is the coordinate perpendicular to the film, $\gamma=g \mu_{B} / \hbar$ is the gyromagnetic ratio, $M_{s}$ is the saturation magnetization, 
$\Omega$ is the volume of a unit cell, $V$ is the volume of the film, $n$ denotes the different thickness bands and the orthogonal functions $\Phi_{n}(\xi)$ are real and will be given later.

The hamiltonian of the film consists of three terms, the Zeeman term $\left(\mathscr{H}_{H}\right)$, the magnetic dipolar interaction $\left(\mathscr{H}_{d}\right)$ and the exchange interaction $\left(\mathscr{H}_{\text {ex }}\right)$. It is written in the form

$$
\mathscr{H}=\int_{d-\frac{L}{2}}^{d+\frac{L}{2}} d \xi \int d \mathbf{r}\left\{-\mu_{0} M_{z} H_{z}-\frac{1}{2} \mu_{0} \mathbf{M} \cdot \mathbf{h}_{d}+\frac{J}{2}\left[\left(\frac{\partial \mathbf{M}}{\partial \xi}\right)^{2}+\left(\frac{\partial \mathbf{M}}{\partial \mathbf{r}}\right)^{2}\right]\right\}
$$

where $L$ is the thickness of the film, $H_{z}$ is the magnitude of the external field, $\mathbf{h}_{d}$ denotes the dipolar field induced by spin waves and $J$ is the exchange coefficient in units of $\mu_{0} \mathrm{~m}^{2}$.

Substituting

$$
\begin{aligned}
m^{x}(\mathbf{r}, \xi)= & \frac{1}{2} \sqrt{\frac{2 \hbar \gamma M_{s}}{V}}\left[\sum_{n \mathbf{k}}\left(a_{n \mathbf{k}}+a_{n,-\mathbf{k}}^{*}\right) \Phi_{n}(\xi) e^{i \mathbf{k} \cdot \mathbf{r}}\right. \\
& -\frac{\hbar \gamma}{4 M_{s} V} \sum_{n_{1} \mathbf{k}_{1}} \sum_{n_{2} \mathbf{k}_{2}} \sum_{n_{3} \mathbf{k}_{3}}\left(a_{n_{1} \mathbf{k}_{1}} a_{n_{2} \mathbf{k}_{2}} a_{n_{3} \mathbf{k}_{3}}^{*}+a_{n_{1},-\mathbf{k}_{1}}^{*} a_{n_{2},-\mathbf{k}_{2}}^{*} a_{n_{3},-\mathbf{k}_{3}}\right) \\
& \left.\times \Phi_{n_{1}}(\xi) \Phi_{n_{2}}(\xi) \Phi_{n_{3}}(\xi) e^{i\left(\mathbf{k}_{1}+\mathbf{k}_{2}-\mathbf{k}_{3}\right) \cdot \mathbf{r}}\right], \\
m^{y}(\mathbf{r}, \xi)= & \frac{1}{2 i} \sqrt{\frac{2 \hbar \gamma M_{s}}{V}}\left[\sum_{n \mathbf{k}}\left(a_{n \mathbf{k}}-a_{n,-\mathbf{k}}^{*}\right) \Phi_{n}(\xi) e^{i \mathbf{k} \cdot \mathbf{r}}\right. \\
& -\frac{\hbar \gamma}{4 M_{s} V} \sum_{n_{1} \mathbf{k}_{1}} \sum_{n_{2} \mathbf{k}_{2}} \sum_{n_{3} \mathbf{k}_{3}}\left(a_{n_{1} \mathbf{k}_{1}} a_{n_{2} \mathbf{k}_{2}} a_{n_{3} \mathbf{k}_{3}}^{*}-a_{n_{1},-\mathbf{k}_{1}}^{*} a_{n_{2},-\mathbf{k}_{2}}^{*} a_{n_{3},-\mathbf{k}_{3}}\right) \\
& \left.\times \Phi_{n_{1}}(\xi) \Phi_{n_{2}}(\xi) \Phi_{n_{3}}(\xi) e^{i\left(\mathbf{k}_{1}+\mathbf{k}_{2}-\mathbf{k}_{3}\right) \cdot \mathbf{r}}\right], \\
m^{z}(\mathbf{r}, \xi)= & M_{s}-\frac{\hbar \gamma}{V} \sum_{m \mathbf{k}} \sum_{n \mathbf{k}^{\prime}} a_{m \mathbf{k}}^{*} a_{n \mathbf{k}^{\prime}} \Phi_{m}(\xi) \Phi_{n}(\xi) e^{i\left(\mathbf{k}^{\prime}-\mathbf{k}\right) \cdot \mathbf{r}}
\end{aligned}
$$


and keeping only up to the third order of $a_{n \mathbf{k}}\left(a_{n \mathbf{k}}^{*}\right)$, we obtain

$$
\mathscr{H}_{H}=\omega_{H} \sum_{n \mathbf{k}} a_{n \mathbf{k}}^{*} a_{n \mathbf{k}}
$$

with $\omega_{H}=\gamma \mu_{0} H_{z}$ and

$$
\mathscr{H}_{e x}=\frac{J}{\mu_{0}} \omega_{m} \sum_{n \mathbf{k}}\left(q_{n}^{2}+k^{2}\right) a_{m \mathbf{k}}^{*} a_{n \mathbf{k}}
$$

with $\omega_{M}=\gamma \mu_{0} M_{s}$ and $q_{n}=n \pi / L(n=0,1,2 \ldots)$ being the wave number along the thickness direction.

We emphasize here the magnetic dipolar interaction, since it contributes not only to the quadratic terms but also to the cubic terms in the hamiltonian, and introduce two right-hand reference frames, $x-y-z$ and $\zeta-\eta-\xi$, as shown in Fig. B.1. In the $x-y-z$ reference frame, $z$ axis is always along the equilibrium magnetization $\mathbf{M}_{0}$ and $y$ axis lies in the film plane. In the $\zeta-\eta-\xi$ reference frame, $\xi$ axis is perpendicular to the film and $\zeta$ denotes the negative direction of spin-wave wave vector.

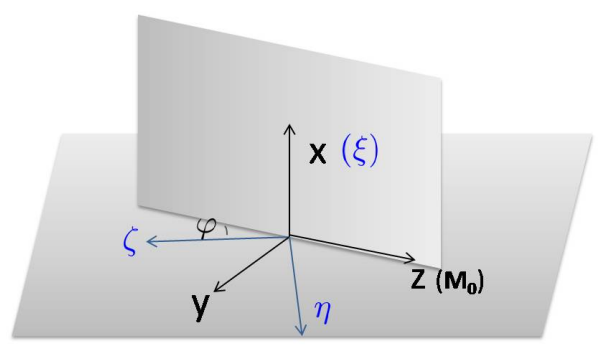

Figure B.1: Two right-hand reference frame: $z$ axis is always along the equilibrium magnetization $\mathbf{M}_{0}$ and $\zeta$ denotes the negative direction of spin-wave wave vector. 


$$
\mathscr{H}_{d}=-\frac{\mu_{0}}{2} \int_{d-\frac{L}{2}}^{d+\frac{L}{2}} d \xi \int d \mathbf{r} \int_{-\frac{L}{2}}^{\frac{L}{2}} d \xi^{\prime} \int d \mathbf{r}^{\prime} m^{\alpha}(\mathbf{r}, \xi) G_{\alpha \beta}\left(\mathbf{r}, \xi ; \mathbf{r}^{\prime}, \xi^{\prime}\right) m^{\beta}\left(\mathbf{r}^{\prime}, \xi^{\prime}\right)
$$

where $\alpha, \beta=x, y, z$. The Fourier transform of $G_{\alpha \beta}\left(\mathbf{r}, \xi ; \mathbf{r}^{\prime}, \xi^{\prime}\right)$ is

$$
G_{\alpha \beta}\left(\mathbf{q}, \xi-\xi^{\prime}\right)=\left(\begin{array}{ccc}
G_{P}-\delta\left(\xi-\xi^{\prime}\right) & i G_{Q} \sin \varphi & -i G_{Q} \cos \varphi \\
i G_{Q} \sin \varphi & -G_{P} \sin ^{2} \varphi & \frac{1}{2} G_{P} \sin 2 \varphi \\
-i G_{Q} \cos \varphi & \frac{1}{2} G_{P} \sin 2 \varphi & -G_{P} \cos ^{2} \varphi
\end{array}\right)
$$

with $G_{P}=\frac{|q|}{2} e^{-|q|\left|\xi-\xi^{\prime}\right|}$ and $G_{Q}=\frac{|q|}{2} e^{-|q|\left|\xi-\xi^{\prime}\right|} \operatorname{sign}\left(\xi-\xi^{\prime}\right)$. Expanding $\mathscr{H}_{d}$ up to the fourth order of $a_{n \mathbf{k}}\left(a_{n \mathbf{k}}^{*}\right)$ and neglecting integrals between different bands yield

$$
\begin{aligned}
\mathscr{H}_{d, \text { inter }}^{(2)}= & -\frac{\omega_{M}}{4} \sum_{n \mathbf{k}} P_{n n} \cos ^{2} \varphi\left(a_{n \mathbf{k}} b_{n \mathbf{k}}^{*}+c . c .\right) \\
& +P_{n n}\left(1+\sin ^{2} \varphi\right)\left(a_{n \mathbf{k}} b_{n,-\mathbf{k}}+\text { c.c. }\right), \\
\mathscr{H}_{d, \text { intra }}^{(2)}= & \omega_{M} \sum_{n \mathbf{k}} a_{n \mathbf{k}} a_{n \mathbf{k}}^{*} \frac{1}{2}\left(1-\cos ^{2} \varphi P_{n n}\right) \\
& +\frac{1}{4}\left\{a_{n \mathbf{k}} a_{n,-\mathbf{k}}\left[1-\left(1+\sin ^{2} \varphi\right) P_{n n}\right]+\text { c.c. }\right\},
\end{aligned}
$$

where

$$
P_{n n}(|\mathbf{k}|)=\int_{d-\frac{L}{2}}^{d+\frac{L}{2}} d \xi \int_{-\frac{L}{2}}^{\frac{L}{2}} d \xi^{\prime} \Phi_{n}(\xi-d) G_{P}\left(|\mathbf{k}|, \xi-\xi^{\prime}\right) \Phi_{n}\left(\xi^{\prime}\right) .
$$

and we have introduced some new canonical variables $b_{n \mathbf{k}}$ and $b_{n \mathbf{k}}^{*}$ to generalize the expression to bilayer system as shown in Fig. B.2. 


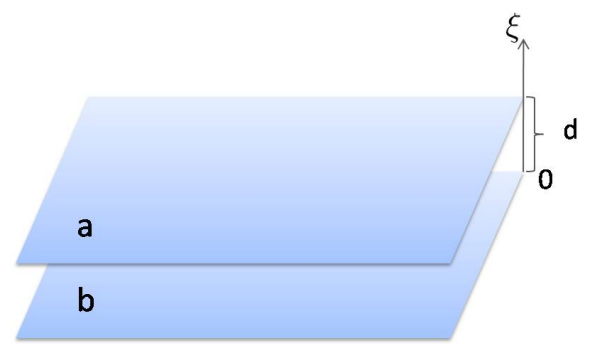

Figure B.2: Schematic picture of ferromagnetic bilayer system, where $d$ is the distance between the two layers. In the top layer the canonical variables are $a_{n \mathbf{k}}$ and $a_{n \mathbf{k}}^{*}$, while in the bottom layer the canonical variables are $b_{n \mathbf{k}}$ and $b_{n \mathbf{k}}^{*}$.

$$
\begin{aligned}
\mathscr{H}_{d, \text { inter }}^{(3)}= & \frac{\sqrt{2 M_{s}}}{4} \mu_{0}\left(\frac{\hbar \gamma}{V}\right)^{\frac{3}{2}} \sum_{n} \sum_{\mathbf{k q}}\left\{a _ { n , - \mathbf { q } } b _ { n , \mathbf { k } } ^ { * } b _ { n , \mathbf { k } + \mathbf { q } } i \left[Q_{1,23}(|\mathbf{q}|) \cos \varphi_{\mathbf{q}}\right.\right. \\
& +\frac{1}{2} P_{1,23}(|\mathbf{q}|) \sin 2 \varphi_{\mathbf{q}}+a_{n, \mathbf{k}}^{*} a_{n, \mathbf{k}+\mathbf{q}} b_{n,-\mathbf{q}} \\
& \left.\times i\left[Q_{23,1}(|\mathbf{q}|) \cos \varphi_{\mathbf{q}}+\frac{1}{2} P_{23,1}(|\mathbf{q}|) \sin 2 \varphi_{\mathbf{q}}\right]+c . c .\right\} \\
\mathscr{H}_{d, \text { inter }}^{(4)}= & \frac{\mu_{0}}{16}\left(\frac{\hbar \gamma}{V}\right)^{2}\left\{\sum_{n} \sum_{\mathbf{k} \mathbf{p}_{1} \mathbf{p}_{2}}\left(a_{n, \mathbf{k}} b_{n, \mathbf{p}_{1}} b_{n, \mathbf{p}_{2}} b_{n, \mathbf{k}+\mathbf{p}_{1}+\mathbf{p}_{2}}^{*}+c . c .\right)\right. \\
& \times\left[P_{1,234}(|\mathbf{k}|)-2 Q_{1,234}(|\mathbf{k}|) \sin \varphi_{\mathbf{k}}+P_{1,234}(|\mathbf{k}|) \sin ^{2} \varphi_{\mathbf{k}}\right] \\
& +\left(a_{n, \mathbf{k}} b_{n, \mathbf{p}_{1}}^{*} b_{n, \mathbf{p}_{2}}^{*} b_{n, \mathbf{p}_{1}+\mathbf{p}_{2}-\mathbf{k}}+c . c .\right) \\
& \times\left[P_{1,234}(|\mathbf{k}|)-2 Q_{1,234}(|\mathbf{k}|) \sin \varphi_{\mathbf{k}}-P_{1,234}(|\mathbf{k}|) \sin ^{2} \varphi_{\mathbf{k}}\right] \\
& +\sum_{n} \sum_{\mathbf{k}_{1} \mathbf{k}_{2} \mathbf{p}}\left(a_{n, \mathbf{k}_{1}} a_{n, \mathbf{k}_{2}} a_{n, \mathbf{k}_{1}+\mathbf{k}_{2}+\mathbf{p}}^{*} b_{n, \mathbf{p}}+c . c .\right) \\
& \times\left[P_{234,1}(|\mathbf{p}|)-2 Q_{234,1}(|\mathbf{p}|) \sin \varphi_{\mathbf{p}}+P_{234,1}(|\mathbf{p}|) \sin ^{2} \varphi_{\mathbf{p}}\right] \\
& \left(a_{n, \mathbf{k}_{1}} a_{n, \mathbf{k}_{2}} a_{n, \mathbf{k}_{1}+\mathbf{k}_{2}-\mathbf{p}}^{*} b_{n, \mathbf{p}}^{*}+c . c .\right) \\
& \left.\times\left[P_{234,1}(|\mathbf{p}|)-2 Q_{234,1}(|\mathbf{p}|) \sin \varphi_{\mathbf{p}}-P_{234,1}(|\mathbf{p}|) \sin ^{2} \varphi_{\mathbf{p}}\right]\right\} \\
& +\frac{\mu_{0}}{2}\left(\frac{\hbar \gamma}{V}\right)^{2} \sum_{n} \sum_{\mathbf{k p q}} a_{n, \mathbf{k}+\mathbf{q}}^{*} a_{n, \mathbf{k}} b_{n, \mathbf{p}-\mathbf{q}}^{*} b_{n, \mathbf{p}} P_{12,34}(|\mathbf{q}|) \cos { }^{2} \varphi_{\mathbf{q}} .
\end{aligned}
$$


where

$$
\begin{aligned}
Q_{1,23}(|\mathbf{q}|) & =\int_{d-\frac{L}{2}}^{d+\frac{L}{2}} d \xi \int_{-\frac{L}{2}}^{\frac{L}{2}} d \xi^{\prime} \Phi_{n}(\xi-d) G_{Q}\left(q, \xi-\xi^{\prime}\right) \Phi_{n}\left(\xi^{\prime}\right) \Phi_{n}\left(\xi^{\prime}\right) \\
P_{1,23}(|\mathbf{q}|) & =\int_{d-\frac{L}{2}}^{d+\frac{L}{2}} d \xi \int_{-\frac{L}{2}}^{\frac{L}{2}} d \xi^{\prime} \Phi_{n}(\xi-d) G_{P}\left(q, \xi-\xi^{\prime}\right) \Phi_{n}\left(\xi^{\prime}\right) \Phi_{n}\left(\xi^{\prime}\right) \\
Q_{23,1}(|\mathbf{q}|) & =\int_{d-\frac{L}{2}}^{d+\frac{L}{2}} d \xi \int_{-\frac{L}{2}}^{\frac{L}{2}} d \xi^{\prime} \Phi_{n}(\xi-d) \Phi_{n}(\xi-d) G_{Q}\left(q, \xi-\xi^{\prime}\right) \Phi_{n}\left(\xi^{\prime}\right), \\
P_{23,1}(|\mathbf{q}|) & =\int_{d-\frac{L}{2}}^{d+\frac{L}{2}} d \xi \int_{-\frac{L}{2}}^{\frac{L}{2}} d \xi^{\prime} \Phi_{n}(\xi-d) \Phi_{n}(\xi-d) G_{P}\left(q, \xi-\xi^{\prime}\right) \Phi_{n}\left(\xi^{\prime}\right),
\end{aligned}
$$

and $\varphi$ is defined in Fig. B.1. Similar definition rules are applied to four-magnon interactions. For example, $P_{1,234}(|\mathbf{q}|)$ has four $\Phi_{n}$ functions in the integral: one with the argument of $\xi-d$; the other three with the argument $\xi^{\prime}$. Suppose the boundaries $\xi= \pm L / 2$ are symmetric, i.e., they are either unpinned or pinned, thus for unpinned boundary conditions

$$
\Phi_{n}(\xi)=\frac{\sqrt{2}}{\sqrt{1+\delta_{o, n}}} \cos \left[q_{n}\left(\xi+\frac{L}{2}\right)\right]
$$

with $q_{n}=\frac{n \pi}{L},(n=0,1,2, \ldots)$; for pinned boundary conditions

$$
\Phi_{n}(\xi)=\sqrt{2} \sin \left[q_{n}\left(\xi+\frac{L}{2}\right)\right]
$$

with $q_{n}=\frac{n \pi}{L},(n=1,2, \ldots)$.

To describe the intralayer three-magnon interaction, one just needs to replace $b$ by $a$, and set $d=0$ in the integrals. Notice that $Q_{1,23}=-Q_{23,1}$ and $P_{1,23}=P_{23,1}$ for 
$d=0$, which simplifies the intralayer three-magnon interaction

$$
\mathscr{H}_{d, \text { intra }}^{(3)}=\frac{\sqrt{2 M_{s}}}{4} \mu_{0}\left(\frac{\hbar \gamma}{V}\right)^{\frac{3}{2}} \sum_{n} \sum_{\mathbf{k q}}\left[a_{n, \mathbf{k}}^{*} a_{n, \mathbf{k}+\mathbf{q}} a_{n,-\mathbf{q}} 2 i P_{1,23}(|\mathbf{q}|) \sin 2 \varphi_{\mathbf{q}}+\text { c.c. }\right]
$$

For very thin films, only the lowest band is excited. We choose the unpinned boundary conditions, under which the lowest band is $n=0$, then $Q_{1,23}=Q_{23,1}=$ $Q_{1,234}=Q_{234,1}=Q(q)$ and $P_{1,23}=P_{23,1}=P_{1,234}=P_{234,1}=P_{12,34}=P(q)$. We get

$$
\begin{aligned}
\mathscr{H}_{d, \text { inter }}^{(3)}= & \frac{\sqrt{2 M_{s}}}{4} \mu_{0}\left(\frac{\hbar \gamma}{V}\right)^{\frac{3}{2}} \sum_{\mathbf{k p}}\left[a_{\mathbf{k}} b_{\mathbf{p}+\mathbf{k}}^{*} b_{\mathbf{p}} i\left(Q(k) \cos \varphi_{\mathbf{k}}+\frac{1}{2} P(k) \sin 2 \varphi_{\mathbf{k}}\right)\right. \\
& +a_{\mathbf{k}+\mathbf{q}}^{*} a_{\mathbf{k}} b_{\mathbf{p}} i\left(Q(p) \cos \varphi_{\mathbf{p}}+\frac{1}{2} P(p) \sin 2 \varphi_{\mathbf{p}}+c . c .\right] \\
\mathscr{H}_{d, \text { intra }}^{(3)}= & \frac{\sqrt{2 M_{s}}}{4} \mu_{0}\left(\frac{\hbar \gamma}{V}\right)^{\frac{3}{2}} \sum_{\mathbf{k q}}\left[a_{\mathbf{k}}^{*} a_{\mathbf{k}+\mathbf{q}} a_{-\mathbf{q}} i P_{00} \sin 2 \varphi_{\mathbf{q}}+c . c .\right] \\
\mathscr{H}_{d, \text { inter }}^{(4)}= & \frac{\mu_{0}}{2}\left(\frac{\hbar \gamma}{V}\right)^{2} \sum_{\mathbf{k p q}} a_{\mathbf{k}+\mathbf{q}}^{*} a_{\mathbf{k}} b_{\mathbf{p}-\mathbf{q}}^{*} b_{\mathbf{p}} P(q) \cos ^{2} \varphi_{\mathbf{q}} .
\end{aligned}
$$

where the subscript $n=0$ has been suppressed, and we have used $\sin 2 \varphi_{\mathbf{k}}=\sin 2 \varphi_{-\mathbf{k}}$. The interlayer three-magnon interaction involves two processes (and their inverse processes) as shown in Fig. 5.3. The terms in the four-magnon interaction Eq. (B.12) fall into two categories - three magnons from one layer interacting with one magnon from the other layer (Fig. 5.4 (a) to (e)) and the Coulomb-interaction-like term (Fig. $5.4(\mathrm{f}))$. Since the amplitude of the former category is only $\frac{1}{8}$ of the later one, I have dropped the first category and will focus on the collision integral due to the Coulomb-like four-magnon interaction in App. B.3. 
Evaluating Eq. (B.10) and (B.14) gives

$$
\begin{aligned}
P_{00}(q) & =1+\frac{e^{-q L}-1}{q L}, \\
Q(q) & =P(q)=\frac{1}{2 q L} e^{-q(d-L)}\left(1-e^{-q L}\right)^{2},
\end{aligned}
$$

with $L$ being the thickness of each layer and $d$ the distance between the two layers. In the long wavelength limit $(q L \gg 1)$ we do not need to do the Bogoliubov transformation and the amplitudes shown above are approximately the amplitudes for three-magnon processes. Detail discussions can be referred to App. B.2.

\section{B.2 Bogoliubov Transformation}

From Eqs. (B.4), (B.5) and (B.9), we can write the quadratic terms of the hamiltonian

$$
\mathscr{H}^{(2)}=\sum_{n \mathbf{k}} A_{n \mathbf{k}} a_{n \mathbf{k}}^{*} a_{n \mathbf{k}}+\frac{1}{2}\left(B_{n \mathbf{k}}^{*} a_{n \mathbf{k}} a_{n,-\mathbf{k}}+\text { c.c. }\right)
$$

where

$$
\begin{aligned}
& A_{n \mathbf{k}}=\omega_{H}+\frac{\omega_{M}}{2}\left(1-\cos ^{2} \varphi P_{n n}\right)+\frac{J}{\mu_{0}}\left(q_{n}^{2}+k^{2}\right), \\
& B_{n \mathbf{k}}^{*}=\frac{\omega_{M}}{2}\left[1-\left(1+\sin ^{2} \varphi\right) P_{n n}\right] .
\end{aligned}
$$

To diagonalize $\mathscr{H}^{(2)}$, we introduce new canonical variables

$$
\begin{aligned}
& c_{n \mathbf{k}}=u_{n \mathbf{k}} a_{n \mathbf{k}}+v_{n \mathbf{k}} a_{n,-\mathbf{k}}^{*} \\
& c_{n \mathbf{k}}^{*}=u_{n \mathbf{k}} a_{n \mathbf{k}}^{*}+v_{n \mathbf{k}}^{*} a_{n,-\mathbf{k}}
\end{aligned}
$$


such that

$$
\begin{aligned}
\mathscr{H}^{(2)}= & \sum_{n \mathbf{k}} \omega_{n \mathbf{k}} c_{n \mathbf{k}}^{*} c_{n \mathbf{k}} \\
= & \sum_{n \mathbf{k}} \omega_{n \mathbf{k}}\left(u_{n \mathbf{k}}^{2} a_{n \mathbf{k}}^{*} a_{n \mathbf{k}}+\left|v_{n \mathbf{k}}\right|^{2} a_{n,-\mathbf{k}}^{*} a_{n,-\mathbf{k}}\right. \\
& \left.+u_{n \mathbf{k}} v_{n \mathbf{k}}^{*} a_{n \mathbf{k}} a_{n,-\mathbf{k}}+u_{n \mathbf{k}} v_{n \mathbf{k}} a_{n \mathbf{k}}^{*} a_{n,-\mathbf{k}}^{*}\right)
\end{aligned}
$$

where we have chosen $u_{n \mathbf{k}}$ to be real and $v_{n \mathbf{k}}$ to be complex. Comparing Eqs. (B.24) and (B.27) and using the constrain, $u_{n \mathbf{k}}^{2}-\left|v_{n \mathbf{k}}\right|^{2}=1$, lead to

$$
\begin{aligned}
\omega_{n \mathbf{k}}^{2} & =A_{n \mathbf{k}}^{2}-\left|B_{n \mathbf{k}}\right|^{2} \\
& =\left[\omega_{H}+\frac{J}{\mu_{0}}\left(q_{n}^{2}+k^{2}\right)+\omega_{M}\left(1-P_{n n}\right)\right]\left[\omega_{H}+\frac{J}{\mu_{0}}\left(q_{n}^{2}+k^{2}\right)+\omega_{M} \sin ^{2} \varphi P_{n(n)}\left(B_{3}, 28\right)\right. \\
u_{n \mathbf{k}} & =\sqrt{\frac{A_{n \mathbf{k}}+\omega_{n \mathbf{k}}}{2 \omega_{n \mathbf{k}}}}, \quad v_{n \mathbf{k}}=\frac{B_{n \mathbf{k}}}{\left|B_{n \mathbf{k}}\right|} \sqrt{\frac{A_{n \mathbf{k}}-\omega_{n \mathbf{k}}}{2 \omega_{n \mathbf{k}}}} .
\end{aligned}
$$

Noting that both $A_{n \mathbf{k}}$ and $B_{n \mathbf{k}}$ are real, $A_{n \mathbf{k}}=A_{n,-\mathbf{k}}$, and $B_{n \mathbf{k}}=B_{n,-\mathbf{k}}$, we obtain the Bogoliubov transformation

$$
\begin{aligned}
a_{n \mathbf{k}} & =u_{n \mathbf{k}} c_{n \mathbf{k}}-v_{n \mathbf{k}} c_{n,-\mathbf{k}}^{*}, \\
a_{n,-\mathbf{k}}^{*} & =-v_{n \mathbf{k}} c_{n \mathbf{k}}+u_{n \mathbf{k}} c_{n,-\mathbf{k}}^{*},
\end{aligned}
$$

where we have used the fact that $u_{n \mathbf{k}}$ and $v_{n \mathbf{k}}$ are real, $u_{n \mathbf{k}}=u_{n,-\mathbf{k}}$ and $v_{n \mathbf{k}}=v_{n,-\mathbf{k}}$. For the bilayer system, we introduce another pair of canonical variables $d_{n \mathbf{k}}$ and $d_{n \mathbf{k}}^{*}$ 
to describe the other layer, that is

$$
\begin{aligned}
b_{n \mathbf{k}} & =u_{n \mathbf{k}}^{\prime} d_{n \mathbf{k}}-v_{n \mathbf{k}}^{\prime} d_{n,-\mathbf{k}}^{*}, \\
b_{n,-\mathbf{k}}^{*} & =-v_{n \mathbf{k}}^{\prime} d_{n \mathbf{k}}+u_{n \mathbf{k}}^{\prime} d_{n,-\mathbf{k}}^{*},
\end{aligned}
$$

where the prime is just a reminder that the dispersion for layer b maybe different from layer a and thus the coefficients for the transformation may also be different.

After doing these transformations and drop the $c_{\mathbf{q}} d_{\mathbf{k}} d_{-\mathbf{k}-\mathbf{q}}$ and $c_{\mathbf{q}}^{*} d_{\mathbf{k}}^{*} d_{-\mathbf{k}-\mathbf{q}}^{*}$ terms, since they break the energy conservation law in the first Born approximation, we achieve at

$$
\begin{aligned}
a_{\mathbf{q}} b_{\mathbf{k}}^{*} b_{\mathbf{k}+\mathbf{q}}+c . c .= & \left(u_{\mathbf{q}} u_{\mathbf{k}}^{\prime} u_{\mathbf{k}+\mathbf{q}}^{\prime}-v_{\mathbf{q}} v_{\mathbf{k}}^{\prime} v_{\mathbf{k}+\mathbf{q}}^{\prime}\right) c_{-\mathbf{q}} d_{\mathbf{k}}^{*} d_{\mathbf{k}+\mathbf{q}} \\
& +\left(-v_{\mathbf{q}} u_{\mathbf{k}}^{\prime} u_{\mathbf{k}+\mathbf{q}}^{\prime}+u_{\mathbf{q}} v_{\mathbf{k}}^{\prime} v_{\mathbf{k}+\mathbf{q}}^{\prime}\right) c_{\mathbf{q}} d_{\mathbf{k}} d_{\mathbf{k}+\mathbf{q}}^{*} \\
& +\left(v_{\mathbf{q}} v_{\mathbf{k}}^{\prime} u_{\mathbf{k}+\mathbf{q}}^{\prime}-u_{\mathbf{q}} u_{\mathbf{k}}^{\prime} v_{\mathbf{k}+\mathbf{q}}^{\prime}\right) c_{\mathbf{q}}^{*} d_{-\mathbf{k}} d_{\mathbf{k}+\mathbf{q}}+c . c .
\end{aligned}
$$

where we have suppressed the band labels, since we are interested in intra-band interaction only. It is worth noting that for the short-wave-length spin waves (i.e. $k L \gg 1$ ), we do not have to do this cumbersome transformation because $A_{\mathbf{k}} \gg B_{\mathbf{k}}$, which yields $u_{\mathrm{k}} \simeq 1$ and $v_{\mathrm{k}} \simeq 0$, and thus the amplitude of three-magnon interaction simply depends on the direction of spin-wave wave vectors as shown in Eqs. (B.20) and (B.21). 


\section{B.3 Rate of Change of Momentum and Thermal Current - Four-Magnon Interaction}

First, we discuss the rate of change of momentum owing to the interlayer Coulomblike four-magnon interaction. It leads to the matrix elements of $B_{\mu \alpha}^{i j}$ with $\alpha=\mu, T$ and $i, j=1,2$. Define the interlayer collision integral $\left(\frac{\partial n_{1}}{\partial t}\right)_{\text {coll } 12}=I(\mathbf{k})$ with

$$
\begin{aligned}
I(\mathbf{k})= & -\sum_{\mathbf{p}} \frac{2 \pi}{\hbar}|W(\mathbf{q})|^{2}\left(n_{1 \mathbf{k}} \bar{n}_{1, \mathbf{k}+\mathbf{q}} n_{2 \mathbf{p}} \bar{n}_{2, \mathbf{p}-\mathbf{q}}-\bar{n}_{1 \mathbf{k}} n_{1, \mathbf{k}+\mathbf{q}} \bar{n}_{2 \mathbf{p}} n_{2, \mathbf{p}-\mathbf{q}}\right) \\
& \times \delta\left(\epsilon_{1 k}+\epsilon_{2 p}-\epsilon_{1, \mathbf{k}+\mathbf{q}}-\epsilon_{2, \mathbf{p}+\mathbf{q}}\right) .
\end{aligned}
$$

Linearize $I(\mathbf{k})$ about $\mathbf{P}_{i}$ and $\mathbf{P}_{T i}$ as defined in Eq. (5.12) and suppose $\beta_{1}=\beta_{2}=\beta$, we have

$$
\begin{aligned}
I(\mathbf{k})= & \beta \frac{4 \pi D}{\hbar^{2}} \sum_{\mathbf{p} \mathbf{q} \omega}|W(\mathbf{q})|^{2}\left\{-\mathbf{q} \cdot\left[\mathbf{P}_{1}+\beta \mathbf{P}_{T 1}\left(D k^{2}+D \frac{q^{2}}{4}+\epsilon_{01}-\mu_{1}\right)\right]\right. \\
& \left.-\mathbf{k} \cdot \mathbf{P}_{T 1} \beta \hbar \omega+\mathbf{q} \cdot\left[\mathbf{P}_{2}+\beta \mathbf{P}_{T 2}\left(D p^{2}+D \frac{q^{2}}{4}+\epsilon_{02}-\mu_{2}\right)\right]+\mathbf{p} \cdot \mathbf{P}_{T 1} \beta \hbar \omega\right\} \\
& \times \delta\left(\epsilon_{1 \mathbf{k}-}-\epsilon_{1 \mathbf{k}+}+\hbar \omega\right) \delta\left(\epsilon_{2 \mathbf{p}+}-\epsilon_{2 \mathbf{p}^{-}}+\hbar \omega\right) .
\end{aligned}
$$

In the calculation of $\sum_{\mathbf{k}} \mathrm{k} I(\mathbf{k})$, we come across the problem of changing the sequence of dot product. Unlike Coulomb interaction, whose amplitude does not depend on the direction of momentum transfer $\mathbf{q}$, the four-magnon interaction strongly depends on the angle between the current density and the equilibrium magnetization. Suppose $\mathbf{P}_{i}$ and $\mathbf{P}_{T i}$ are parallel to $\mathbf{M}_{S}$, we obtain 


$$
\begin{aligned}
B_{\mu \mu}^{11}= & -\beta \frac{4 D}{\pi \hbar^{2}} \frac{5}{16} \sum_{q \omega}|W(q)|^{2} \frac{q^{2}}{2} \chi_{1}^{\prime \prime} \chi_{2}^{\prime \prime} n_{B}(\hbar \omega) n_{B}(-\hbar \omega), \\
B_{\mu T}^{11}= & -\beta \frac{4 D}{\pi \hbar^{2}} \frac{5}{16} \sum_{q \omega}|W(q)|^{2}\left\{\frac{q^{2}}{2} \chi_{1 \epsilon}^{\prime \prime}+\left[\frac{q^{2}}{2}\left(\epsilon_{01}-\mu_{1}\right)+\frac{(\hbar \omega)^{2}}{4 D}\right] \chi_{1}^{\prime \prime}\right\} \\
& \times \chi_{2}^{\prime \prime} n_{B}(\hbar \omega) n_{B}(-\hbar \omega), \\
B_{\mu \mu}^{12}= & -\beta \frac{4 D}{\pi \hbar^{2}} \frac{5}{16} \sum_{q \omega}|W(q)|^{2}\left(-\frac{q^{2}}{2}\right) \chi_{1}^{\prime \prime} \chi_{2}^{\prime \prime} n_{B}(\hbar \omega) n_{B}(-\hbar \omega), \\
B_{\mu T}^{12}= & -\beta \frac{4 D}{\pi \hbar^{2}} \frac{5}{16} \sum_{q \omega}|W(q)|^{2}\left\{-\frac{q^{2}}{2} \chi_{2 \epsilon}^{\prime \prime}+\left[-\frac{q^{2}}{2}\left(\epsilon_{02}-\mu_{2}\right)-\frac{(\hbar \omega)^{2}}{4 D}\right] \chi_{2}^{\prime \prime}\right\} \\
& \times \chi_{1}^{\prime \prime} n_{B}(\hbar \omega) n_{B}(-\hbar \omega),
\end{aligned}
$$

where the summation is over the magnitude of $\mathbf{q}$, and the response functions should be understood as functions of $q$ and $\omega$.

Next, from $\sum_{\mathbf{k}} \mathbf{k} \tilde{\epsilon}_{1 \mathbf{k}} I(\mathbf{k})$ we obtain the rate of change of thermal current. It leads to the matrix elements of $B_{T \alpha}^{i j}$ with $\alpha=\mu, T$ and $i, j=1,2$. 


$$
\begin{aligned}
B_{T \mu}^{11}= & -\beta \frac{4 D}{\pi \hbar^{2}} \frac{5}{16} \sum_{q \omega}|W(q)|^{2}\left\{\frac{q^{2}}{2} \chi_{1 \epsilon}^{\prime \prime}+\left[\frac{q^{2}}{2}\left(\epsilon_{01}-\mu_{1}\right)+\frac{(\hbar \omega)^{2}}{4 D}\right] \chi_{1}^{\prime \prime}\right\} \\
& \times \chi_{2}^{\prime \prime} n_{B}(\hbar \omega) n_{B}(-\hbar \omega), \\
B_{T T}^{11}= & -\beta \frac{4 D}{\pi \hbar^{2}} \frac{5}{16} \sum_{q \omega}|W(q)|^{2}\left\{\frac{(\hbar \omega)^{2}}{2 D}\left[\chi_{1 \epsilon}^{\prime \prime}+\left(\epsilon_{01}-\mu_{1}\right) \chi_{1}^{\prime \prime}\right]\right. \\
& \left.+\frac{q^{2}}{2}\left[\chi_{1 \epsilon \epsilon}^{\prime \prime}+2\left(\epsilon_{01}-\mu_{1}\right) \chi_{1 \epsilon}^{\prime \prime}+\left(\epsilon_{01}-\mu_{1}\right)^{2} \chi_{1}^{\prime \prime}\right]\right\} \chi_{2}^{\prime \prime} n_{B}(\hbar \omega) n_{B}(-\hbar \omega) \\
B_{T \mu}^{12}= & -\beta \frac{4 D}{\pi \hbar^{2}} \frac{5}{16} \sum_{q \omega}|W(q)|^{2}\left\{\left[-\frac{(\hbar \omega)^{2}}{4 D}-\frac{q^{2}}{2}\left(\epsilon_{01}-\mu_{1}\right)\right] \chi_{1}^{\prime \prime}-\frac{q^{2}}{2} \chi_{1 \epsilon}^{\prime \prime}\right\} \\
& \times \chi_{2}^{\prime \prime} n_{B}(\hbar \omega) n_{B}(-\hbar \omega), \\
B_{T T}^{12}= & -\beta \frac{4 D}{\pi \hbar^{2}} \frac{5}{16} \sum_{q \omega}|W(q)|^{2}\left\{-\frac{(\hbar \omega)^{2}}{2 D}\left[\chi_{1 \epsilon}^{\prime \prime}+\left(\epsilon_{01}-\mu_{1}\right) \chi_{1}^{\prime \prime}\right] \chi_{2}^{\prime \prime}\right. \\
& -\frac{q^{2}}{2}\left[\left(\epsilon_{02}-\mu_{2}\right) \chi_{1 \epsilon}^{\prime \prime}+\left(\epsilon_{01}-\mu_{1}\right)\left(\epsilon_{02}-\mu_{2}\right) \chi_{1}^{\prime \prime}\right] \chi_{2}^{\prime \prime} \\
& \left.-\frac{q^{2}}{2}\left[\chi_{1 \epsilon}^{\prime \prime}+\left(\epsilon_{01}-\mu_{1}\right) \chi_{1}^{\prime \prime}\right] \chi_{2 \epsilon}^{\prime \prime}\right\} n_{B}(\hbar \omega) n_{B}(-\hbar \omega) .
\end{aligned}
$$

Some symmetries have been checked. $B_{\mu \mu}^{12}=-B_{\mu \mu}^{11}$ and $B_{\mu T}^{12}=-B_{\mu T}^{11}$ show that the rate of change of momentum is Galilean invariant. Besides, $B_{\mu T}^{11}=B_{T \mu}^{11}$ and $B_{\mu T}^{12}=B_{T \mu}^{12}$ (if the two layers are identical, i.e. $\epsilon_{01}=\epsilon_{02}$ and $\mu_{1}=\mu_{2}$ ) satisfy the Onsager relations. 


\section{Appendix $\mathrm{C}$}

\section{Magnon Response Functions}

The density-density response function

$$
\begin{aligned}
\chi(q, \omega, T) & =\sum_{\mathbf{k}} \frac{n_{k}^{0}-n_{\mathbf{k}+\mathbf{q}}^{0}}{\epsilon_{k}-\epsilon_{\mathbf{k}+\mathbf{q}}+\hbar \omega+i \eta} \\
& =\sum_{\mathbf{k}} \frac{1}{e^{\beta\left(\epsilon_{k}-\mu\right)}-1}\left(\frac{1}{\epsilon_{k}-\epsilon_{\mathbf{k}+\mathbf{q}}+\hbar \omega+i \eta}+\frac{1}{\epsilon_{k}-\epsilon_{\mathbf{k}+\mathbf{q}}-\hbar \omega-i \eta}\right)
\end{aligned}
$$

where $\epsilon_{k}=D k^{2}+\epsilon_{0}$. Taking a similar process as solving the Lindhard function at finite temperature, we substitute

$$
\frac{1}{e^{\beta\left(\epsilon_{k}-\mu\right)}-1}=\int_{0}^{\infty} \frac{\Theta\left(E-D k^{2}\right)}{4 k_{B} T \sinh ^{2}\left(\frac{E+\epsilon_{0}-\mu}{2 k_{B} T}\right)} d E
$$

into Eq. (C.1) and define $E \equiv x^{2}\left(\epsilon_{0}-\mu\right) \equiv D k_{c}^{2}$. With introducing the dimensionless quantities $\nu_{ \pm}=\frac{\bar{\omega}}{2 \bar{q}} \pm \frac{\bar{q}}{2}$, where $\bar{\omega}=\frac{\hbar \omega}{D k_{c}^{2}}$ and $\bar{q}=\frac{q}{k_{c}}$, the imaginary part of the magnon 
response functions can be simplified to be a single integral over $x$,

$$
\begin{aligned}
\chi_{i}^{\prime \prime}(\bar{q}, \bar{\omega}, T)= & -\int_{0}^{\infty} d x \frac{x\left(\epsilon_{0 i}-\mu_{i}\right)}{2 k_{B} T_{i} \sinh ^{2}\left(\left(x^{2}+1\right) \frac{\epsilon_{0 i}-\mu_{i}}{2 k_{B} T_{i}}\right)} \frac{1}{4 \pi D \bar{q}} \\
& \times\left[\Theta\left(x-\left|\nu_{-}\right|\right) \sqrt{x^{2}-\nu_{-}^{2}}-\Theta\left(x-\left|\nu_{+}\right|\right) \sqrt{x^{2}-\nu_{+}^{2}}\right], \quad \text { (C.3) } \\
\chi_{i \epsilon}^{\prime \prime}(\bar{q}, \bar{\omega}, T)= & -\int_{0}^{\infty} d x \frac{x\left(\epsilon_{0 i}-\mu_{i}\right)^{2}}{2 k_{B} T_{i} \sinh ^{2}\left(\left(x^{2}+1\right) \frac{\epsilon_{0 i}-\mu_{i}}{2 k_{B} T_{i}}\right)} \frac{1}{4 \pi D \bar{q}} \\
& \times\left[\Theta\left(x-\left|\nu_{-}\right|\right) \sqrt{x^{2}-\nu_{-}^{2}}\left(\frac{\bar{q}^{2}}{6}+\frac{x^{2}}{3}+\frac{\bar{\omega}}{6}+\frac{\bar{\omega}^{2}}{6 \bar{q}}\right)\right. \\
\chi_{i \epsilon \epsilon}^{\prime \prime}(\bar{q}, \bar{\omega}, T)= & -\int_{0}^{\infty} d x \frac{x^{2}\left(\epsilon_{0 i}-\mu_{i}\right)^{3}}{2 k_{B} T_{i} \sinh ^{2}\left(\left(x^{2}+1\right) \frac{\epsilon_{0 i}-\mu_{i}}{2 k_{B} T_{i}}\right)} \frac{1}{4 \pi D \bar{q}}\left[\Theta\left(x-\left|\nu_{+}\right|\right) \sqrt{x^{2}-\nu_{+}^{2}}\left(\frac{\bar{q}^{2}}{6}+\frac{x^{2}}{3}-\frac{\bar{\omega}}{6}+\frac{\bar{\omega}^{2}}{6 \bar{q}}\right)\right], \\
& \times\left(\frac{\bar{q}^{4}}{30}+\frac{\bar{q}^{2} x^{2}}{15}+\frac{x^{4}}{5}+\frac{\bar{q}^{2} \bar{\omega}}{30}+\frac{x^{2} \bar{\omega}}{5}+\frac{7}{60} \bar{\omega}^{2}+\frac{x^{2} \bar{\omega}^{2}}{15 \bar{q}^{2}}+\frac{\bar{\omega}^{3}}{30 \bar{q}^{2}}+\frac{\bar{\omega}^{4}}{30 \bar{q}^{4}}\right) \\
& -\Theta\left(x-\left|\nu_{+}\right|\right) \sqrt{x^{2}-\nu_{+}^{2}} \\
& \times\left(\frac{\bar{q}^{4}}{30}+\frac{\bar{q}^{2} x^{2}}{15}+\frac{x^{4}}{5}-\frac{\bar{q}^{2} \bar{\omega}}{30}-\frac{x^{2} \bar{\omega}}{5}+\frac{7}{60} \bar{\omega}^{2}+\frac{x^{2} \bar{\omega}^{2}}{15 \bar{q}^{2}}-\frac{\bar{\omega}^{3}}{30 \bar{q}^{2}}+\frac{\left.\bar{\omega}^{4}(C) .5 .\right)}{\left.30 \bar{q}^{4}\right)}\right.
\end{aligned}
$$




\section{Bibliography}

[1] A. Khitun, M. Bao, and K. L Wang. Magnonic logic circuits. Journal of Physics D: Applied Physics, 43:264005, 2010.

[2] A A Serga, A V Chumak, and B Hillebrands. YIG magnonics. Journal of Physics D: Applied Physics, 43(26):264002, July 2010.

[3] Y. Kajiwara, K. Harii, S. Takahashi, J. Ohe, K. Uchida, M. Mizuguchi, H. Umezawa, H. Kawai, K. Ando, K. Takanashi, S. Maekawa, and E. Saitoh. Transmission of electrical signals by spin-wave interconversion in a magnetic insulator. Nature, 464(7286):262-266, March 2010.

[4] C. W. Sandweg, Y. Kajiwara, A. V. Chumak, A. A. Serga, V. I. Vasyuchka, M. B. Jungfleisch, E. Saitoh, and B. Hillebrands. Spin pumping by parametrically excited exchange magnons. Physical Review Letters, 106(21):216601, May 2011.

[5] M. P Kostylev, A. A Serga, T. Schneider, B. Leven, and B. Hillebrands. Spinwave logical gates. Applied Physics Letters, 87(15):153501-153501-3, October 2005. 
[6] G. T. Rado, C. Vittoria, J. M. Ferrari, and J. P. Remeika. Linear electric field shift of a ferromagnetic resonance: Lithium ferrite. Physical Review Letters, 41(18):1253-1255, October 1978.

[7] Hosho Katsura, Naoto Nagaosa, and Alexander V. Balatsky. Spin current and magnetoelectric effect in noncollinear magnets. Physical Review Letters, 95(5), July 2005.

[8] Taka-hisa Arima. Ferroelectricity induced by Proper-Screw type magnetic order. Journal of the Physical Society of Japan, 76(7):073702, June 2007.

[9] P. Rovillain, R. de Sousa, Y. Gallais, A. Sacuto, M. A. Masson, D. Colson, A. Forget, M. Bibes, A. Barthlmy, and M. Cazayous. Electric-field control of spin waves at room temperature in multiferroic BiFeO3. Nature Materials, 9(12):975979, November 2010.

[10] Pio Baettig and Tamio Oguchi. Why are garnets not ferroelectric? a theoretical investigation of Y3Fe5O12. Chem. Mater., 20(24):7545-7550, 2008.

[11] Zhiliang Cao, Xueping Yu, and Rushan Han. Quantum phase and persistent magnetic moment current and Aharonov-Casher effect in a $s=1 / 2$ mesoscopic ferromagnetic ring. Physical Review B, 56(9):5077-5079, 1997.

[12] A. Slachter, F. L. Bakker, J-P. Adam, and B. J. van Wees. Thermally driven spin injection from a ferromagnet into a non-magnetic metal. Nature Physics, 6(11):879-882, September 2010. 
[13] J. Flipse, F. L. Bakker, A. Slachter, F. K. Dejene, and B. J. van Wees. Direct observation of the spin-dependent peltier effect. Nature Nanotechnology, 7(3):166-168, February 2012.

[14] Gerrit E.W. Bauer, Allan H. MacDonald, and Sadamichi Maekawa. Spin caloritronics. Solid State Communications, 150(11C12):459-460, March 2010.

[15] K. Uchida, S. Takahashi, K. Harii, J. Ieda, W. Koshibae, K. Ando, S. Maekawa, and E. Saitoh. Observation of the spin seebeck effect. Nature, 455(7214):778-781, October 2008.

[16] C. M. Jaworski, J. Yang, S. Mack, D. D. Awschalom, J. P. Heremans, and R. C. Myers. Observation of the spin-Seebeck effect in a ferromagnetic semiconductor. Nat Mater, 9(11):898-903, November 2010.

[17] K. Uchida, J. Xiao, H. Adachi, J. Ohe, S. Takahashi, J. Ieda, T. Ota, Y. Kajiwara, H. Umezawa, H. Kawai, G. E. W. Bauer, S. Maekawa, and E. Saitoh. Spin seebeck insulator. Nat Mater, 9(11):894-897, November 2010.

[18] V. Cherepanov, I. Kolokolov, and V. L'vov. The saga of YIG: spectra, thermodynamics, interaction and relaxation of magnons in a complex magnet. Physics reports, 229(3):81C144, 1993.

[19] A. Lehmann-Szweykowska, A. Szamer, R. J. Wojciechowski, R. Micnas, and T. Lulek. A microscopic model of oxygen vacancies in ca-doped YIG. In Journal of Physics: Conference Series, volume 30, page 278, 2006.

[20] T?ru Moriya. Anisotropic superexchange interaction and weak ferromagnetism. Physical Review, 120(1):91-98, October 1960. 
[21] B A Kalinikos and A N Slavin. Theory of dipole-exchange spin wave spectrum for ferromagnetic films with mixed exchange boundary conditions. Journal of Physics C: Solid State Physics, 19(35):7013-7033, December 1986.

[22] Evgenij Mihajlovic Lifsic, Lev Petrovich Pitaevskii, John Bradbury Sykes, and M. J Kearsley. Statistical physics. Part 2, Theory of the condensed state. Butterworth-Heinemann, Oxford [etc.], 2006.

[23] V. Dugaev, P. Bruno, B. Canals, and C. Lacroix. Berry phase of magnons in textured ferromagnets. Physical Review B, 72(2), July 2005.

[24] Hans-Benjamin Braun and Daniel Loss. Berrys phase and quantum dynamics of ferromagnetic solitons. Physical Review B, 53(6):3237-3255, February 1996.

[25] M. Bailyn. Maximum variational principle for conduction problems in a magnetic field, and the theory of magnon drag. Physical Review, 126(6):2040-2054, June 1962.

[26] F. J. Blatt, D. J. Flood, V. Rowe, P. A. Schroeder, and J. E. Cox. Magnon-drag thermopower in iron. Physical Review Letters, 18(11):395-396, March 1967.

[27] G. N. Grannemann and L. Berger. Magnon-drag peltier effect in a ni-cu alloy. Physical Review B, 13(5):2072-2079, March 1976.

[28] Byung Hoon Kim, Jun Sung Kim, Tae Hoi Park, Dea Su Lee, and Yung Woo Park. Magnon drag effect as the dominant contribution to the thermopower in Bi0.5-xLaxSr0.5MnO3 $(0.1 \leq \mathrm{x} \leq 0.4)$. Journal of Applied Physics, 103(11):113717-113717-4, June 2008. 
[29] Marius V. Costache, German Bridoux, Ingmar Neumann, and Sergio O. Valenzuela. Magnon-drag thermopile. Nat Mater, advance online publication, December 2011.

[30] Steven S.-L. Zhang and Shufeng Zhang. Magnon mediated electric current drag across a ferromagnetic insulator layer. Physical Review Letters, 109(9):096603, August 2012.

[31] T. Holstein and H. Primakoff. Field dependence of the intrinsic domain magnetization of a ferromagnet. Physical Review, 58(12):1098-1113, December 1940.

[32] S. O. Demokritov, V. E. Demidov, O. Dzyapko, G. A. Melkov, A. A. Serga, B. Hillebrands, and A. N. Slavin. Bose-einstein condensation of quasi-equilibrium magnons at room temperature under pumping. Nature, 443(7110):430-433, 2006.

[33] R. A. Duine and H. T. C. Stoof. Spin drag in noncondensed bose gases. Physical Review Letters, 103(17):170401, October 2009.

[34] Roland Winkler. Spin-orbit coupling effects in two-dimensional electron and hole systems. Springer, Berlin; New York, 2003. 


\section{VITA}

Tianyu Liu received her degree of Bachelor of Science in Physics from Tianjin University, China, in July, 2006. Then she continued her study on phonon transportation through nanostructures as a graduate student at the same university, and got her Master degree in July, 2008. Since Fall 2008, Tianyu started pursuing her doctoral degree, supervised by Prof. Vignale and received her PhD degree in Physics at the University of Missouri-Columbia, in December, 2013. 